DOE/ID-10054(98)

July 1999

U.S. Department of Energy Idaho Operations Office

\title{
Radioactive Waste Information for 1998 and Record-To-Date
}




\section{DISCLAIMER}

This report was prepared as an account of work sponsored by an agency of the United States Government. Neither the United States Government nor any agency thereof, nor any of their employees, make any warranty, express or implied, or assumes any legal liability or responsibility for the accuracy, completeness, or usefulness of any information, apparatus, product, or process disclosed, or represents that its use would not infringe privately owned rights. Reference herein to any specific commercial product, process, or service by trade name, trademark, manufacturer, or otherwise does not necessarily constitute or imply its endorsement, recommendation, or favoring by the United States Government or any agency thereof. The views and opinions of authors expressed herein do not necessarily state or reflect those of the United States Government or any agency thereof. 


\section{DISCLAIMER}

Portions of this document may be illegible in electronic image products. Images are produced from the best available original document. 


\title{
Radioactive Waste Information For 1998 And Record-To-Date
}

\author{
D.L. French \\ R. E. Tallman \\ K.A. Taylor
}

Waste Generator Services

Published July 1999

Idaho National Engineering and Environmental Laboratory

Lockheed Martin Idaho Technologies

Idaho Falls, Idaho 83415 



\section{ABSTRACT}

This document presents detailed data, bar graphs, and pie charts on volume, radioactivity, isotopic identity, origin, and status of radioactive waste for calendar year 1998. It also summarizes the radioactive waste data records compiled from 1952 to present for the Idaho National Engineering and Environmental Laboratory (INEEL). The data presented are from the INEEL Integrated Waste Information System. 


\section{EXECUTIVE SUMMARY}

This document, Radioactive Waste Information for 1998 and Record-To-Date, contains computerized radioactive waste data records from the Idaho National Engineering and Environmental Laboratory (INEEL). Data are compiled from information supplied by the U.S. Department of Energy (DOE) contractors. This report provides data on airborne and liquid radioactive effluents and solid radioactive waste that is stored, disposed, or treated at the INEEL for volume reduction. This report provides summarized data for the years 1952 through 1997 and detailed data for the calendar year 1998.

The Environmental Database Integration project is on going with migration to the INEEL Containerized Waste Tracking System (IWTS) version 3 accomplished in May 1999.

As part of the original June 1997 conversion to IWTS extensive verification of older containers was performed. This verification has continued and additional duplicate containers have been identified in the Waste Experimental Reduction Facility (WERF) inventory. When identified, the containers have been archived from the old Radioactive Waste Management Information System (RWMIS), thus eliminating double counting. Task History dates on older containers continue to affect the reporting year. As the backlog is worked off this will be eliminated. A disposal location conversion deficiency has also been found. This affected TRA for 1997 and WERF for 1995. This verification is a continuing process.

Verification of waste profile information is an ongoing activity. As discrepancies are identified and recharacterization of the waste is completed, history totals on the Record-to-Date Summary report TT22EB01 will be updated.

Hanford waste reported on SS22MB01 is waste pending treatment at WERF. After treatment the waste will be shipped off-site for disposal.
Idaho State University (ISU) waste reported on SS22PB01 is INEEL generated waste retumed after Treatability Studies have been performed.

Data used in producing this annual report is from the Integrated Waste Tracking System data repository using the data download of May 10, 1998.

Airborne and liquid waste was released to the environment through engineered release points identified on Table 1 in the Engineered Release Points section. Monitoring and effluent sampling systems are an integral part of each engineered release point. Releases to the environment may also occur at locations other than normal release points.

In 1998, 5,921 curies of airborne radioactivity were released, of which 99.9 percent were noble gases. In 1997 airborne activity release was 5,327 curies and in 1996 it was 2,904 curies.

A total of $1.972 \mathrm{E}+09$ liters containing 78 curies of liquid radioactive waste was released to the INEEL environment during 1998. Ninety-six percent of the radioactivity was tritium. The volume of radioactive liquid discharged as waste in 1998 was 16 percent less than that discharged in 1997 and 12 percent less than that discharged in 1996.

During 1998, a total of $4,224 \mathrm{~m}^{3}$ of containerized radioactive waste containing 5,738 curies was shipped to the Radioactive Waste Management Complex (RWMC) for disposal. During $1997,1,564 \mathrm{~m}^{3}$ containing 15,972 curies was shipped for disposal. During $1996,677 \mathrm{~m}^{3}$ containing 14,445 curies was shipped for disposal.

The majority of the nuclides disposed in solid waste at RWMC in 1998 consisted of Ni-63, Fe-55, Co-58, Co-60. These nuclides accounted for $83 \%$ of the total curie activity. 
Radioactive effluent discharges at the INEEL in 1998 met all applicable DOE requirements (DOE 5400.5, "Radiation Protection of the Public and Environment," and 5820.2, "Radioactive Waste Management") and State of Idaho regulations and standards.

The impact of this waste on the environment, both onsite and offsite, is constantly monitored by the INEEL Site Environmental Surveillance Program. The results of this program for 1998 are published in the annual report, The Idaho National Engineering Laboratory Site Environmental Report for Calendar Year 1998, DOE-/ID-12082(98).

vi 


\section{CONTENTS}

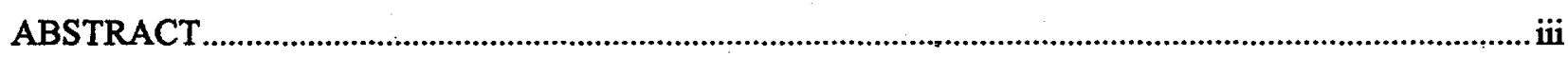

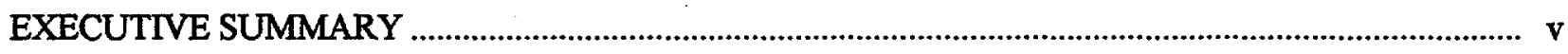

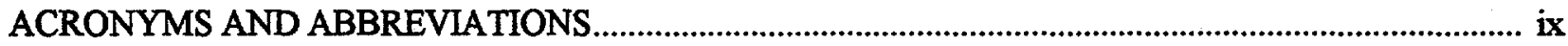

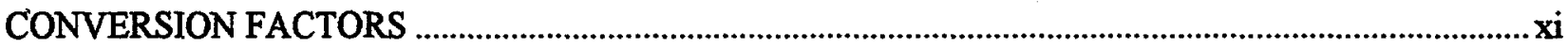

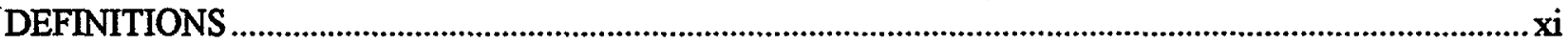

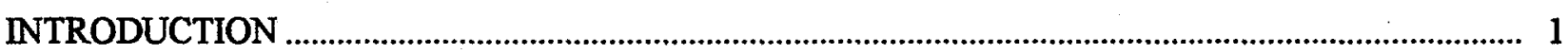

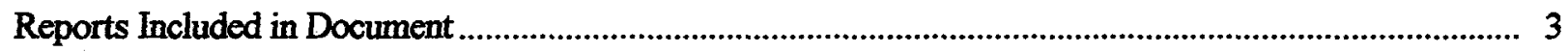

\section{TAB SECTIONS}

INEEL Record-to-Date Summary

INEEL Record-to-Date Discharges and Solid Waste

INEEL 1998 Year-to-Date Summary

Nuclide Summary in Curies for Airborne, Liquid, and Solid Waste

Argonne National Laboratory-West 1998 Graphics

Central Facilities Area 1998 Graphics

Idaho Nuclear Technology and Engineering Center 1998 Graphics

Decontamination and Decommissioning 1998 Graphics

Naval Reactor Facility 1998 Graphics

Power Burst Facility 1998 Graphics

Special Manufacturing Capability 1998 Graphics

Test Area North 1998 Graphics

Test Reactor Area 1998 Graphics

Waste Area Groups

Waste Experimental Reduction Facility 1998 Graphics

Waste Reduction Operation Complex

Engineered Release Points 


\section{ACRONYMS AND ABBREVIATIONS}

A1W Large ship reactor at Naval Reactors Facility

ANL-E Argonne National Laboratory-East

ANL-W Argonne National Laboratory-West

ARA Auxiliary Reactor Area

ATR Advanced Test Reactor

BCL Battelle Columbus Laboratories

BEN Bendix - Grand Junction, Colorado

BET Bettis Atomic Power Laboratory

BNL Brookhaven National Laboratory

B\&W Babcock \& Wilcox

CEG Combustion Engineering General

Atomics

CF Central Facilities

CG Concentration Guide (also referred to as DCG)

CPP Chemical Processing Plant (previous name for INTEC)

CTF Containment Test Facility (LOFT became CTF on 10/01/86)

CY calendar year

D\&D decontamination and decommissioning

DCG Derived Concentration Guide

DOE U.S. Department of Energy

DOE-ID U.S. Department of Energy, Idaho Operations Office

EBR ANLW Storage Area

EBR-II Experimental Breeder Reactor II

ECF Expended Core Facility

ETR Engineering Test Reactor

FAST Fluorinel and Storage Facility

FCF Fuel Cycle Facility

FMF Fuel Manufacturing Facility

JCH J.C. Haynes/Wright Patterson Air

Force Base
HFEF Hot Fuels Examination Facility

ILTSF Intermediate-Level Transuranic

Storage Facility (also referred to as

IIT)

INEEL Idaho National Engineering and Environmental Laboratory

INTEC Idaho Nuclear Technology and Engineering Center

ISU Idaho State University

IWTS Integrated Waste Tracking System

KAPCT Knolls Atomic Power Laboratory Connecticut

KAPNY Knolls Atomic Power Laboratory New York

LMITCO Lockheed Martin Idaho Technologies Company

L\&O Lab and Office Building

LOF Loss-of-Fluid Test

LOFT Loss-of-Fluid Test

LOS Los Alamos National Laboratory

MDL Monsanto Dayton Laboratory

MRC Monsanto Research Corporation Mound Laboratory

MTR Materials Test Reactor

NNS Norfolk Naval Ship Yard

NRF Naval Reactors Facility

NWCF New Waste Calcining Facility

PBF Power Burst Facility

PER Special Power Excursion Reactor Test (also referred to as SPERT)

PHNSY Pearl Harbor Naval Shipyard

PREPP Process Reduction Experimental Pilot Plant

PSNSY Pudget Sound Naval Shipyard

R\&D research and development 
RESL Radiological and Environmental

Sciences Laboratory

RFO Rocky Flats Office (Kaiser/Hill Rocky

Flats, Colorado)

RLWTF Radioactive Liquid Waste Treatment

RWDS Radioactive Waste Disposal System

RWMC Radioactive Waste Management

Complex (also referred to as WMF)

RWMIS Radioactive Waste Management

Information System

S1W submarine prototype at NRF

S5G submarine prototype at NRF

SCND Special Case Non-defense Waste

SEG Scientific Ecology Group

SL-1 Stationary Low Power Reactor No. 1

SMC Special Manufacturing Capability

SPF Sodium Process Facility

SWEPP Stored Waste Examination Pilot Plant
TAN Test Area North

TRA Test Reactor Area

TRIPS Transuranic Reporting Inventory and Processing System

TSA Transuranic Storage Area

TSF Test Support Facility

WAG Waste Area Groups

WAG1 Test Area North

WAG3 Idaho Nuclear Technology and Engineering Center

WAG7 Waste Management Facility

WERF Waste Experimental Reduction Facility (also referred to as WER)

WIPP Waste Isolation Pilot Project

WMF Waste Management Facility (also referred to as RWMC)

WROC Waste Reduction Operations Complex ZPPR Zero Power Plutonium Reactor 


\section{CONVERSION FACTORS}

\begin{tabular}{llr} 
To Convert & \multicolumn{1}{c}{ Into } & Multiply By \\
\cline { 3 - 3 } Cubic yards & Cubic meters & 0.7646 \\
Cubic meters & Cubic feet & 35.3140 \\
Liters & Gallons (U.S. liquid) & 0.2642 \\
Kilograms & Pounds & 2.2046
\end{tabular}

\section{DEFINITIONS}

Curie

A unit of radioactivity, defined as that quantity of any radioactive nuclide that has 3.7E+10 disintegrations per second (abbreviated $\mathrm{Ci}$ ).

Exponential notation Numbers in this document are expressed as either whole numbers or in exponential notation form. For example: $1,000,000=1 \times 10^{6}=1 E+06$. 


\section{RADIOACTIVE WASTE MANAGEMENT INFORMATION FOR 1998 AND RECORD-TO-DATE}

\section{INTRODUCTION}

This document summarizes radioactive waste data records for the Idaho National Engineering and Environmental Laboratory (INEEL) compiled since 1952, and provides detailed data for calendar year (CY) 1998. A computerized radioactive waste tracking system has been used at the INEEL since January 1971.

Several improvements to the data base system and data reporting have taken place since data collection was computerized. These improvements include:

- In 1982, the data base language was converted from COBOL to NOMAD, a fourth generation data base language. NOMAD had many statistical analysis features that were applied to the data, and allowed for modifying data reporting requirements with minimal impact on the data base.

- In January 1986 , solid waste data reporting requirements changed from a per-shipment basis to a per-container basis. A shipment could consist of 1 to 70 containers. By changing to a per-container basis, solid waste location on the INEEL is tracked more accurately. It is now possible to identify each container and to determine its waste classification.

- In February 1988, waste generators were given the ability to provide the analytical uncertainty, at one sigma, for the reported nuclides of air and liquid effluent releases.

- In May 1996, the process to convert the data from NOMAD to the Oracle language identified one improper location coding. The correction of this coding caused 10 cubic meters and 82 curies, not previously reported, to now be reported for WMC 1987 TSA total.

- In June 1996 the conversion from NOMAD to the ORACLE platform was completed. As a result of this platform change the precision of waste calculation results has been improved. Using the NOMAD code with a value retrieved for use in calculations the value was truncated. For example if the original number was $2,440,132,560$ it would be truncated for calculations to $2.440 \mathrm{E}+06$ resulting in a loss of 132,560 . The greater the original number, the greater the precision loss. With the ORACLE platform for calculations the numbers are rounded rather than truncated. This change will show up as differences between the older NOMAD reports and the current ORACLE calculation method.

- In June 1997 the Environmental Database Integration project was initiated to bring together waste tracking systems across the NNEEL into one reporting system. Historical information from the Radioactive Waste Management Information System (RWMIS) was loaded into a data repository. All previous reporting capabilities are available in the Integrated Waste Tracking System (IWTS) along with additional detailed information for tracking containerized waste that was previous unavailable.

U.S. Department of Energy (DOE) contractors routinely report information on airborne and liquid radioactive effluents and solid radioactive waste that is stored, disposed, and sent to the INEEL for volume reduction. Types of information include 
volume, radioactivity, isotopic identity, and origin. This system serves as the official repository for this data and provides reports for all types of radioactive effluents and waste disposed, sent for volume reduction, or stored at the INEEL.

Graphics were added to the report in 1987 to improve the data presentation. The area-specific solid low-level waste graphs in this report depict waste in five categories:

1. Direct Disposal-waste that is sent directly to the Radioactive Waste Management Complex for disposal with no volume reduction efforts applied.

2. Compaction-Compatible waste that is sent to the Waste Experimental Reduction Facility (WERF) to be compacted.

3. Metal Sizing-Metallic waste that is sent to WERF for volume reduction.

4. Incineration-Combustible waste sent to WERF for incineration.

5. Waste shipped to off-site facilities for volume reduction or disposal.

In 1990, additional reports and graphics were added to better represent previously summarized data. These reports and graphics included a detailed list of generators who ship waste to WERF and details on stored waste by generator.

The waste tracking system is continually undergoing review and enhancement. Comments on the system are encouraged. This report is updated annually to incorporate waste management data for the current year and to reflect changes from previous annual reports. These changes are made to more accurately reflect the current status of waste operations at the INEEL.
Annual and special waste reports are useful to various levels of management in appraising their radioactive waste programs. Annual reports provide a summary by type and producer of waste. The volume, weight, and curie content in all reports are expressed in exponential notation.

A comparison of the annual mean average released nuclides concentrations and the Derived Concentrations Guide (DCG) limits are included in the detailed tracking system. The concentration of liquid releases is compared directly to the reference DCG limits for drinking water. It must be noted that the DCG liquid release limits are for protecting the public from ingesting radiationcontaminated water. INEEL liquid releases flow to areas inaccessible to the general public. The calculated concentration of airborne releases as dispersed to the INEEL boundary is compared to the reference airborne DCG limits. The reference DCG values are given in DOE Order 5400.5, "Radiation Protection of the Public and the Environment," February 1990. The right-hand column of many reports indicate total and average values. Total values include the total content in curies for each radionuclide released during the year. Average values are the annual mean concentrations of radionuclides.

The tracking and reporting system provides readily available information that permits ongoing evaluation of INEEL waste management activities, including compliance with DOE regulations and those of other Federal and State agencies. The database continues to be a valuable asset to the overall management effort at the INEEL and continues to alert the DOE Idaho Operations Office (DOE-ID) of trends and potential problem areas. 


\section{Reports Included in Document}

Six report categories are included in this document. Brief descriptions of each are below.

INEEL Record-to-Date Summary-This report summarizes the volume and curies of all effluents and solid waste on the INEEL by CY for the period 1989 to 1998 and a cumulative total for 1952 to 1988.

\section{INEEL Record-to-Date Discharges and} Solid Waste-These reports summarize the volume and curies of all discharges and solid waste on the INEEL by discharge/waste type for each area generating waste by CY for the period 1989 to 1998 and a cumulative total for 1952 to 1988.

INEEL 1998 Year-to-Date Summary-This report summarizes, by facility, the amounts of radioactivity generated at INEEL facilities or at offsite facilities and shipped to the INEEL. Liquid and airborne waste that is released is identified, and quantities of solid wastes stored, disposed, or received for reduction are given. The volume of the media containing the radioactivity is also included.

Nuclide Summary in Curies for Airborme, Liquid, and Solid Waste-These reports summarize the annual curie values, by nuclide, for each area reporting effluents or solid waste on the INEEL.

Area 1998 Graphics-These graphics include volume and curie monthly data for airborne, liquid, and solid waste generated by INEEL areas and for non-INEEL areas shipping solid waste to the INEEL.

Engineered Release Points-These tables identify the area and type of discharge of the airborne and liquid waste released to the environment. 
INEEL Record-to-Date Summary INEEL-3

INEEL Record-to-Date Summary Bar Graphs INEEL-4 



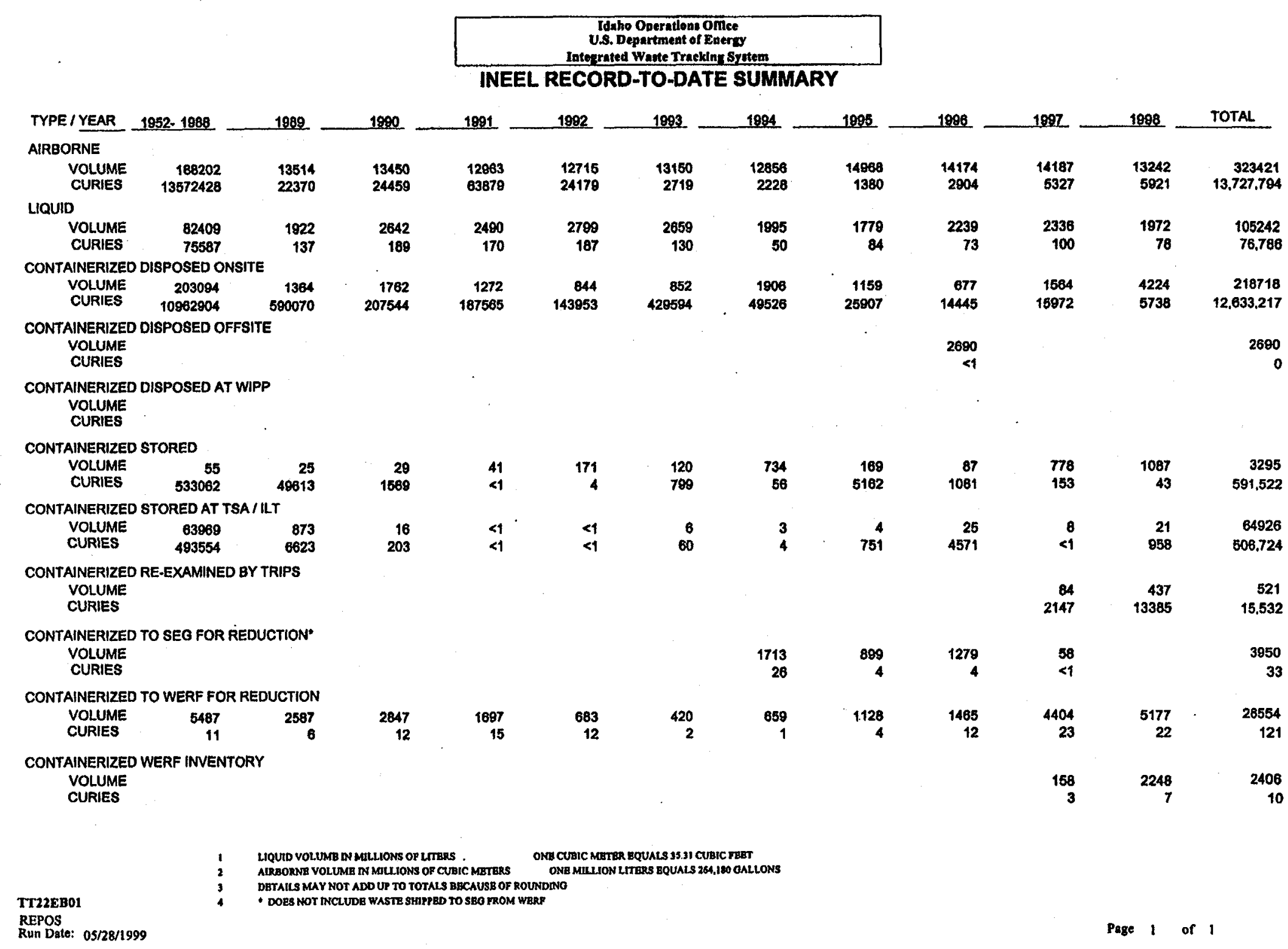



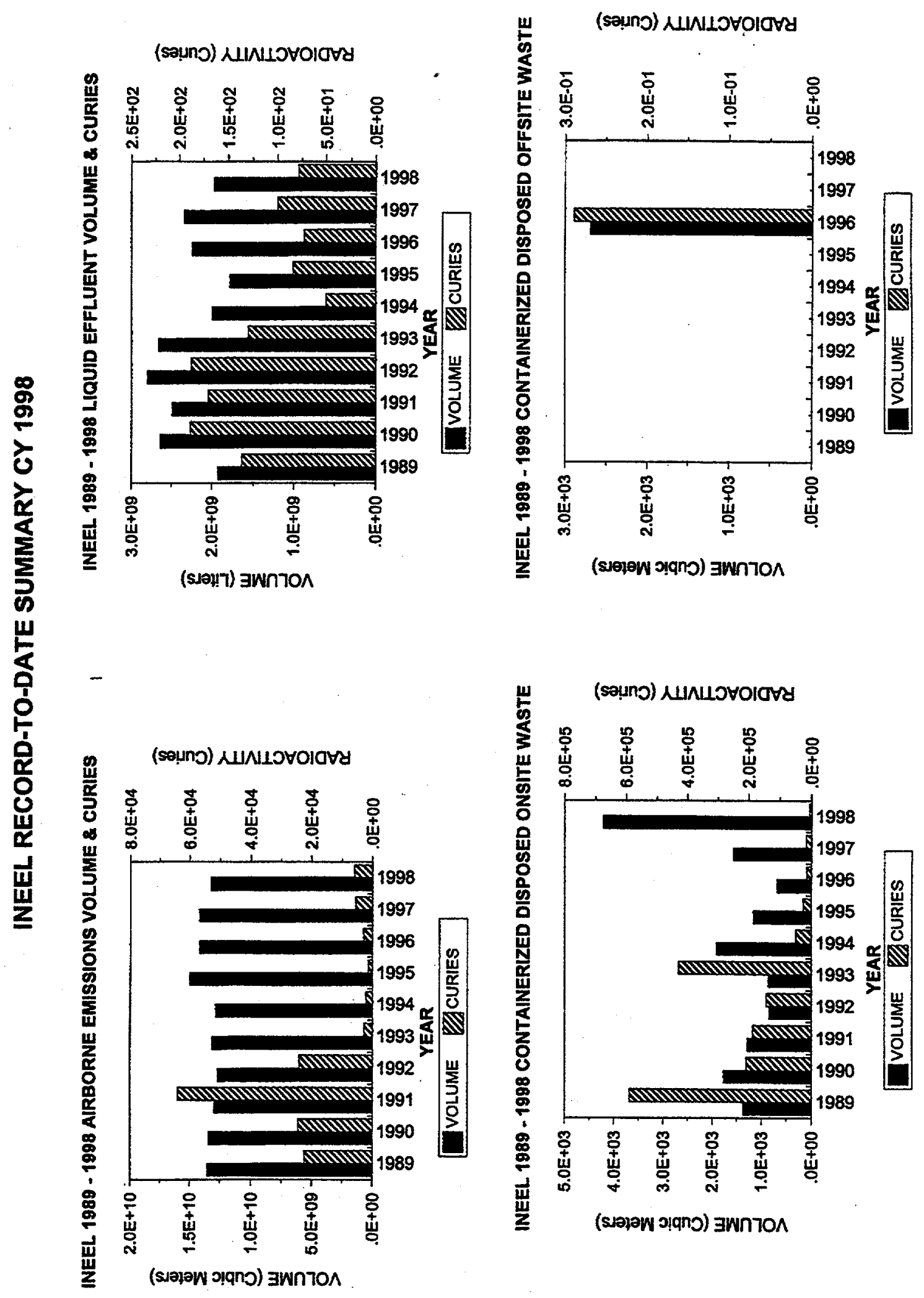


\section{INEEL RECORD-TO-DATE SUMMARY CY 1998}

1989 - 1998 CONTAINERIZED WASTE DISPOSED AT WIPP

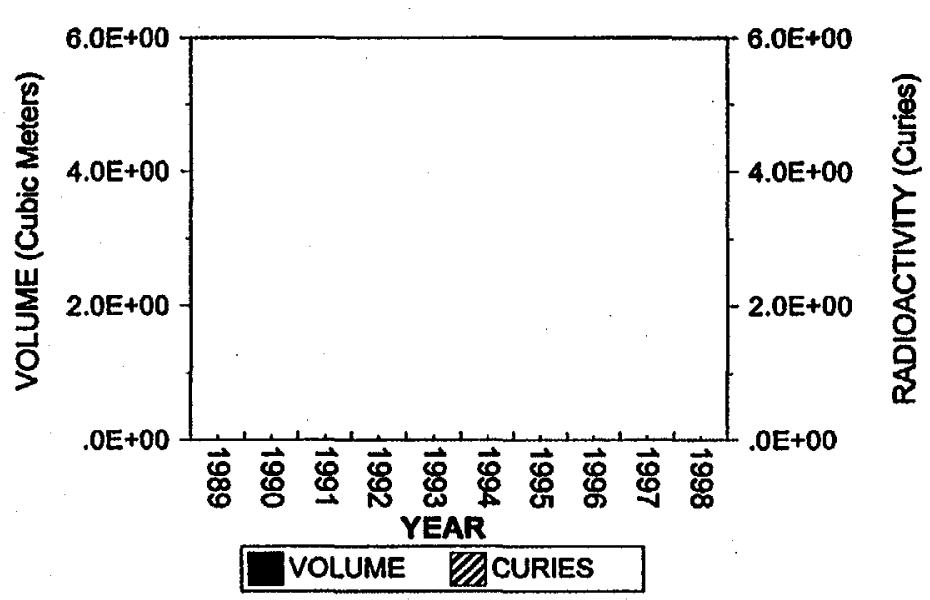

1989 - 1998 CONTAINERIZED WASTE STORED AT TSA / ILT

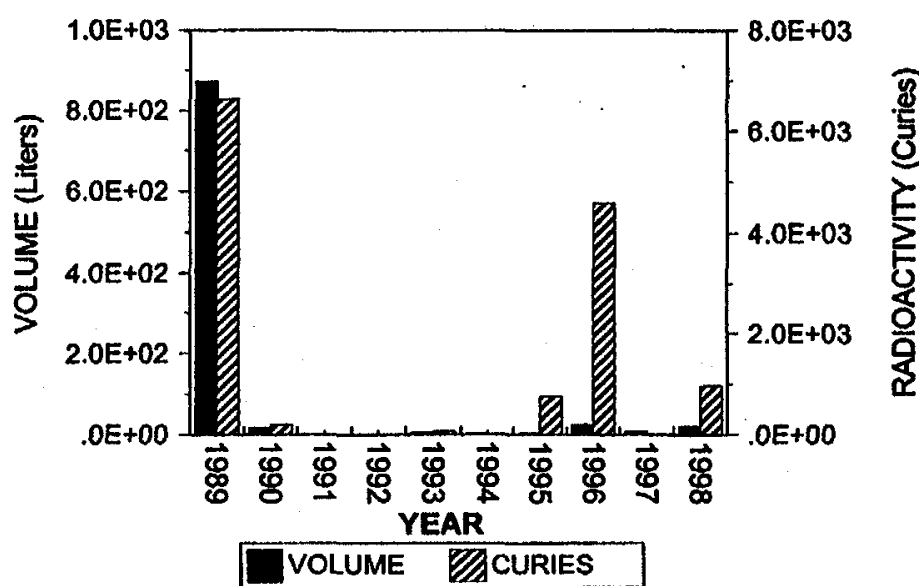

1989 - 1998 CONTAINERIZED STORED WASTE

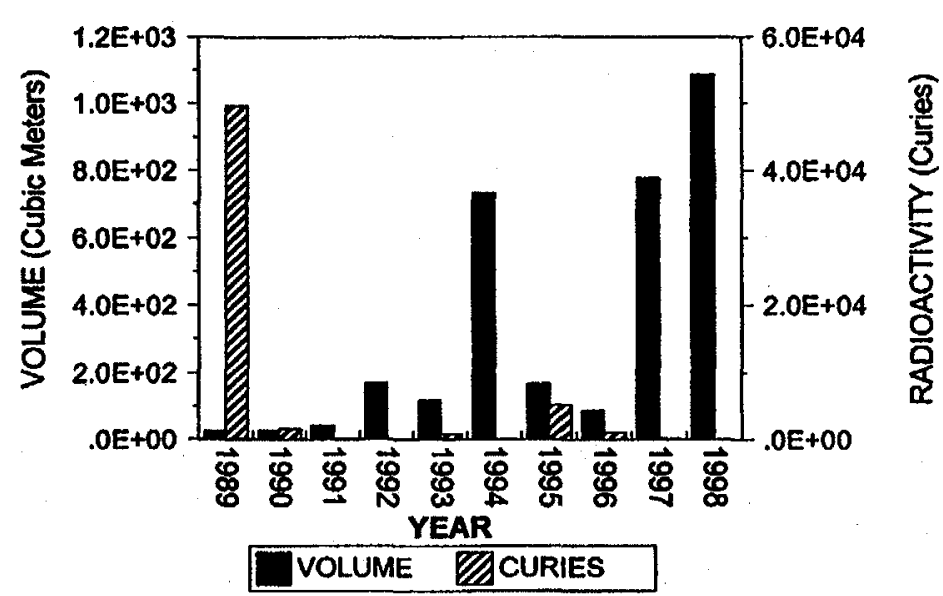

1989 - 1998 CONTAINERIZED RE-EXAMINED BY TRIPS

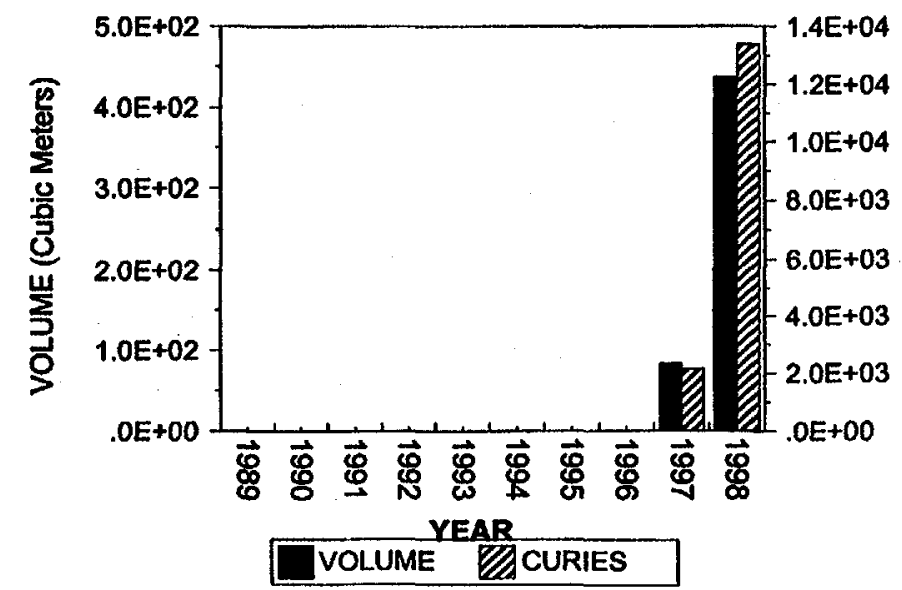


INEEL RECORD-TO-DATE SUMMARY CY 1998

1989 - 1998 INCINERABLE WASTE SENT TO PRIVATE INDUSTRY

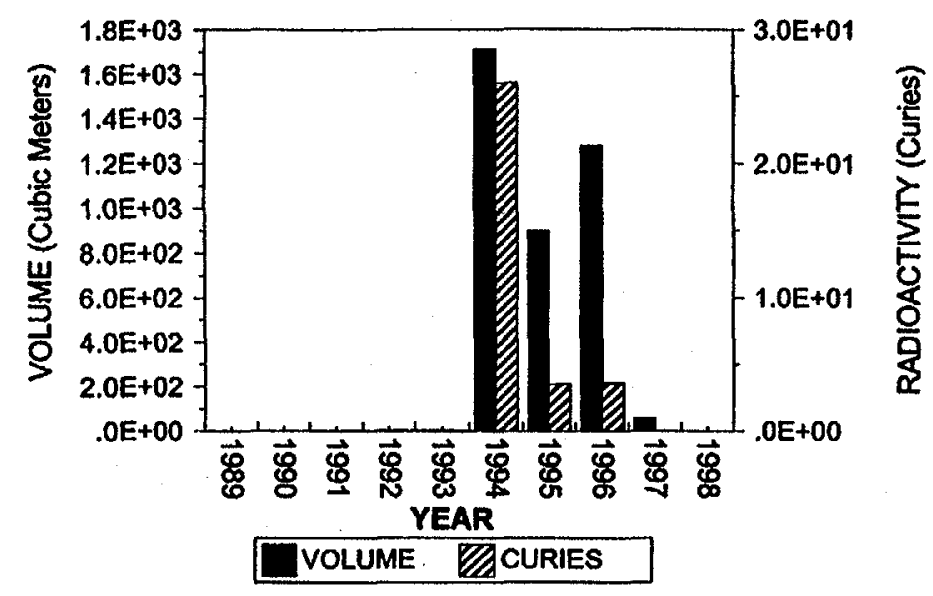

1989 - 1998 CONTAINERIZED WASTE TO WERF FOR VOLUME REDUCTION

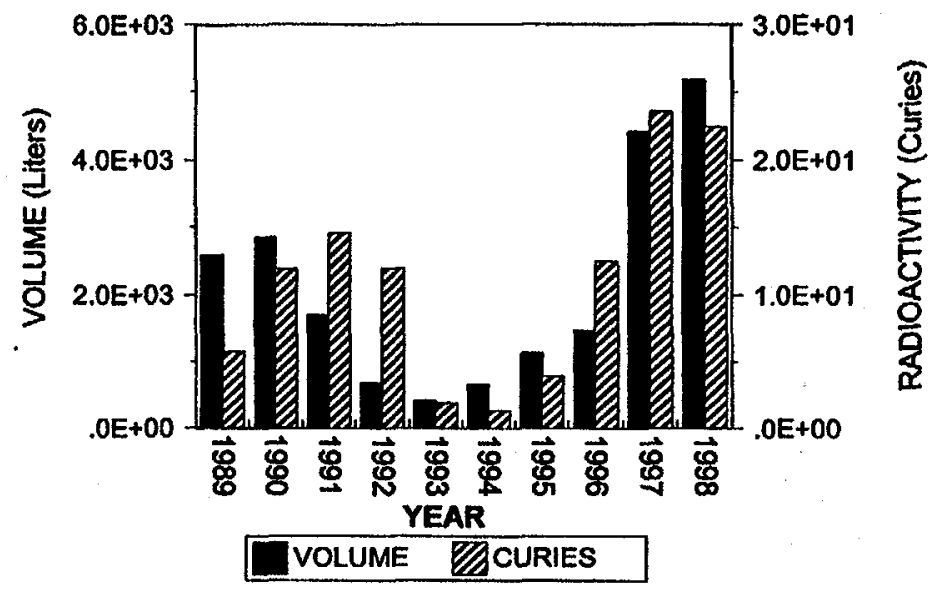

WERF began reducing waste in 1984

1989 - 1998 WERF INVENTORY

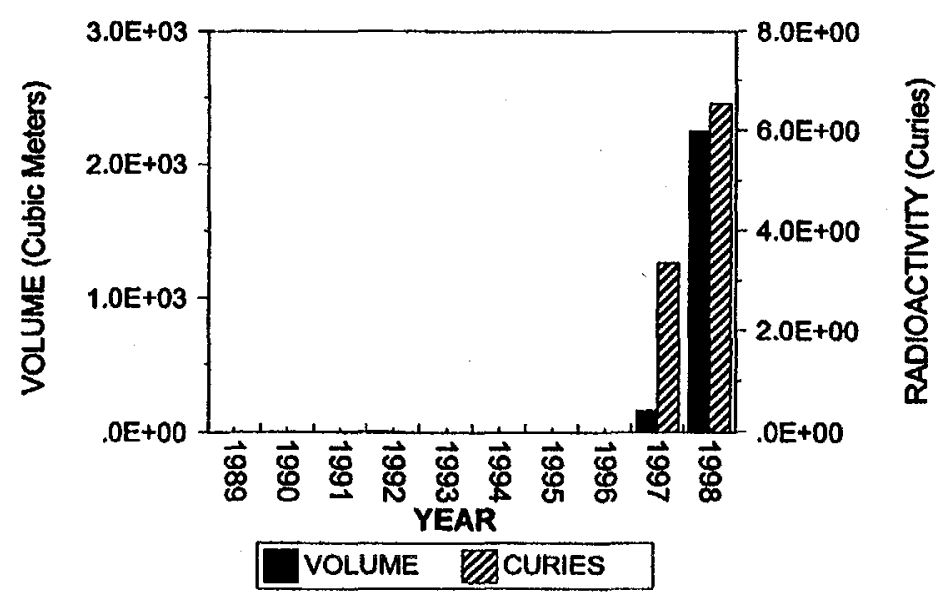




\section{INEEL Record-to-Date Discharges and Solid Waste}

INEEL Radioactive Airborne Waste Discharges, Record-to-Date .................................................. REEL-9

INEEL Radioactive Airborne Waste Discharges Record-to-Date Bar Graphs..................................INEEL-11

INEEL Radioactive Liquid Waste Discharges, Record-To-Date ....................................................INEEL-14

INEEL Radioactive Liquid Waste Discharge Record-To-Date Bar Graphs...................................INEEL-15

INEEL Solid Record-to-Date Summary, Disposed Waste ........................................................INEEL-17

INEEL Solid Record-to-Date Summary Disposed Waste Bar Graphs .............................................INEEL-20

INEEL Solid Record-to-Date Summary, On-Site Stored Waste ....................................................INEEL-25

INEEL Solid Record-to-Date Summary On-Site Stored Waste Bar Graphs ......................................INEEL-26

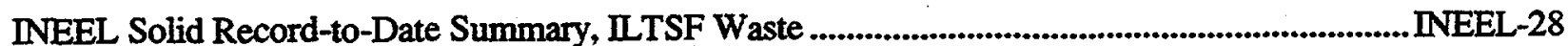

INEEL Solid Record-to-Date Summary ILTSF Waste Bar Graphs ................................................INEEL-29

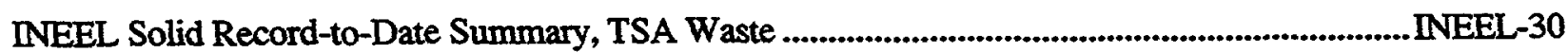

INEEL Solid Record-to-Date Summary TSA Waste Bar Graphs ................................................INEEL-32

INEEL Solid Record-to-Date Summary, WERF Waste..........................................................INEEL-34

INEEL Solid Record-to-Date Summary WERF Waste Bar Graphs.................................................INEEL-36 


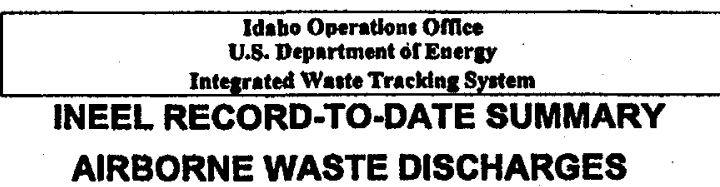

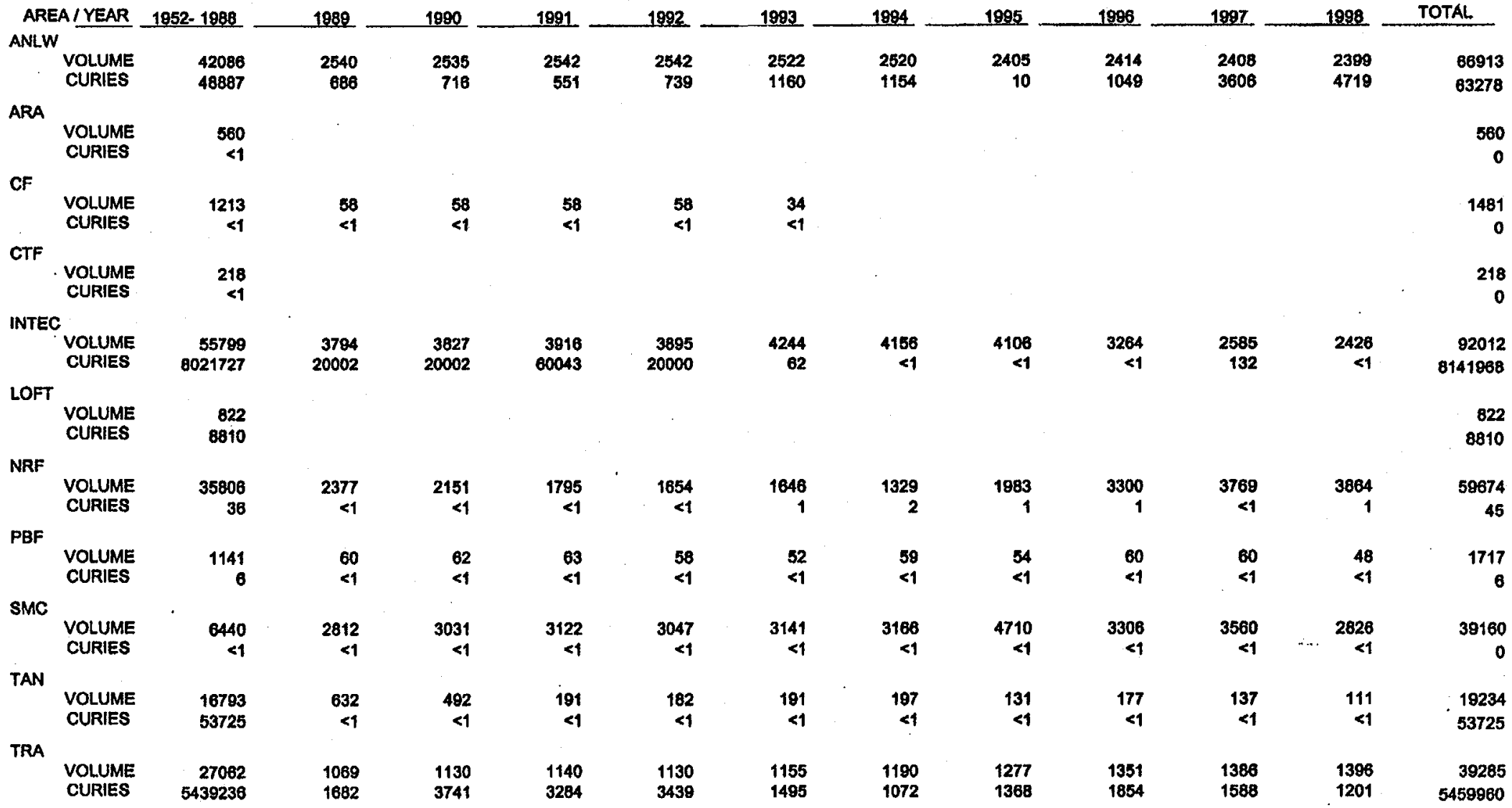




\begin{tabular}{|c|c|c|c|c|c|c|c|c|c|c|c|c|}
\hline \multirow{2}{*}{ AREA /YEAR } & & \multirow[b]{2}{*}{1989} & \multirow[b]{2}{*}{1990.} & \multicolumn{5}{|c|}{ 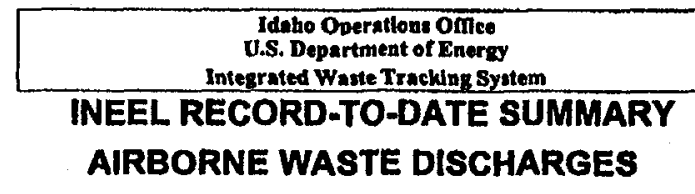 } & \multirow{2}{*}{1808} & \multirow[b]{2}{*}{1897.} & \multirow[b]{2}{*}{1898} & \multirow[b]{2}{*}{ TOTAL } \\
\hline & $1952-1988$ & & & 1991 & 1992 & 1993 & 1994. & 1995. & & & & \\
\hline $\begin{array}{l}\text { WERF } \\
\text { VOLUME } \\
\text { CURIES }\end{array}$ & $\begin{array}{r}249 \\
<1\end{array}$ & $\begin{array}{r}150 \\
<1\end{array}$ & $\begin{array}{r}164 \\
<1\end{array}$ & $\begin{array}{r}137 \\
<1\end{array}$ & .135 & $\begin{array}{r}155 \\
<1\end{array}$ & $\begin{array}{r}239 \\
4\end{array}$ & $\begin{array}{r}301 \\
<1\end{array}$ & $\begin{array}{r}302 \\
<1\end{array}$ & $\begin{array}{l}283 \\
<1\end{array}$ & $\begin{array}{r}173 \\
<1\end{array}$ & $\begin{array}{r}2294 \\
0\end{array}$ \\
\hline $\begin{array}{l}\text { WMF VOLUME } \\
\text { VOURIES }\end{array}$ & $\begin{array}{l}13 \\
<1\end{array}$ & $\begin{array}{l}15 \\
<1\end{array}$ & & & $\begin{array}{l}14 \\
<1\end{array}$ & $\begin{array}{l}10 \\
<1\end{array}$ & & & & & & $\begin{array}{r}52 \\
0\end{array}$ \\
\hline $\begin{array}{l}\text { TOTAL VOLUME } \\
\text { CURIES }\end{array}$ & $\begin{array}{r}188202 \\
13572428\end{array}$ & $\begin{array}{l}13514 \\
22370\end{array}$ & $\begin{array}{l}13450 \\
24459\end{array}$ & $\begin{array}{l}12863 \\
63678\end{array}$ & $\begin{array}{l}12715 \\
24179\end{array}$ & $\begin{array}{r}13150 \\
2719\end{array}$ & $\begin{array}{r}12856 \\
2228\end{array}$ & $\begin{array}{r}14968 \\
1380\end{array}$ & $\begin{array}{r}14174 \\
2804\end{array}$ & $\begin{array}{r}14187 \\
5327\end{array}$ & $\begin{array}{r}13242 \\
5921\end{array}$ & $\begin{array}{r}323421 \\
13,727,794\end{array}$ \\
\hline
\end{tabular}




\section{INEEL RADIOACTIVE AIRBORNE WASTE DISCHARGES RECORD-TO-DATE}

ANLW 1989 - 1998 AIRBORNE EMISSION VOLUME \& CURIES

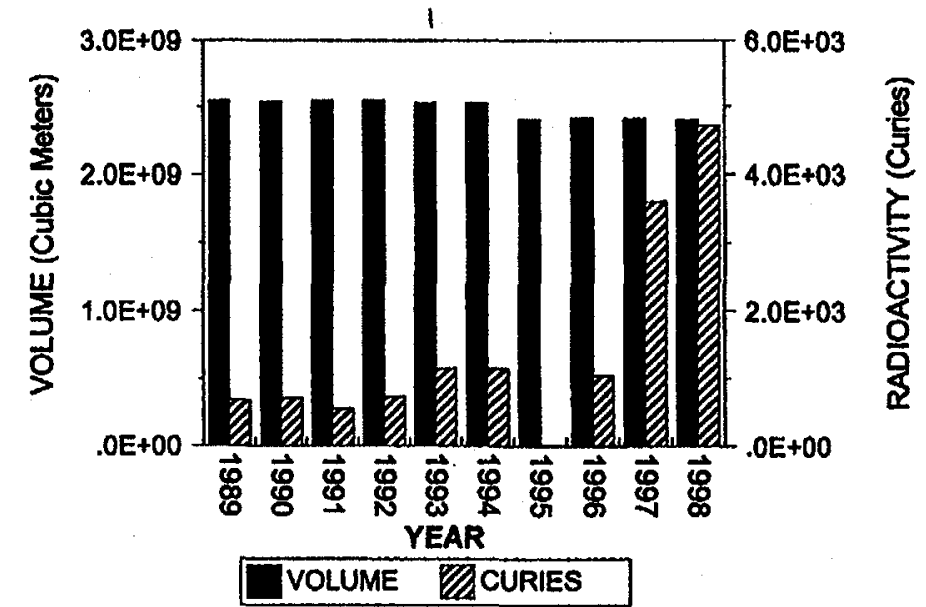

CF 1989 - 1998 AIRBORNE EMISSION VOLUME \& CURIES

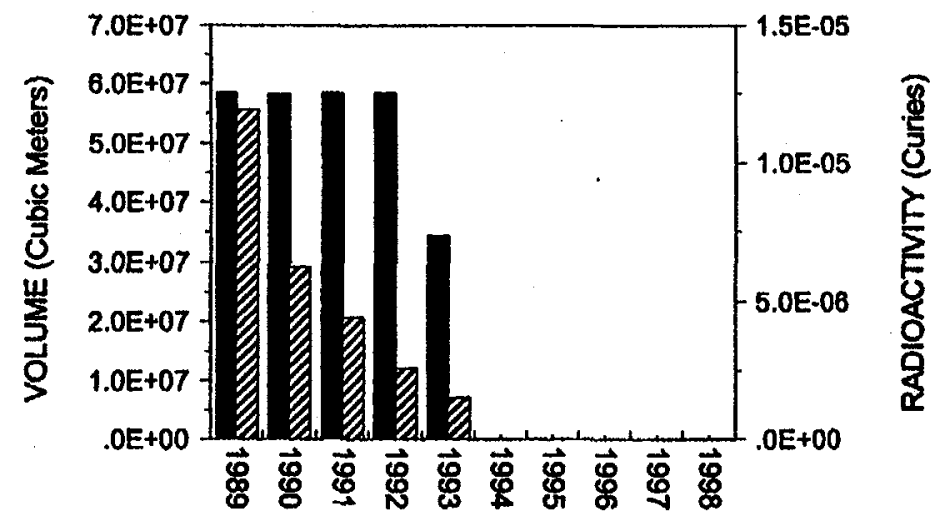

Graph: G22DA01
INTEC 1989 - 1998 AIRBORNE EMISSION VOLUME \& CURIES

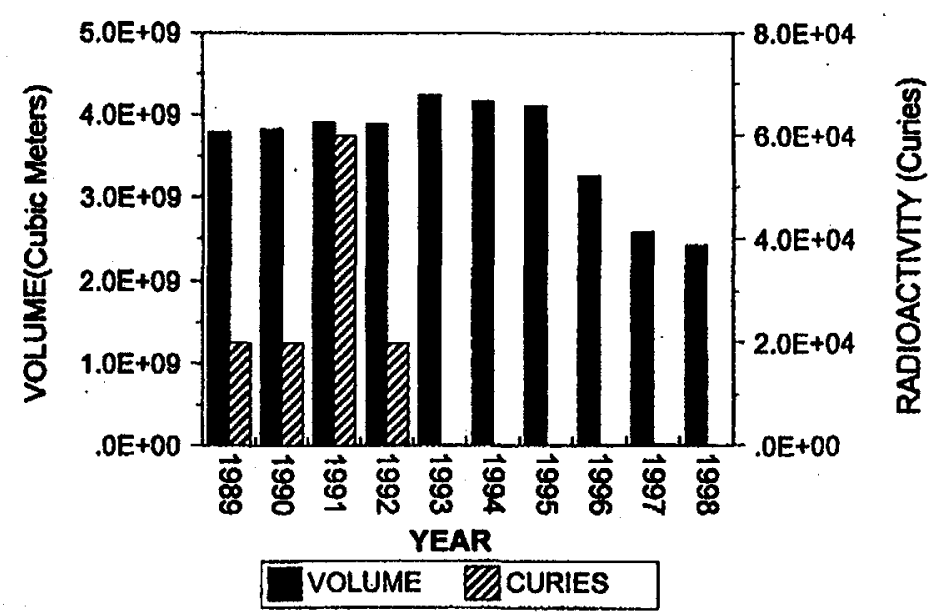




\section{INEEL RADIOACTIVE AIRBORNE WASTE DISCHARGES RECORD-TO-DATE}

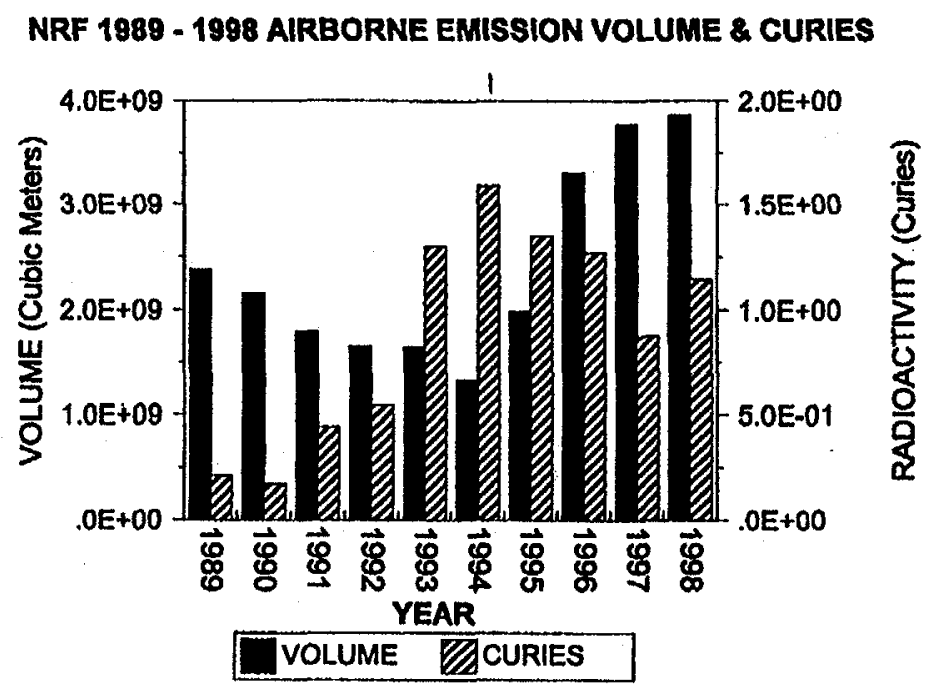

PBF 1989 - 1998 AIRBORNE EMISSION VOLUME \& CURIES

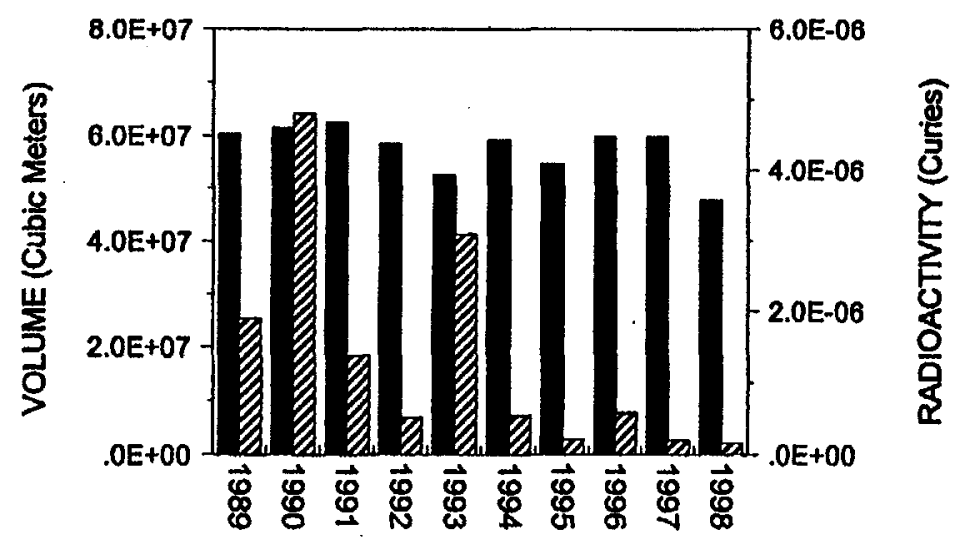

Graph: G22DA02
YEAR
SMC 1989 - 1998 AIRBORNE EMISSION VOLUME \& CURIES

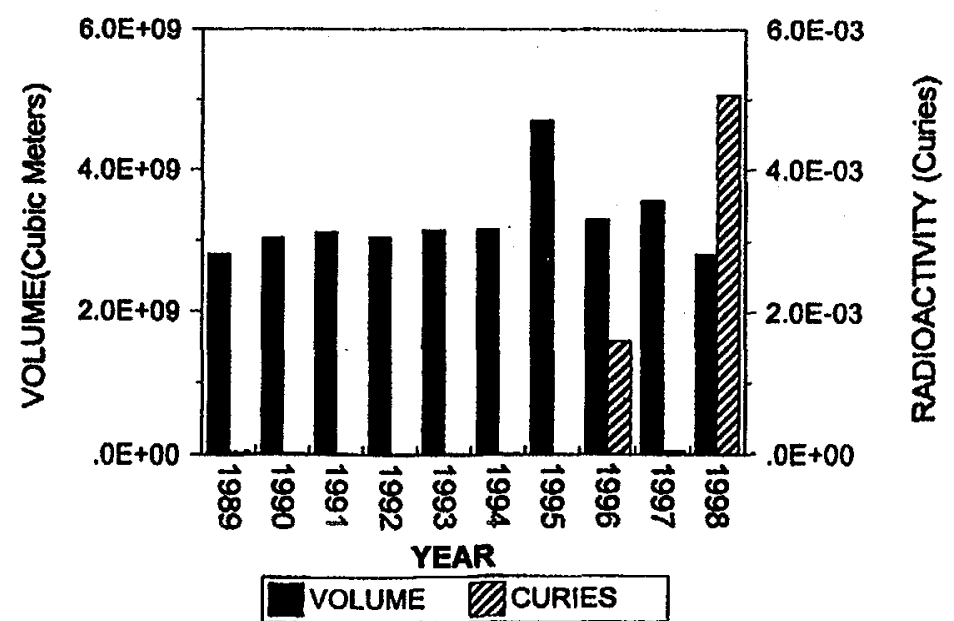




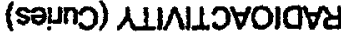
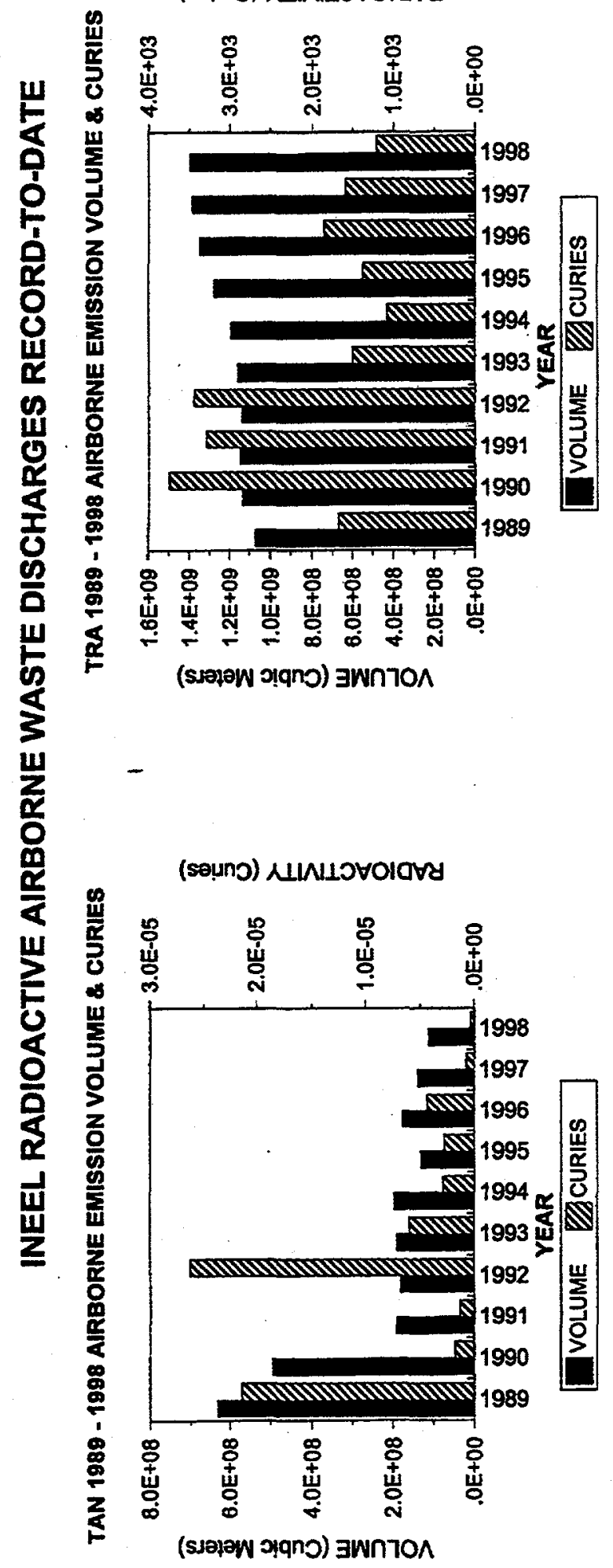

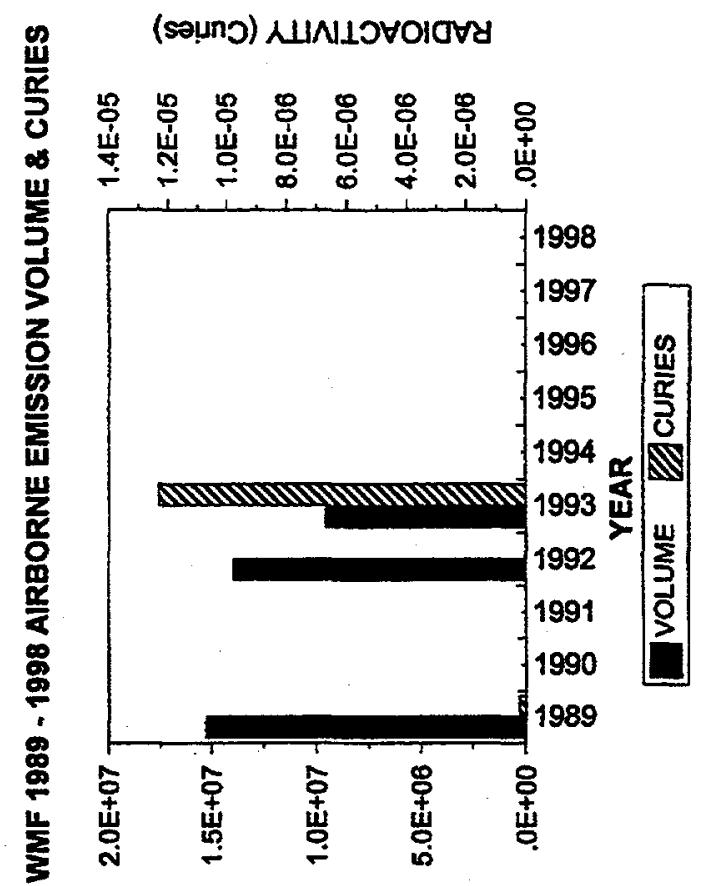

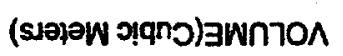

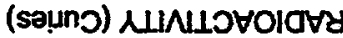

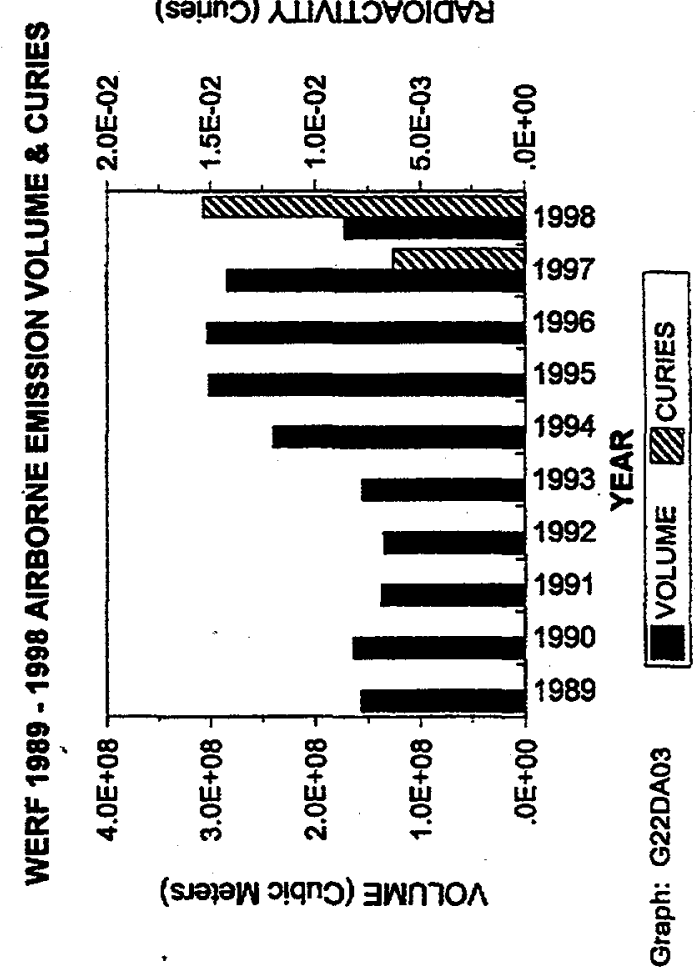




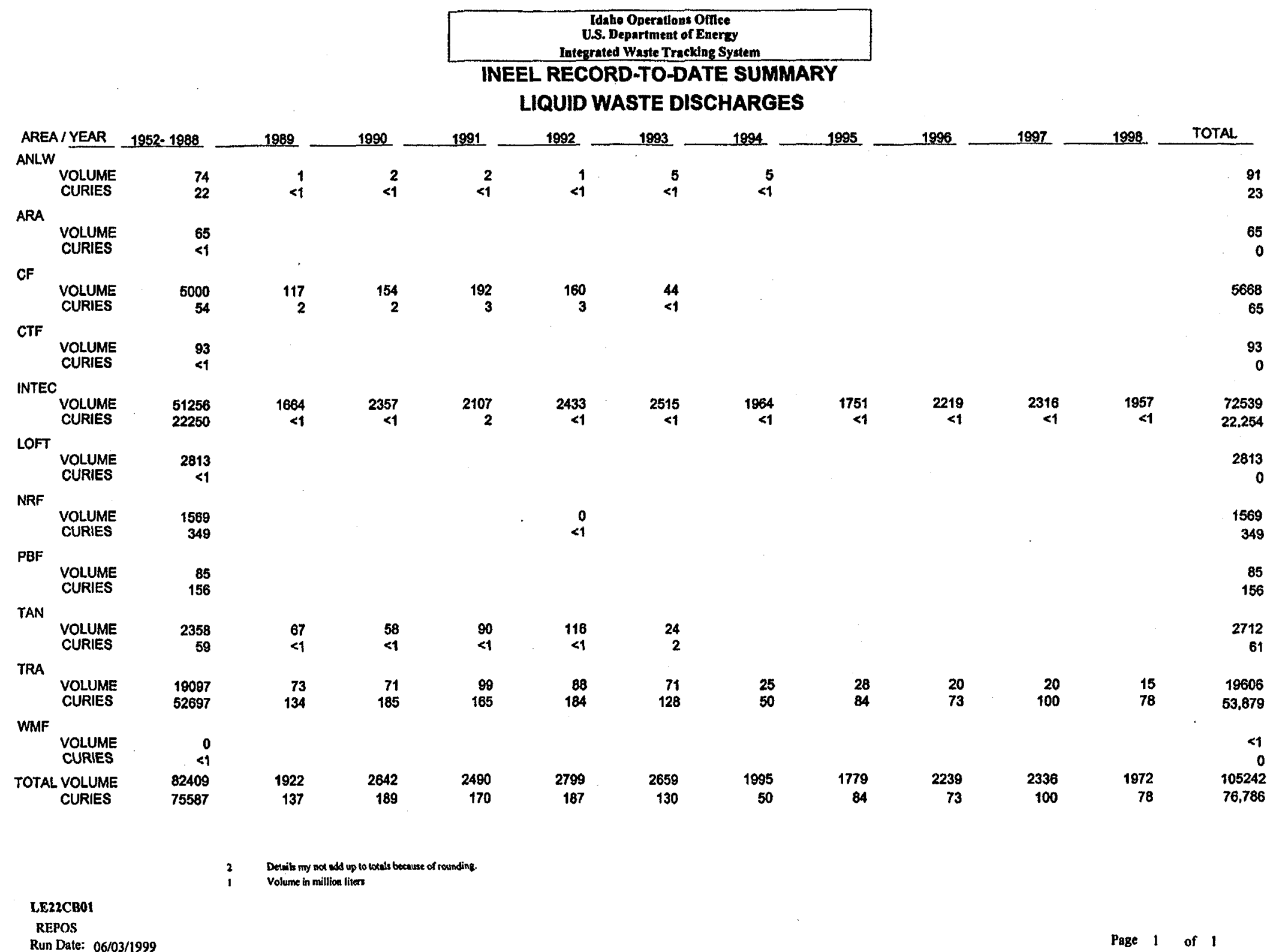



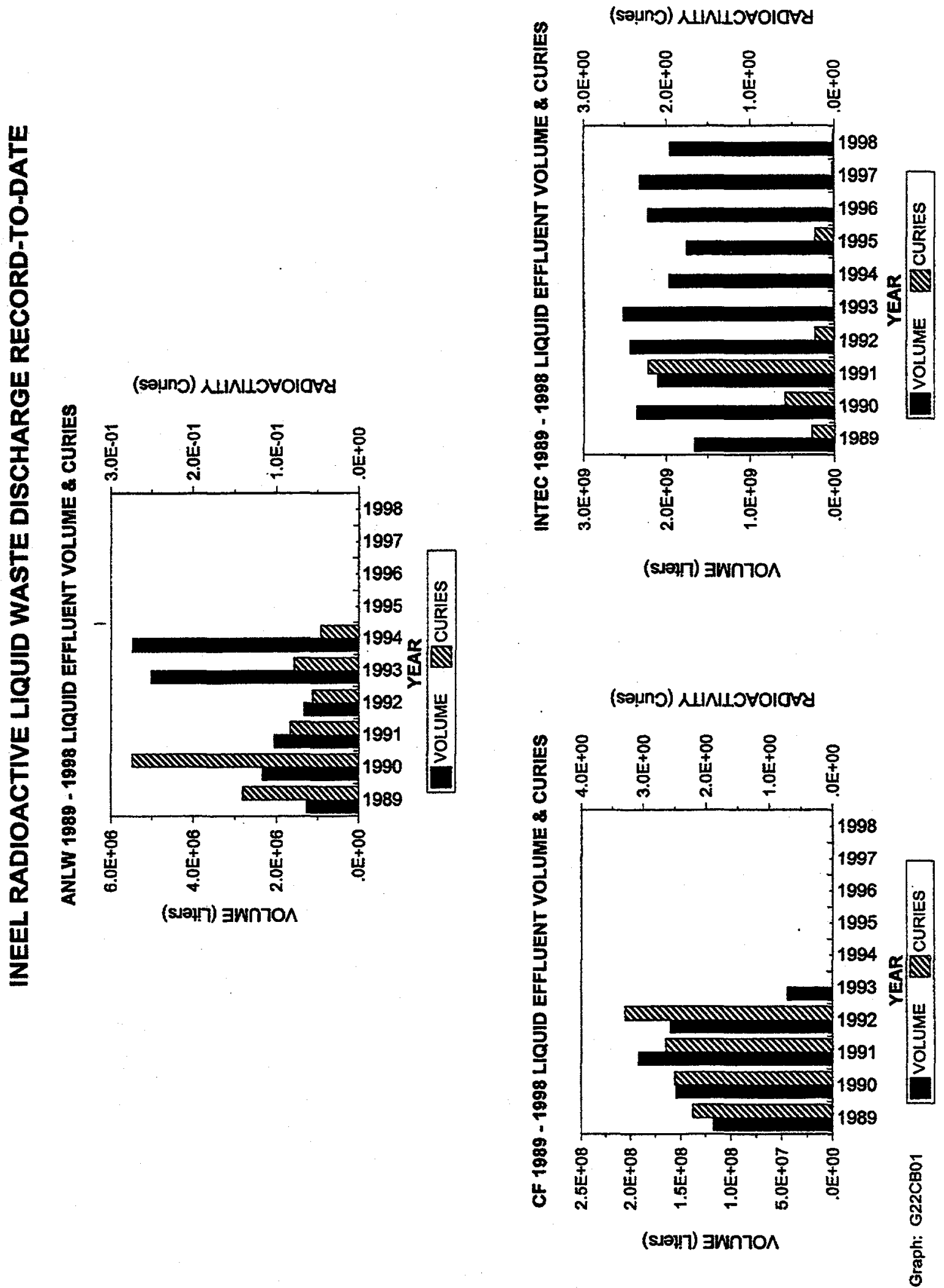


\section{INEEL RADIOACTIVE LIQUID WASTE DISCHARGE RECORD-TO-DATE}

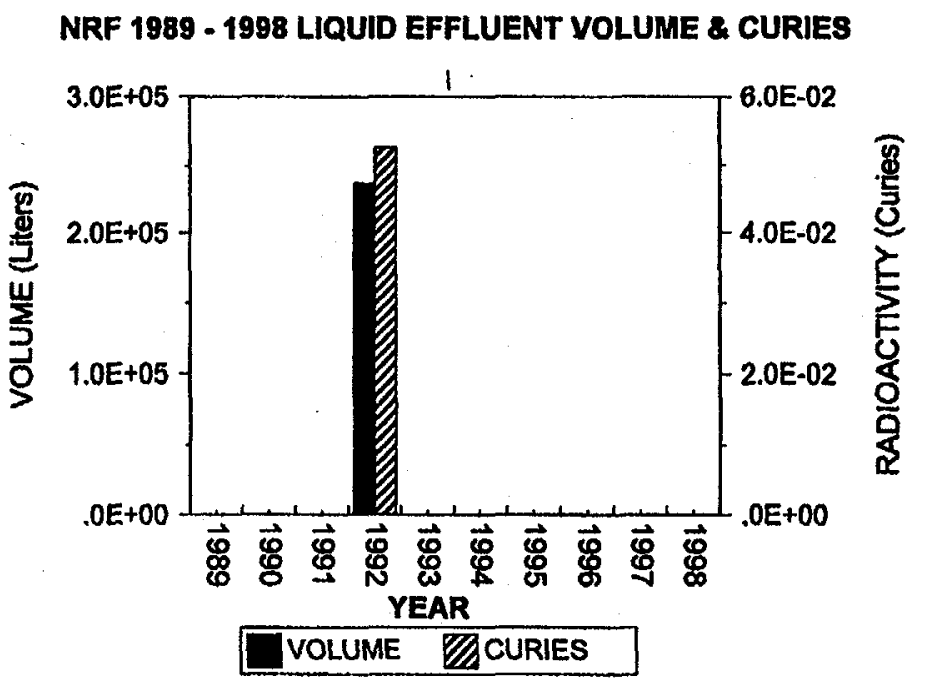

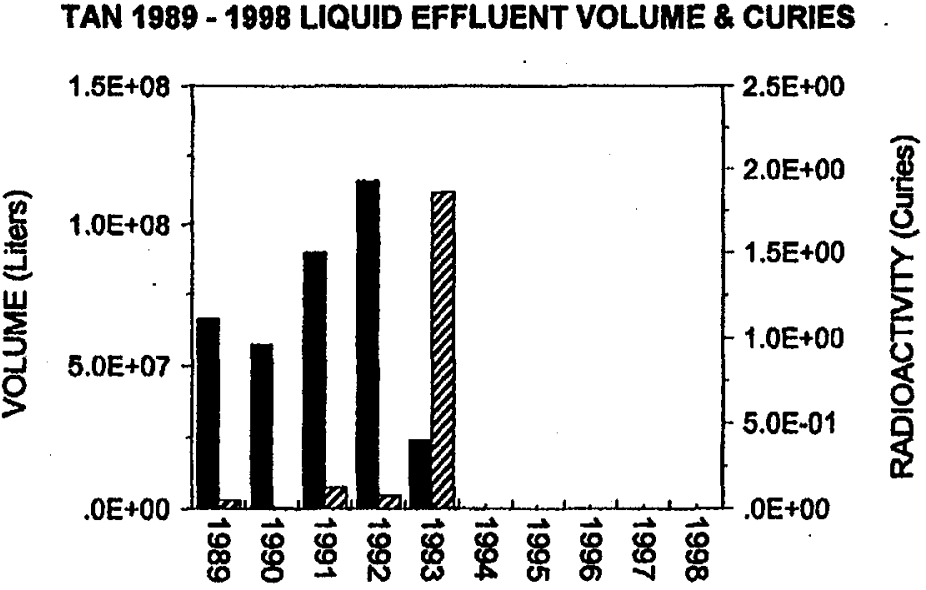

Graph: G22CB02

YEAR
TRA 1989 - 1998 LIQUID EFFLUENT VOLUME \& CURIES

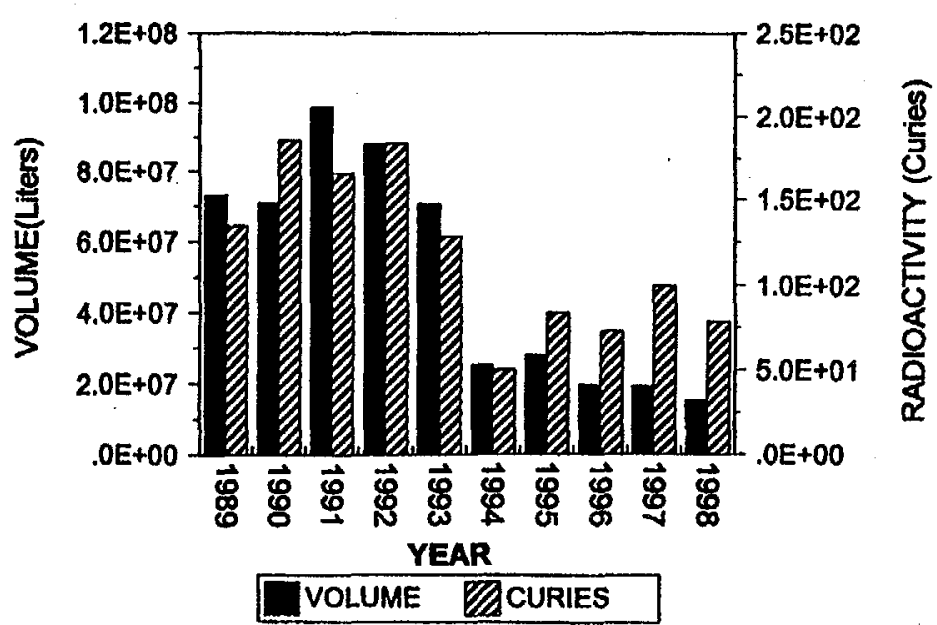




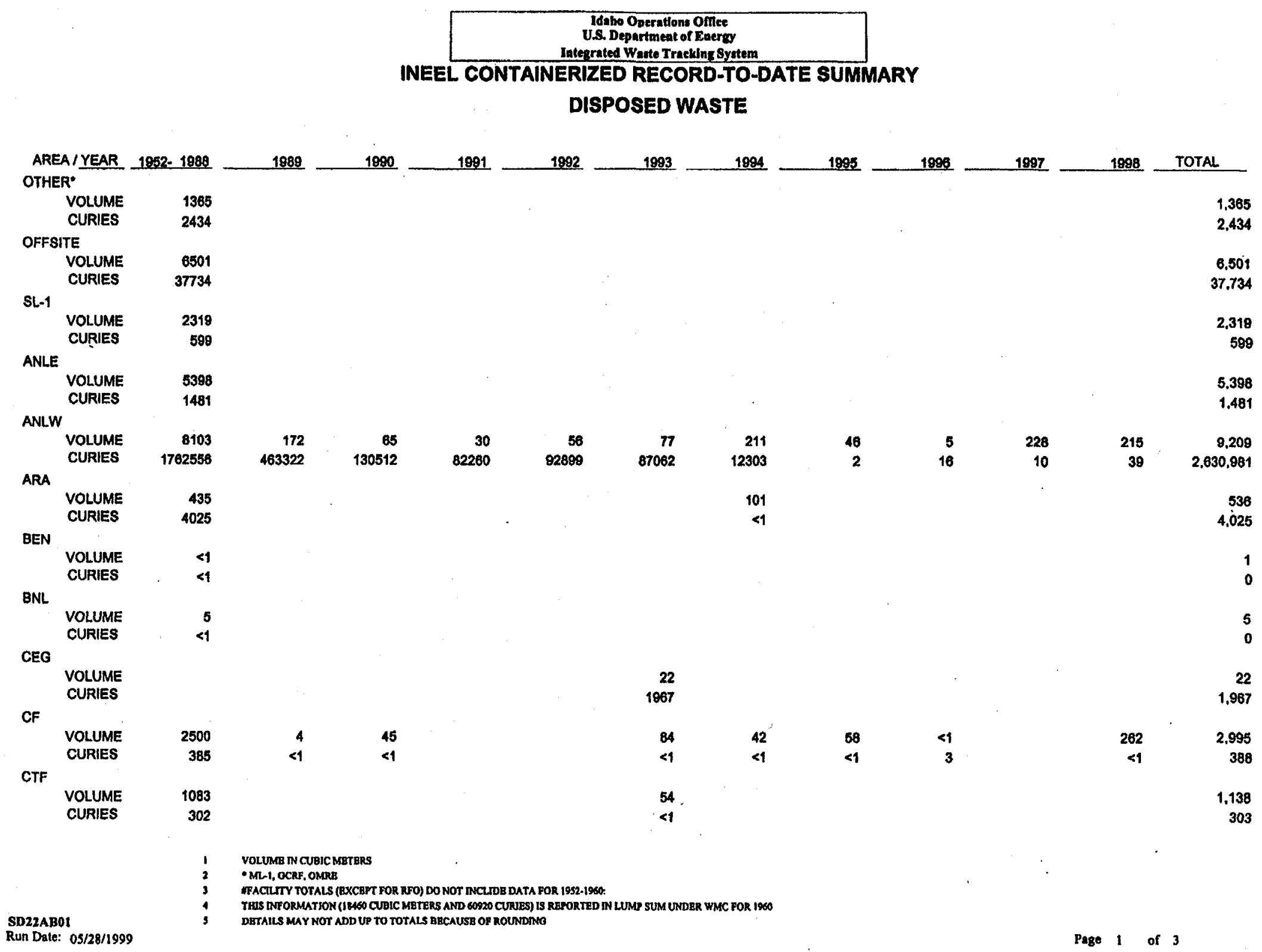




\section{Idaho Operatlons Omee
U.S. Department of Energ \\ Utemated wate Track \\ INEEL CONTAINERIZED RECORD-TO-DATE SUMMARY \\ DISPOSED WASTE}

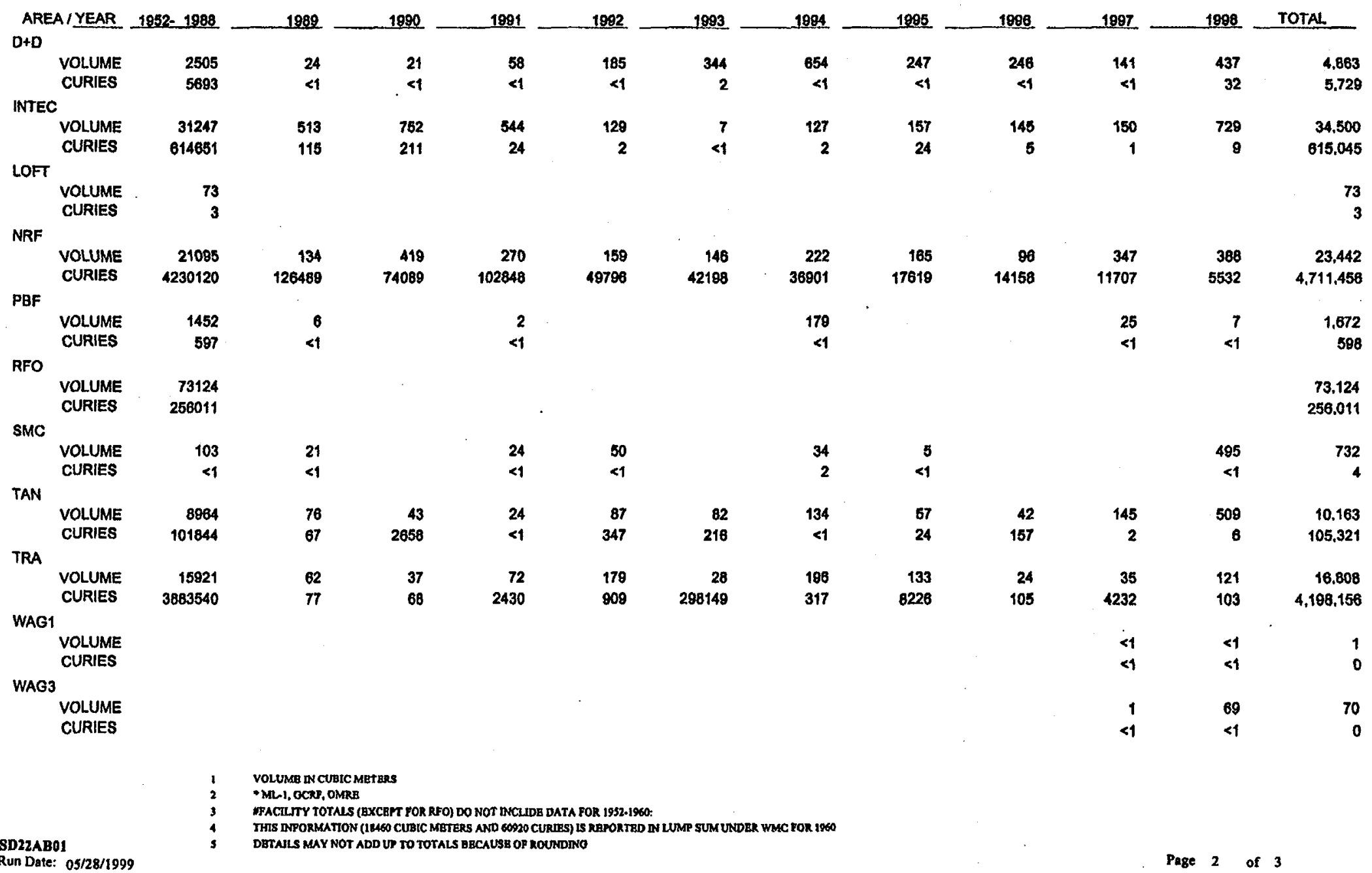




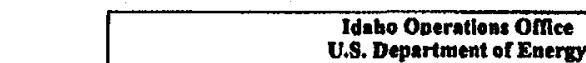 \\ U.S. Department of Energy \\ INEEL CONTAINERIZED RECORD-TO-DATE SUMMARY \\ DISPOSED WASTE}

\begin{tabular}{|c|c|c|c|c|c|c|c|c|c|c|c|c|}
\hline \multirow{2}{*}{\multicolumn{13}{|c|}{$\begin{array}{l}\text { AREA / YEAR 1952-1988 } \\
\text { WAG7 }\end{array}$}} \\
\hline & & & & & & & & & & & & \\
\hline VOLUME & & & & & & & & & & & 25 & 25 \\
\hline CURIES & & & & & & & & & & & $<1$ & 0 \\
\hline \multicolumn{13}{|l|}{ WERF } \\
\hline VOLUME & 918 & 328 & 359 & 248 & & 7 & 5 & 289 & 111 & 489 & 968 & 3.722 \\
\hline CURIES & $<1$ & $<1$ & 6 & 3 & & $<1$ & $<1$ & 10 & 1 & 10 & 17 & 57 \\
\hline \multicolumn{13}{|l|}{ WMF } \\
\hline VOLUME & 19980 & 23 & 22 & & & & & 2 & 6 & & & 20.033 \\
\hline CURIES & 60927 & $<1$ & $<1$ & & & & & $<1$ & $<1$ & & & 60,927 \\
\hline $\begin{array}{c}\text { TOTAL VOLUME } \\
\text { CURIES }\end{array}$ & $\begin{array}{r}203094 \\
10962904\end{array}$ & $\begin{array}{r}1384 \\
590070\end{array}$ & $\begin{array}{r}1762 \\
207544\end{array}$ & $\begin{array}{r}1272 \\
107565\end{array}$ & $\begin{array}{r}844 \\
143953\end{array}$ & $\begin{array}{r}852 \\
429594\end{array}$ & $\begin{array}{r}1906 \\
49526\end{array}$ & $\begin{array}{r}1159 \\
25907\end{array}$ & $\begin{array}{r}677 \\
14445\end{array}$ & $\begin{array}{r}1564 \\
15972\end{array}$ & $\begin{array}{l}4224 \\
5738\end{array}$ & $\begin{array}{r}218,718 \\
12,633,217\end{array}$ \\
\hline
\end{tabular}


(soụno) ㅅN

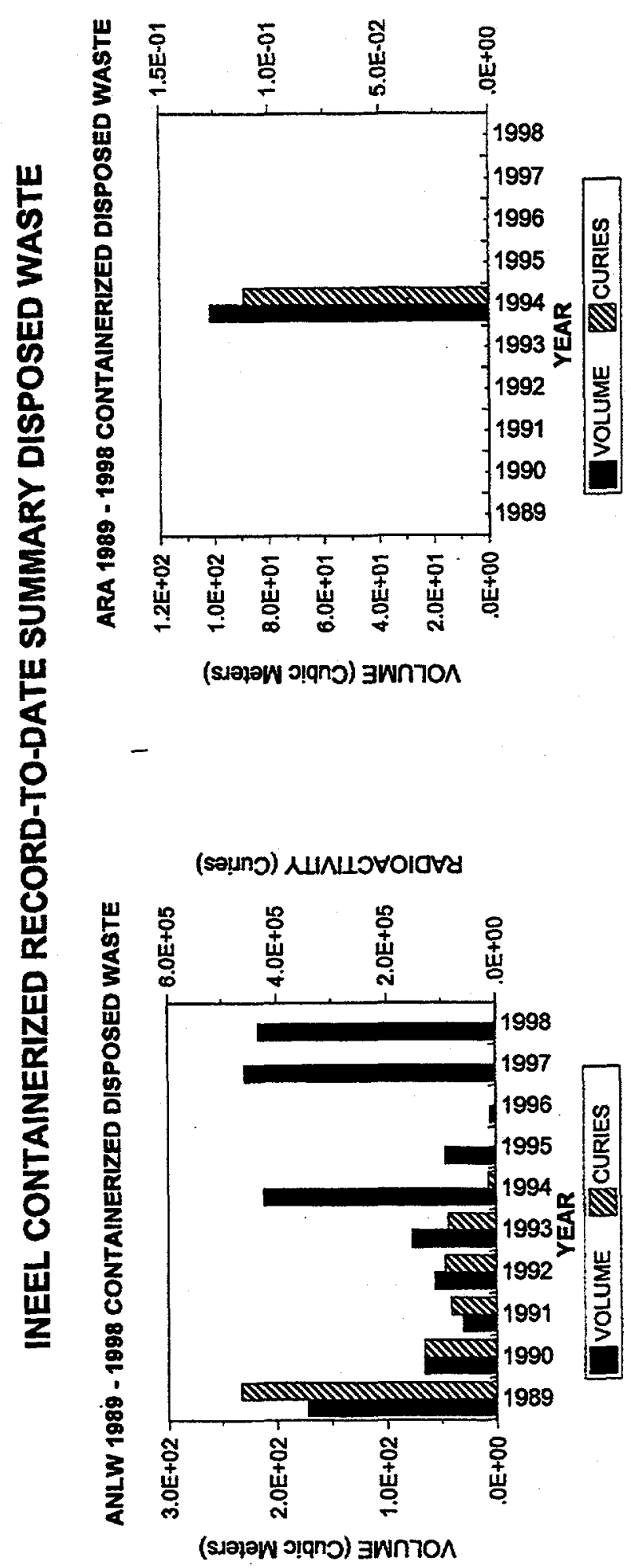

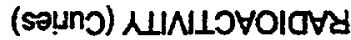

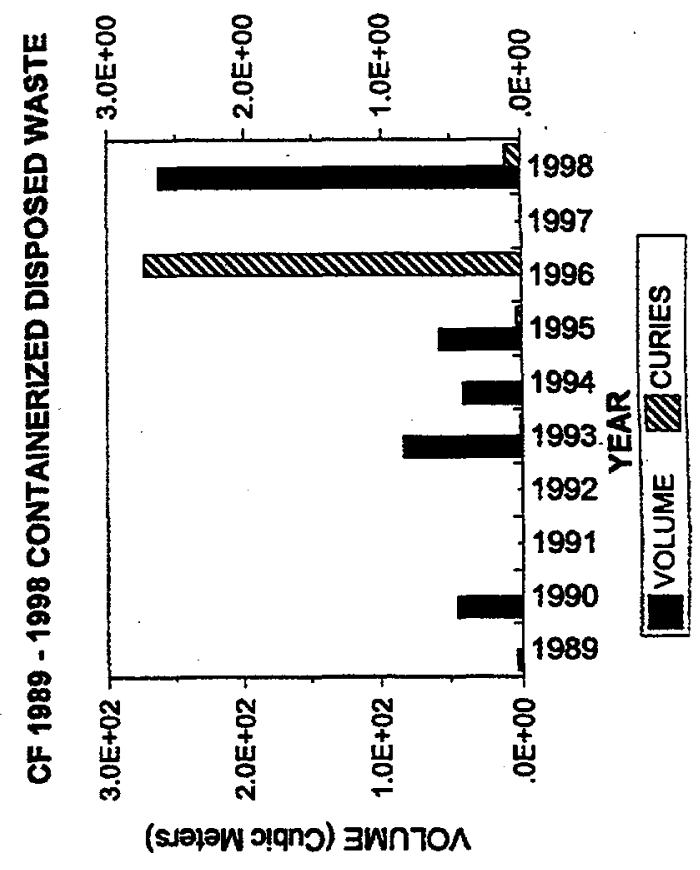

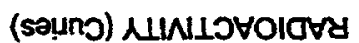

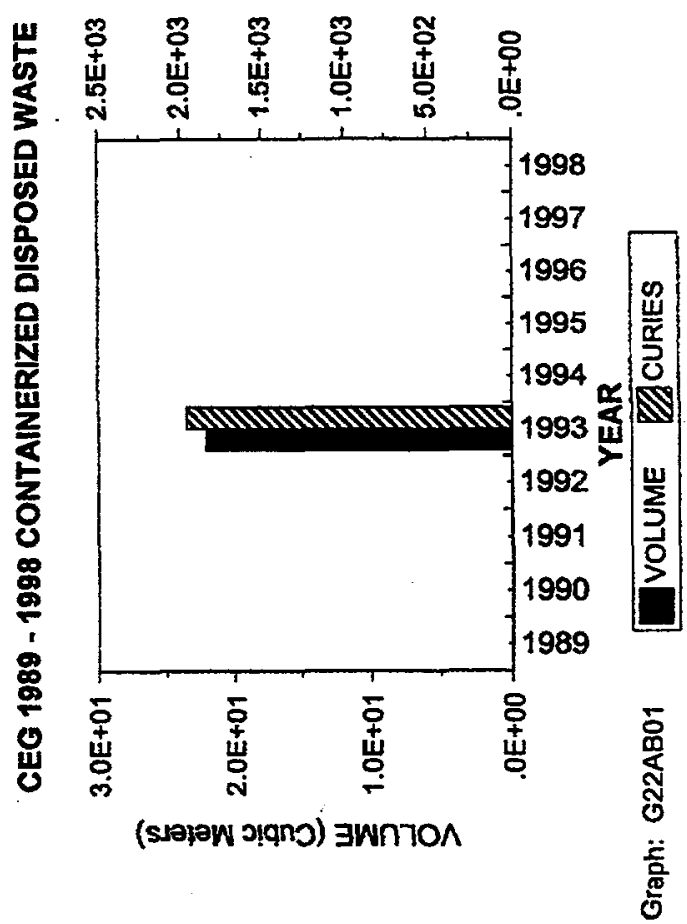


(sạnก) ᄉLINILOVOIOVA

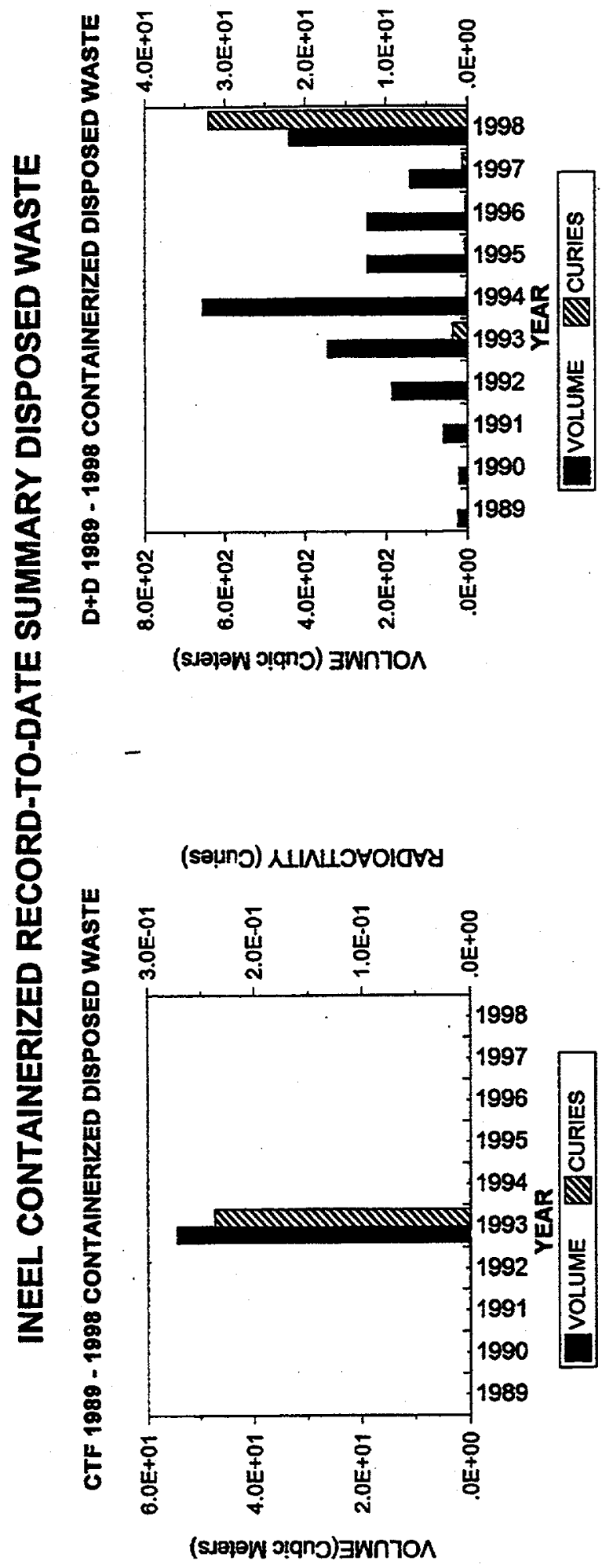

(seunn) ᄉLINLOVOIAVY

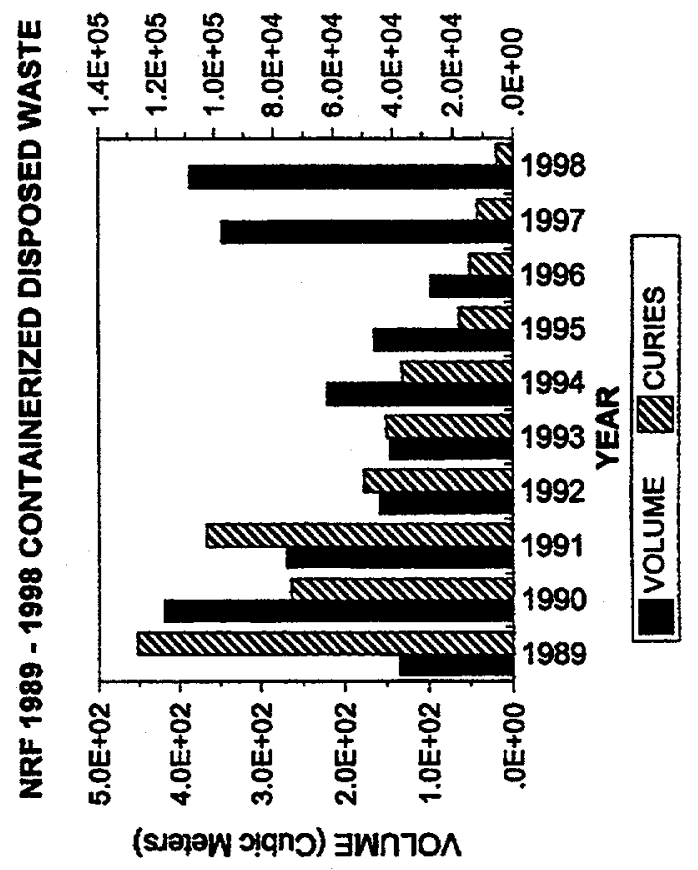

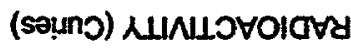

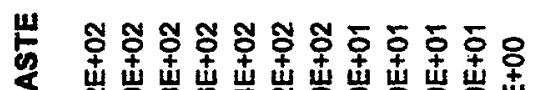

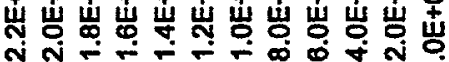

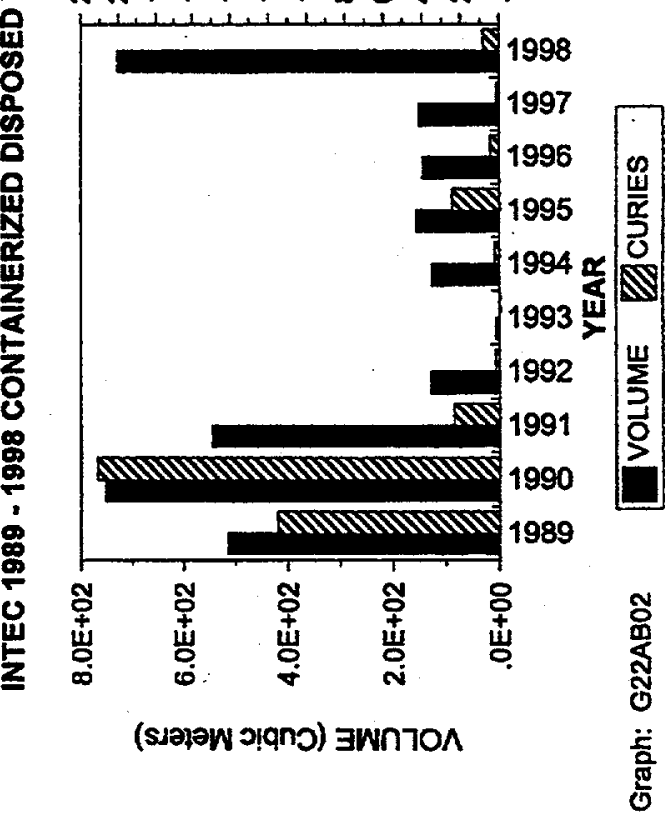



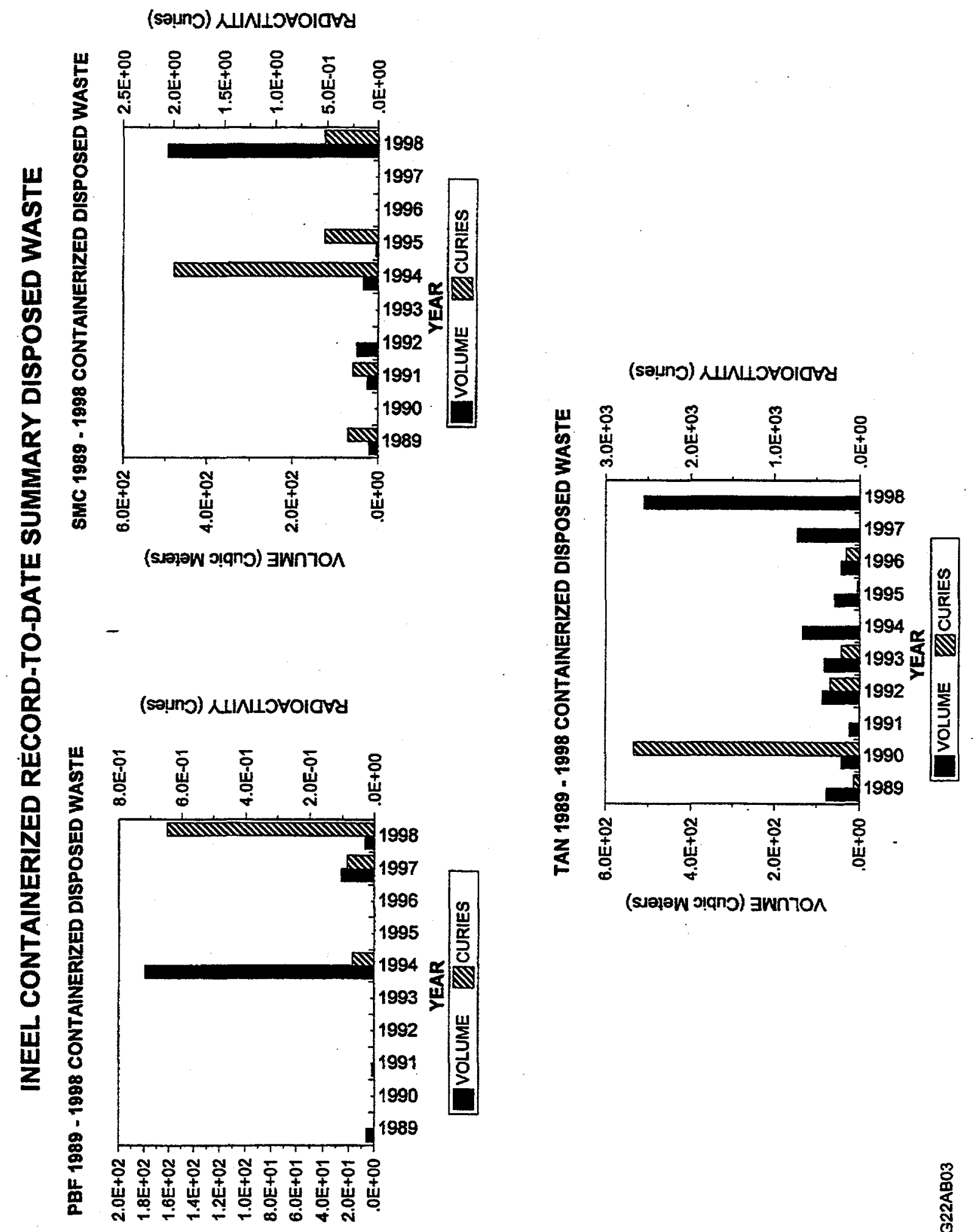

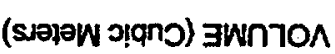


(souno) NUNLOHOIOVY

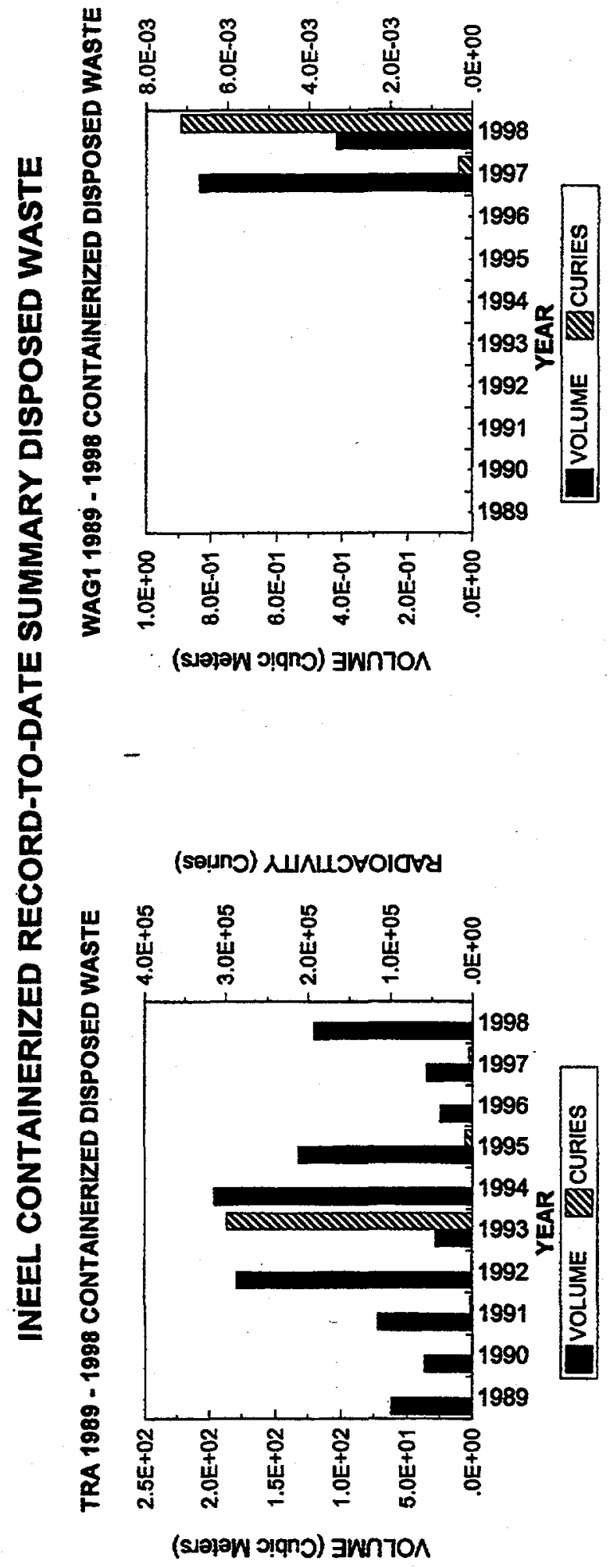

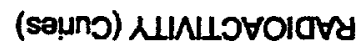
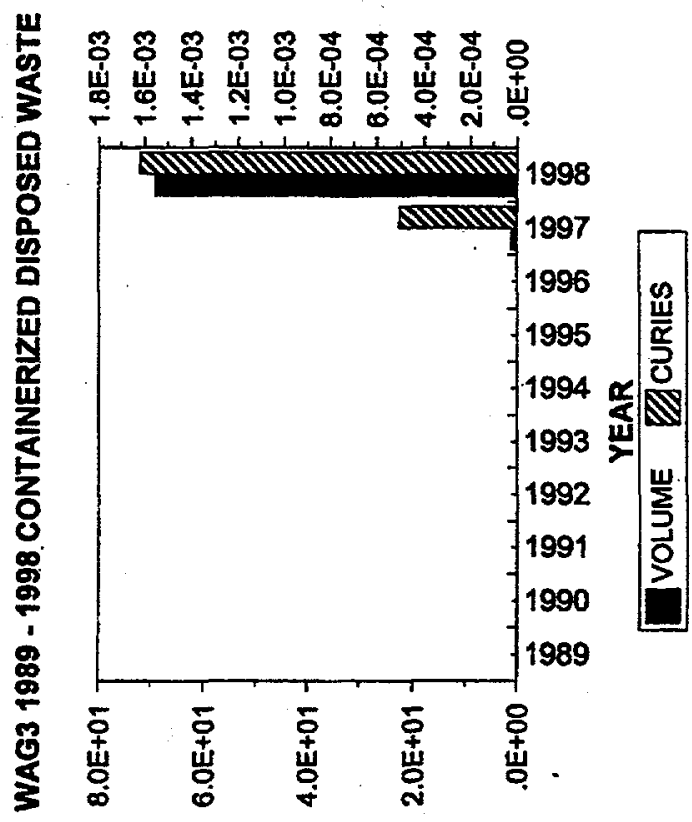

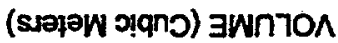




\section{INEEL CONTAINERIZED RECORD-TO-DATE SUMMARY DISPOSED WASTE}

WAG7 1989 - 1998 CONTAINERIZED DISPOSED WASTE

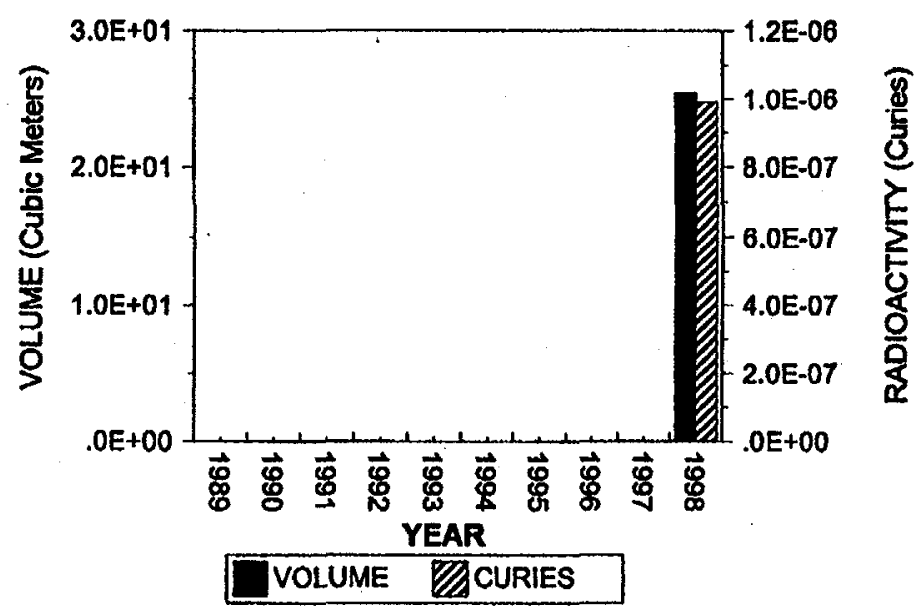

WERF 1989 - 1998 CONTAINERIZED DISPOSED WASTE

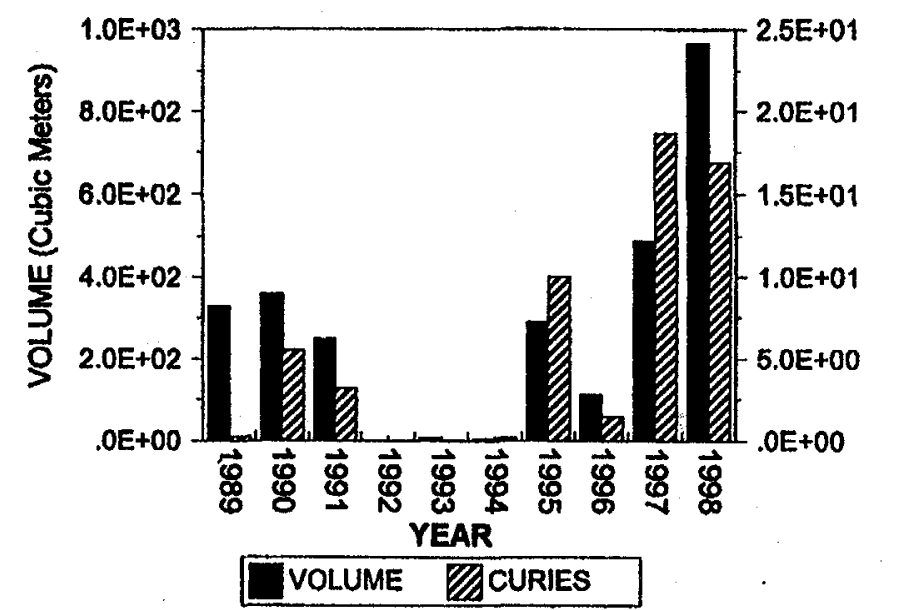

WMF 1989 - 1998 CONTAINERIZED DISPOSED WASTE

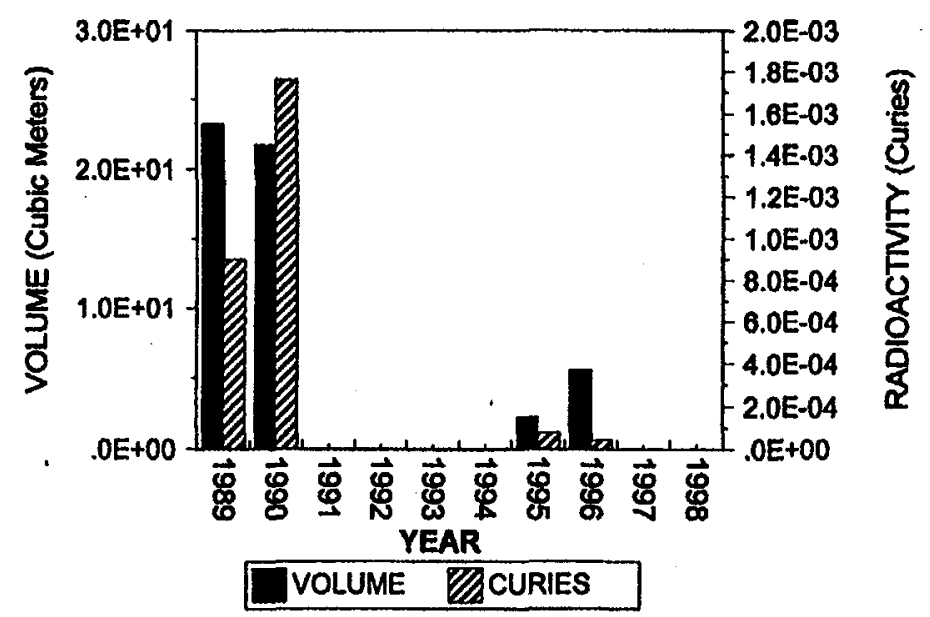




\section{Idaho Operatloas OMce
U.S. Depurtment of Enes \\ Inserrnted Waste Tracking System \\ INEEL CONTAINERIZED RECORD-TO-DATE SUMMARY \\ ONSITE STORED WASTE}

\begin{tabular}{|c|c|c|c|c|c|c|c|c|c|c|c|c|}
\hline AREA / YEAR & $1952-1988$ & 1889 & 1990 & 1991 & 1992 & 1993 & 1994. & 1995 & 1096 & 1997 & 1898 & TOTAL \\
\hline \multicolumn{13}{|l|}{ ANLW } \\
\hline $\begin{array}{l}\text { VOLUME } \\
\text { CURIES }\end{array}$ & $\begin{array}{r}49 \\
533062\end{array}$ & $\begin{array}{r}<1 \\
49612\end{array}$ & $\begin{array}{r}<1 \\
1569\end{array}$ & & & $\begin{array}{r}<1 \\
794\end{array}$ & $\begin{array}{l}<1 \\
<1\end{array}$ & $\begin{array}{r}<1 \\
5148\end{array}$ & $\begin{array}{r}<1 \\
1045\end{array}$ & $\begin{array}{r}<1 \\
124\end{array}$ & $\begin{array}{l}<1 \\
<1\end{array}$ & $\begin{array}{r}53 \\
591,354\end{array}$ \\
\hline \multicolumn{13}{|l|}{ INTEC } \\
\hline $\begin{array}{l}\text { VOLUME } \\
\text { CURIES }\end{array}$ & & $\begin{array}{r}2 \\
<1\end{array}$ & $\begin{array}{l}7 \\
<1\end{array}$ & $\begin{array}{l}17 \\
<1\end{array}$ & $\begin{array}{r}159 \\
4\end{array}$ & $\begin{array}{r}112 \\
5\end{array}$ & $\begin{array}{r}702 \\
56\end{array}$ & $\begin{array}{r}142 \\
14\end{array}$ & $\begin{array}{l}26 \\
15\end{array}$ & $\begin{array}{r}209 \\
4\end{array}$ & $\begin{array}{r}972 \\
11\end{array}$ & $\begin{array}{r}2,347 \\
109\end{array}$ \\
\hline \multicolumn{13}{|l|}{ NRF } \\
\hline $\begin{array}{l}\text { VOLUME } \\
\text { CURIES }\end{array}$ & & & & & & & & & & & $\begin{array}{r}5 \\
<1\end{array}$ & $\begin{array}{l}5 \\
0\end{array}$ \\
\hline \multicolumn{13}{|l|}{ PBF } \\
\hline $\begin{array}{l}\text { VOLUME } \\
\text { CURIES }\end{array}$ & $\begin{array}{r}6 \\
<1\end{array}$ & $\begin{array}{l}23 \\
<1\end{array}$ & $\begin{array}{l}21 \\
<1\end{array}$ & $\begin{array}{l}24 \\
<1\end{array}$ & $\begin{array}{l}10 \\
<1\end{array}$ & $\begin{array}{r}7 \\
<1\end{array}$ & $\begin{array}{r}6 \\
<1\end{array}$ & $\begin{array}{l}26 \\
<1\end{array}$ & $\begin{array}{r}48 \\
1\end{array}$ & $\begin{array}{l}38 \\
10\end{array}$ & $\begin{array}{l}49 \\
30\end{array}$ & $\begin{array}{r}258 \\
42\end{array}$ \\
\hline \multicolumn{13}{|l|}{ SMC } \\
\hline $\begin{array}{l}\text { VOLUME } \\
\text { CURIES }\end{array}$ & & & & & & & & & $\begin{array}{l}<1 \\
<1\end{array}$ & & & $\begin{array}{l}0 \\
0\end{array}$ \\
\hline \multicolumn{13}{|l|}{ TAN } \\
\hline $\begin{array}{l}\text { VOLUME } \\
\text { CURIES }\end{array}$ & & & & & $<1$ & & $\begin{array}{l}25 \\
<1\end{array}$ & $\begin{array}{l}<1 \\
<1\end{array}$ & $\begin{array}{l}13 \\
<1\end{array}$ & $\begin{array}{r}531 \\
15\end{array}$ & $\begin{array}{r}45 \\
1\end{array}$ & $\begin{array}{r}617 \\
16\end{array}$ \\
\hline \multicolumn{13}{|l|}{ TRA } \\
\hline VOLUME & & & $<1$ & & & $<1$ & & & & & $<1$ & 0 \\
\hline CURIES & & & $<1$ & & & $<1$ & & & & & $<1$ & 0 \\
\hline \multicolumn{13}{|l|}{ WROC } \\
\hline $\begin{array}{l}\text { VOLUME } \\
\text { CURIES }\end{array}$ & & & & & & & & & & & $\begin{array}{l}14 \\
<1\end{array}$ & $\begin{array}{r}14 \\
0\end{array}$ \\
\hline $\begin{array}{c}\text { TOTAL VOLUME } \\
\text { CURIES }\end{array}$ & $\begin{array}{r}55 \\
533062\end{array}$ & $\begin{array}{r}25 \\
49813\end{array}$ & $\begin{array}{r}29 \\
1569\end{array}$ & $\begin{array}{l}41 \\
<1\end{array}$ & $\begin{array}{r}171 \\
4\end{array}$ & $\begin{array}{l}120 \\
799\end{array}$ & $\begin{array}{r}734 \\
56\end{array}$ & $\begin{array}{r}169 \\
5162\end{array}$ & $\begin{array}{r}87 \\
1061\end{array}$ & $\begin{array}{l}778 \\
153\end{array}$ & $\begin{array}{r}1087 \\
43\end{array}$ & $\begin{array}{r}3,295 \\
591,522 \\
\end{array}$ \\
\hline
\end{tabular}




\section{INEEL RECORD-TO-DATE SUMMARY STORED WASTE}

\section{ANLW 1989 - 1998 CONTAINERIZED STORED WASTE}

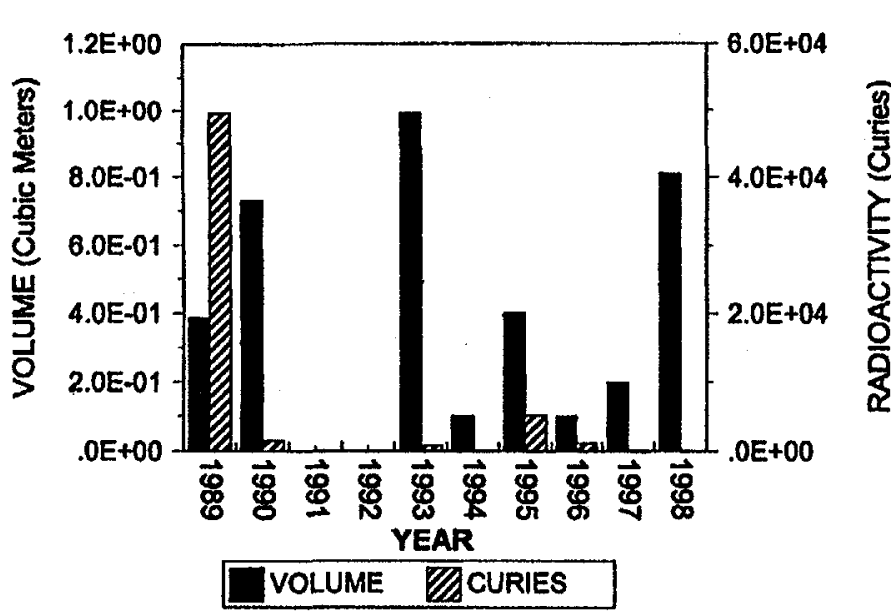

NRF 1989 - 1998 CONTAINERIZED STORED WASTE

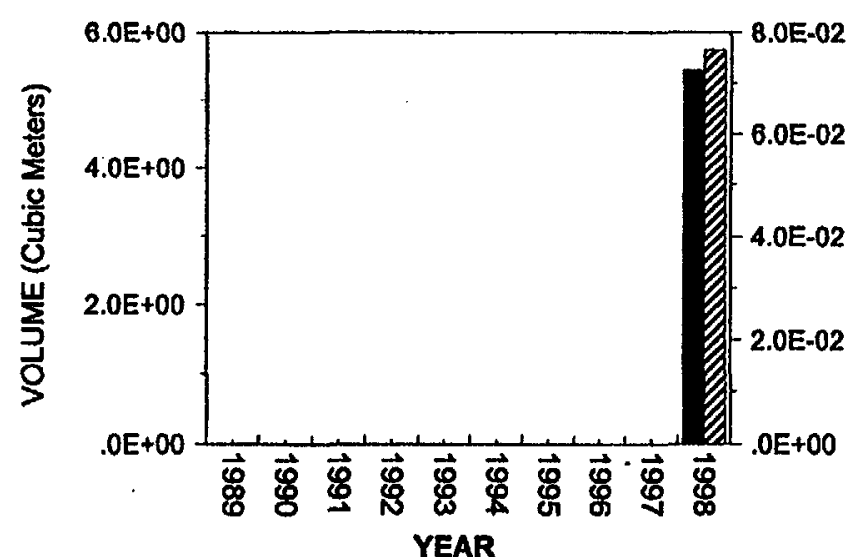

Graph: G228B01
INTEC 1989 - 1998 CONTAINERIZED STORED WASTE

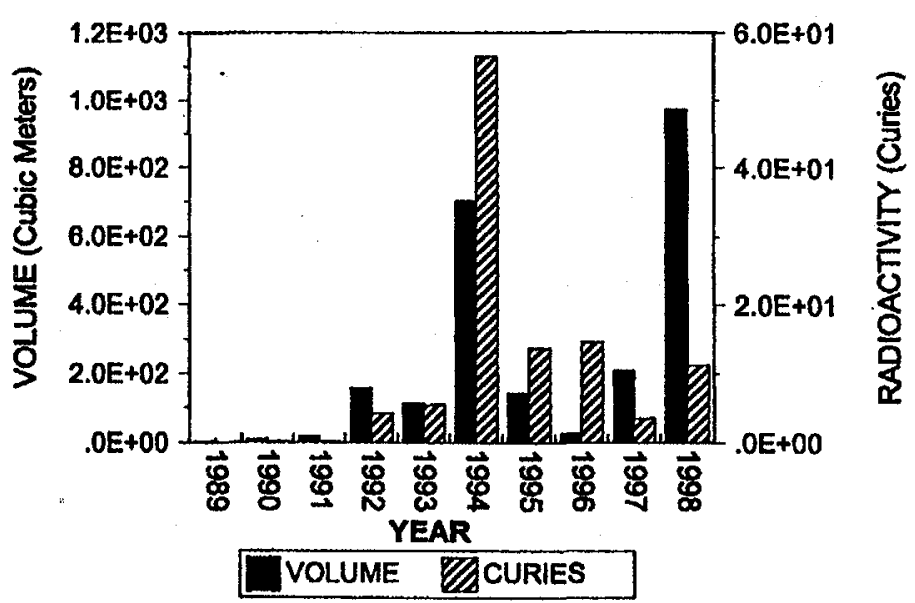

PBF 1989 - 1998 CONTAINERIZED STORED WASTE

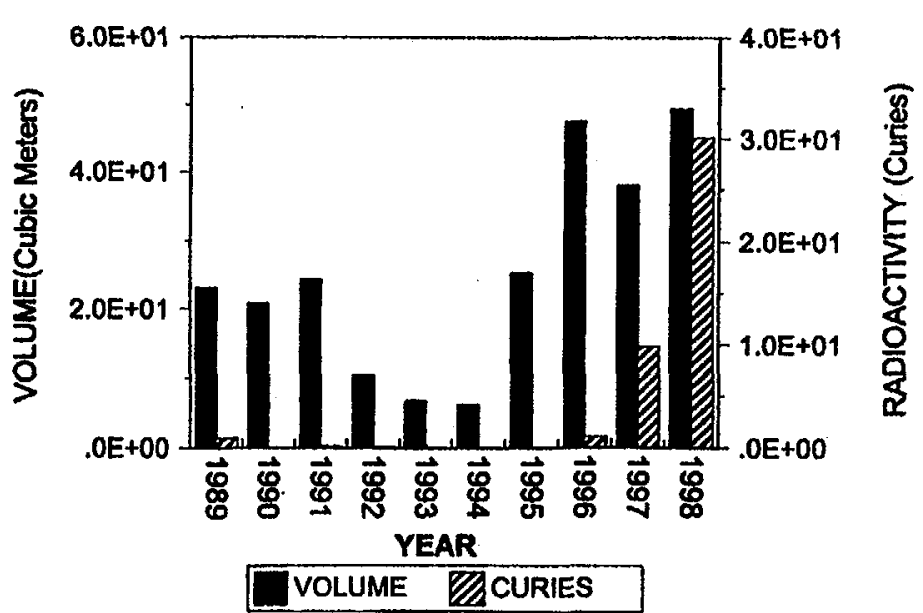




\section{INEEL RECORD-TO-DATE SUMMARY STORED WASTE}

SMC 1989 - 1998 CONTAINERIZED STORED WASTE

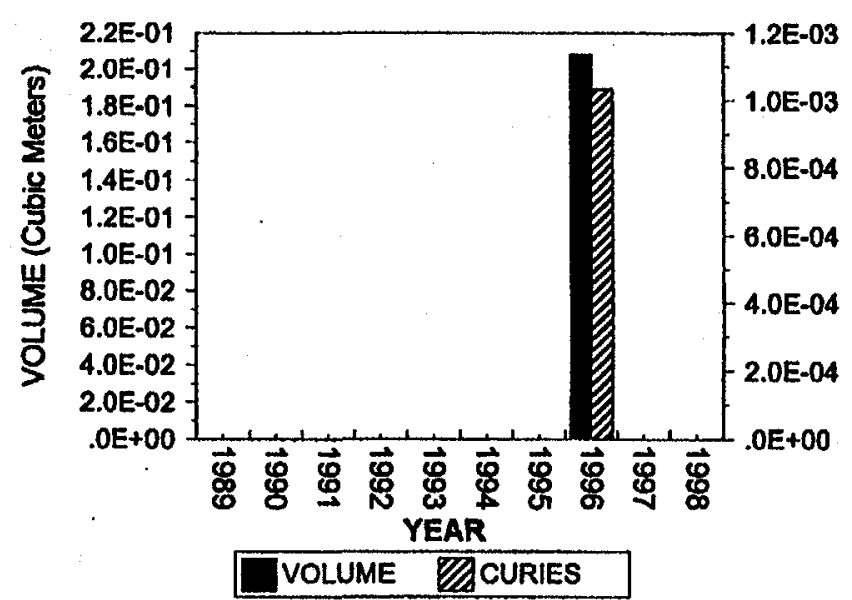

TRA 1989 - 1998 CONTAINERIZED STORED WASTE

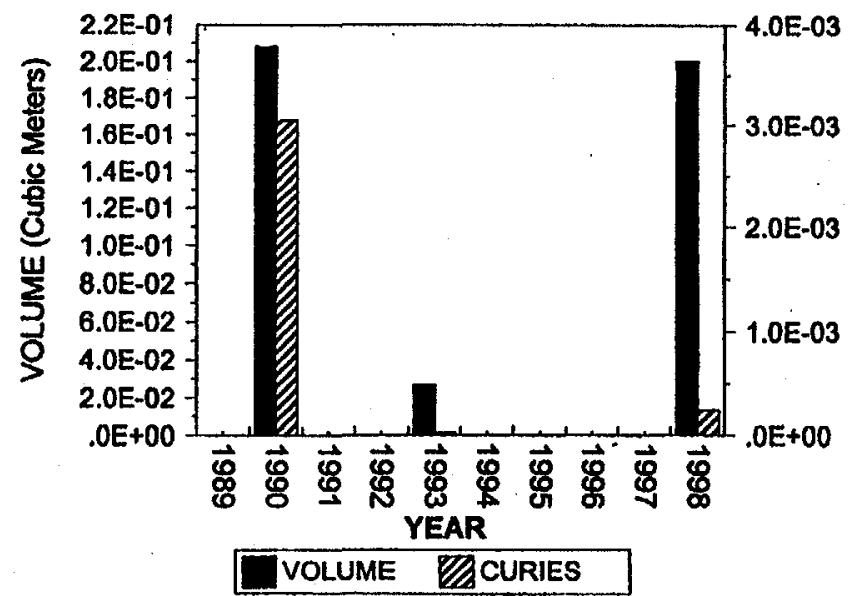

TAN 1989 - 1998 CONTAINERIZED STORED WASTE

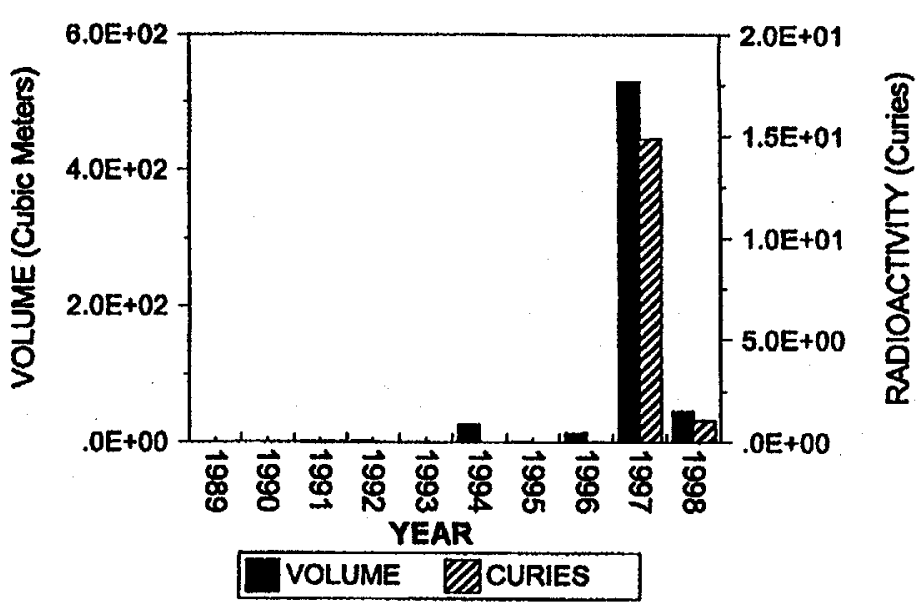

WROC 1989 - 1998 CONTAINERIZED STORED WASTE

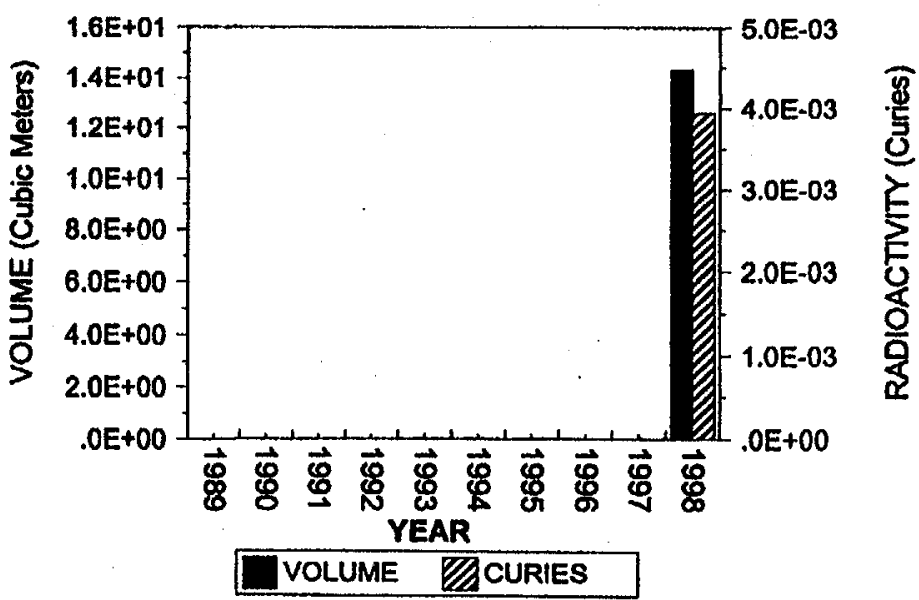




INEEL CONTAINERIZED RECCORD-TO-DATE SUMMARY
ILTSF WASTE



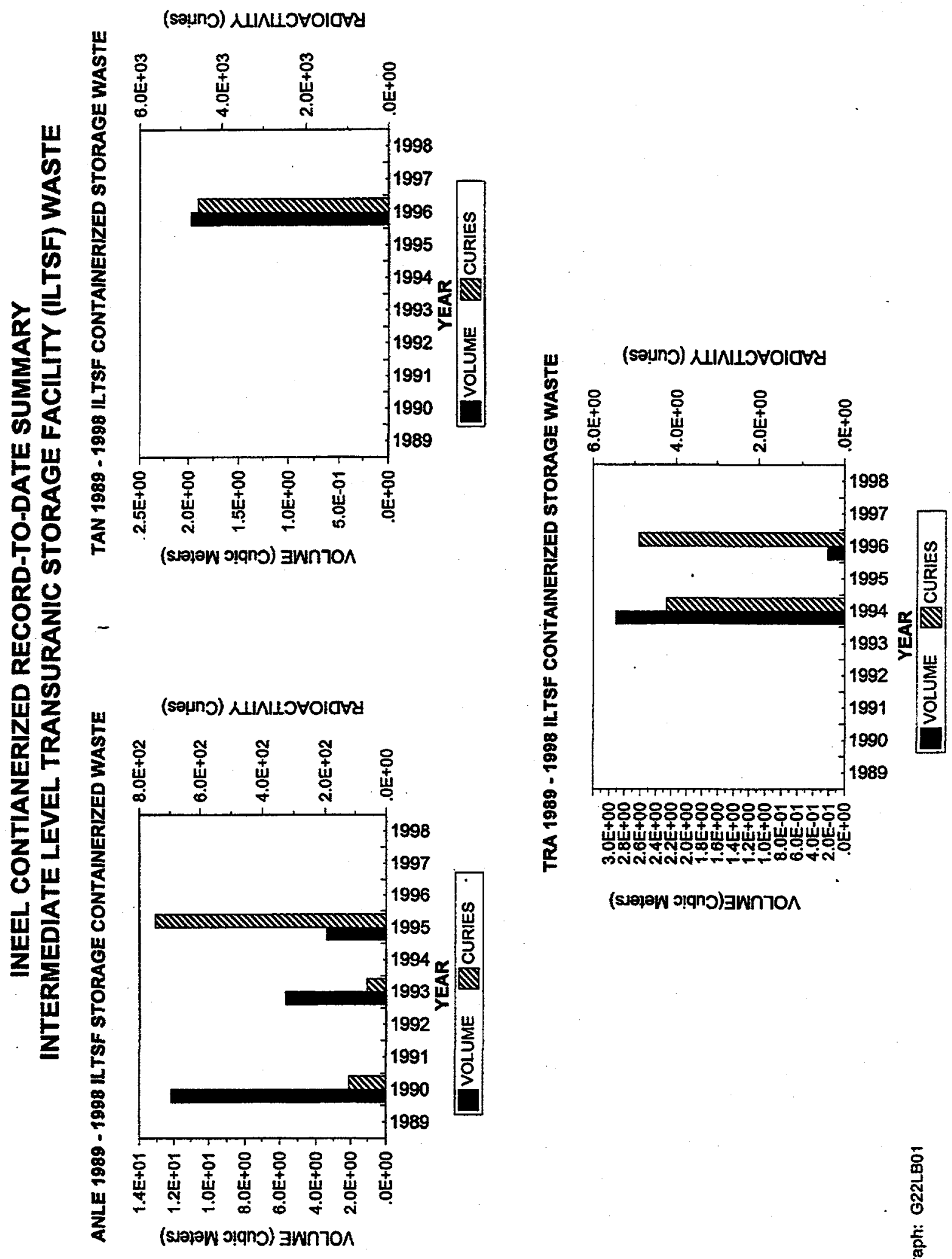


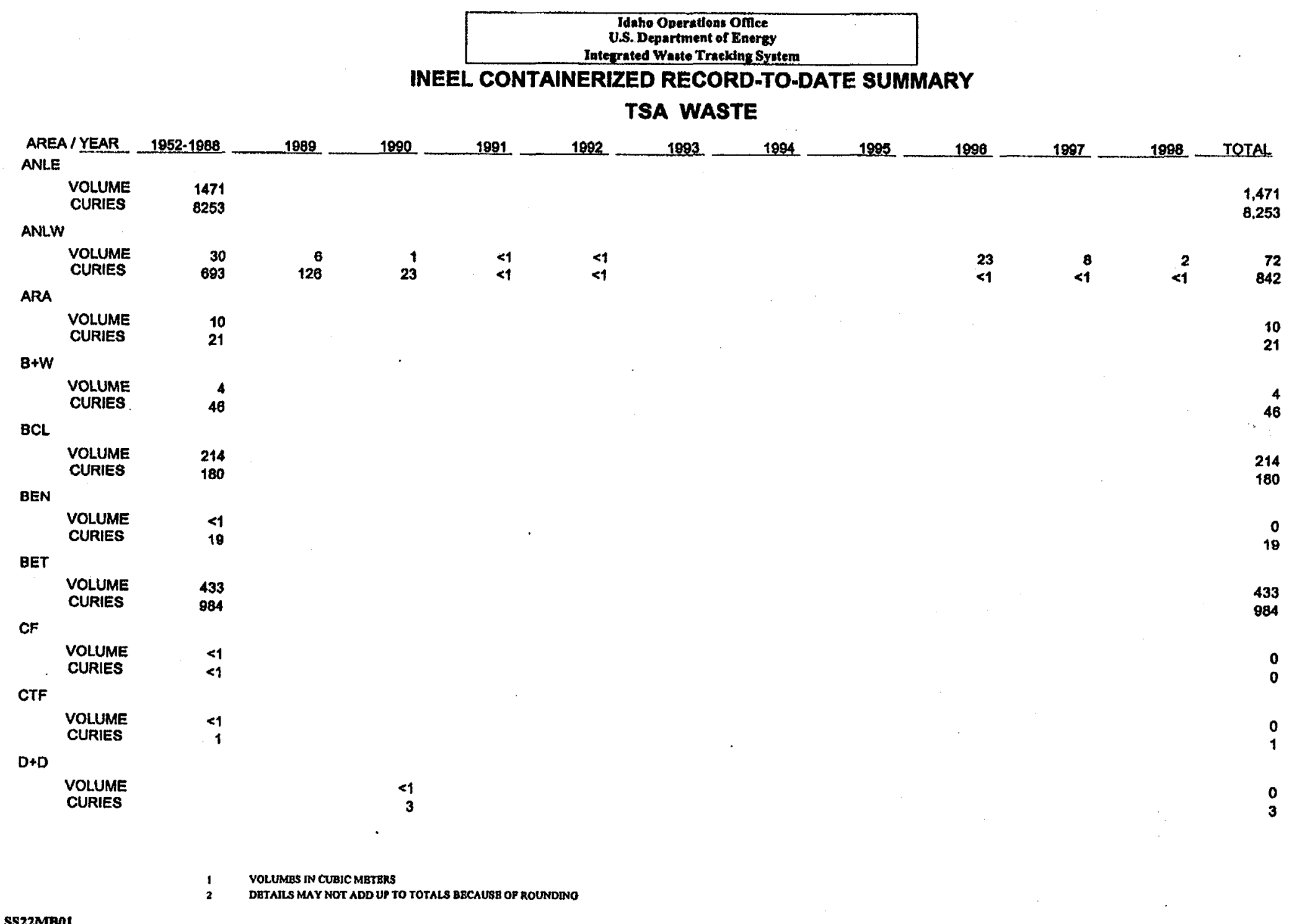




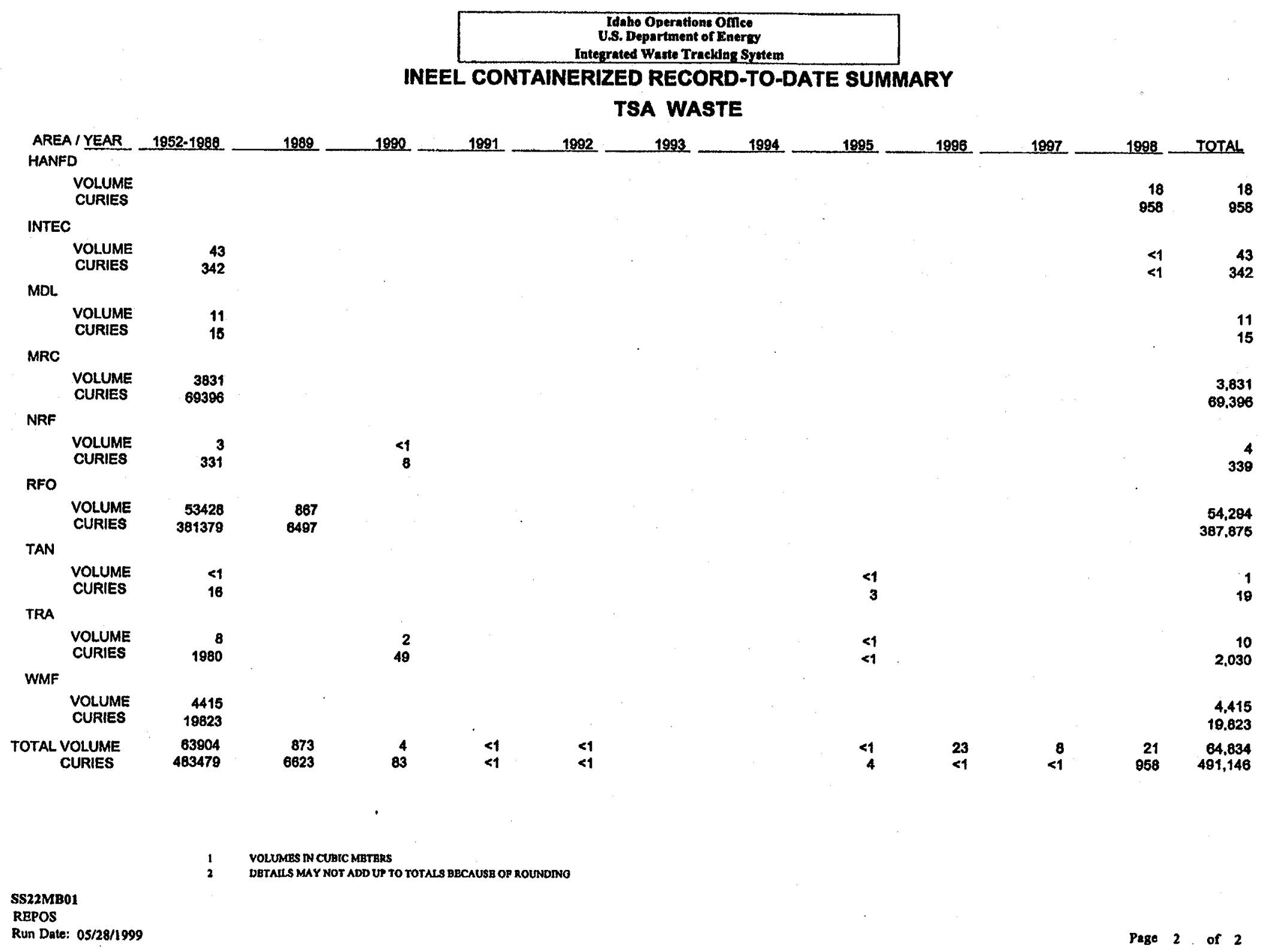



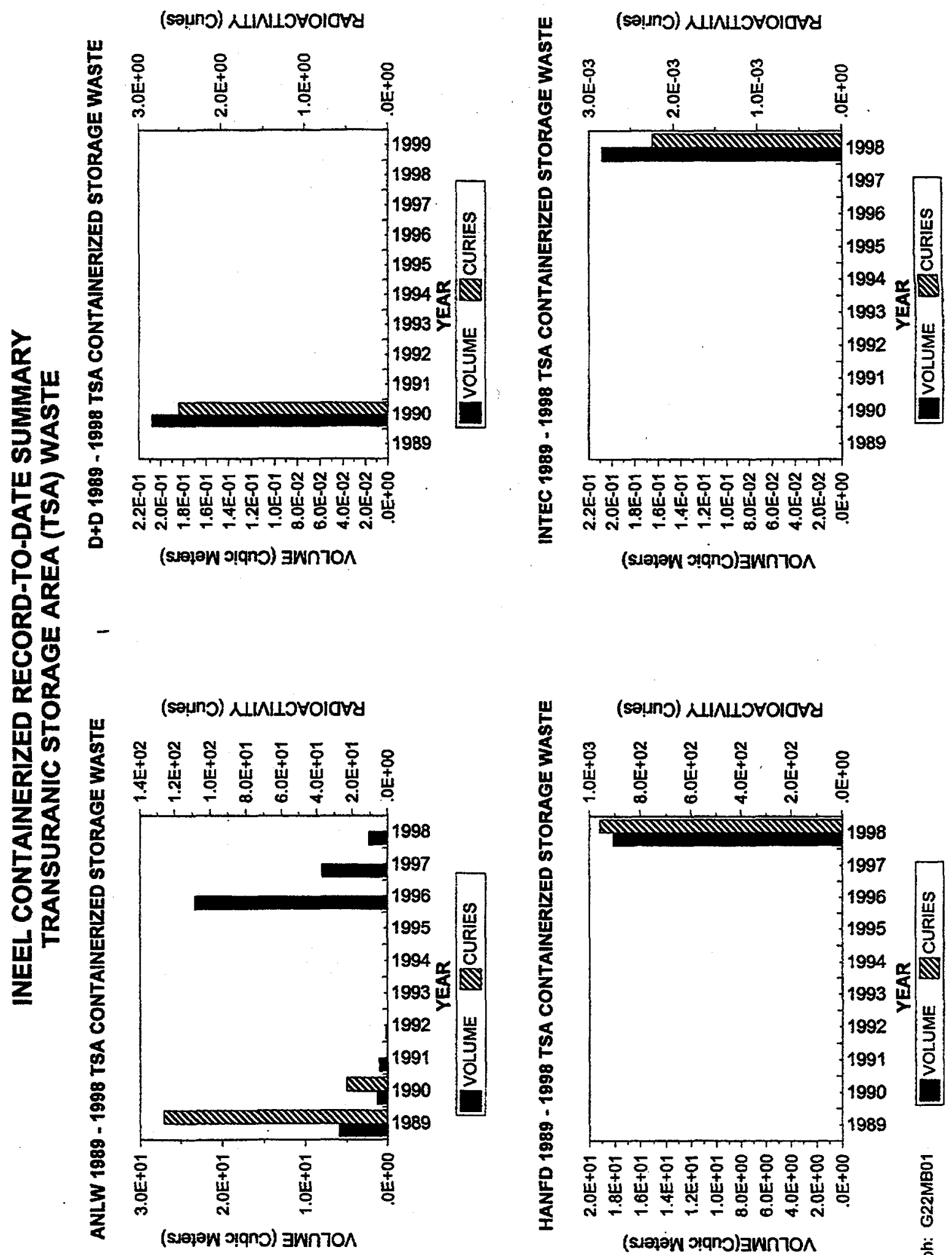

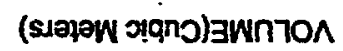

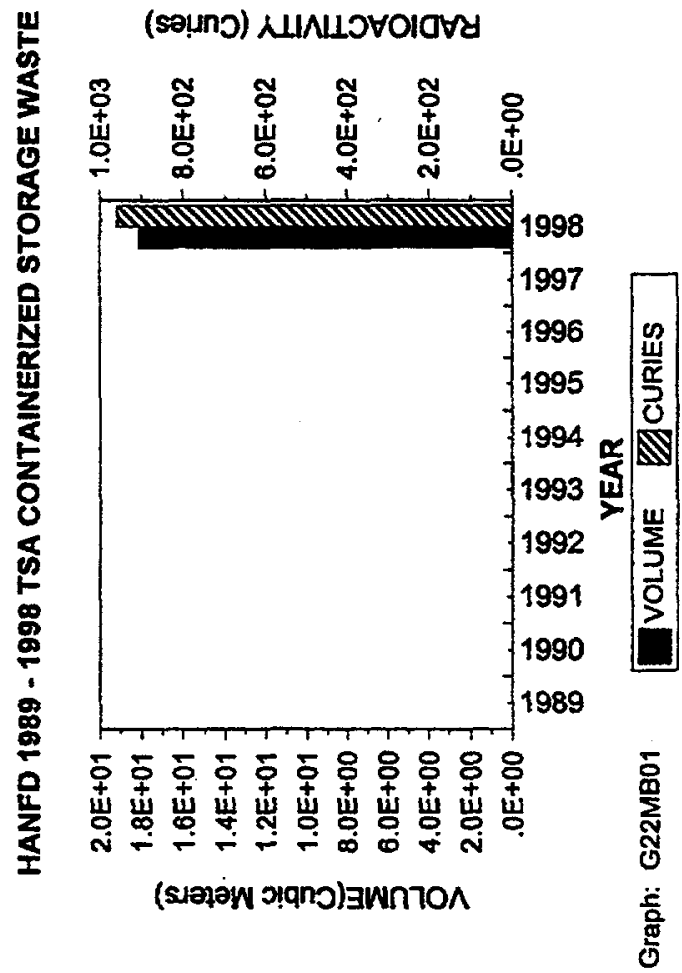



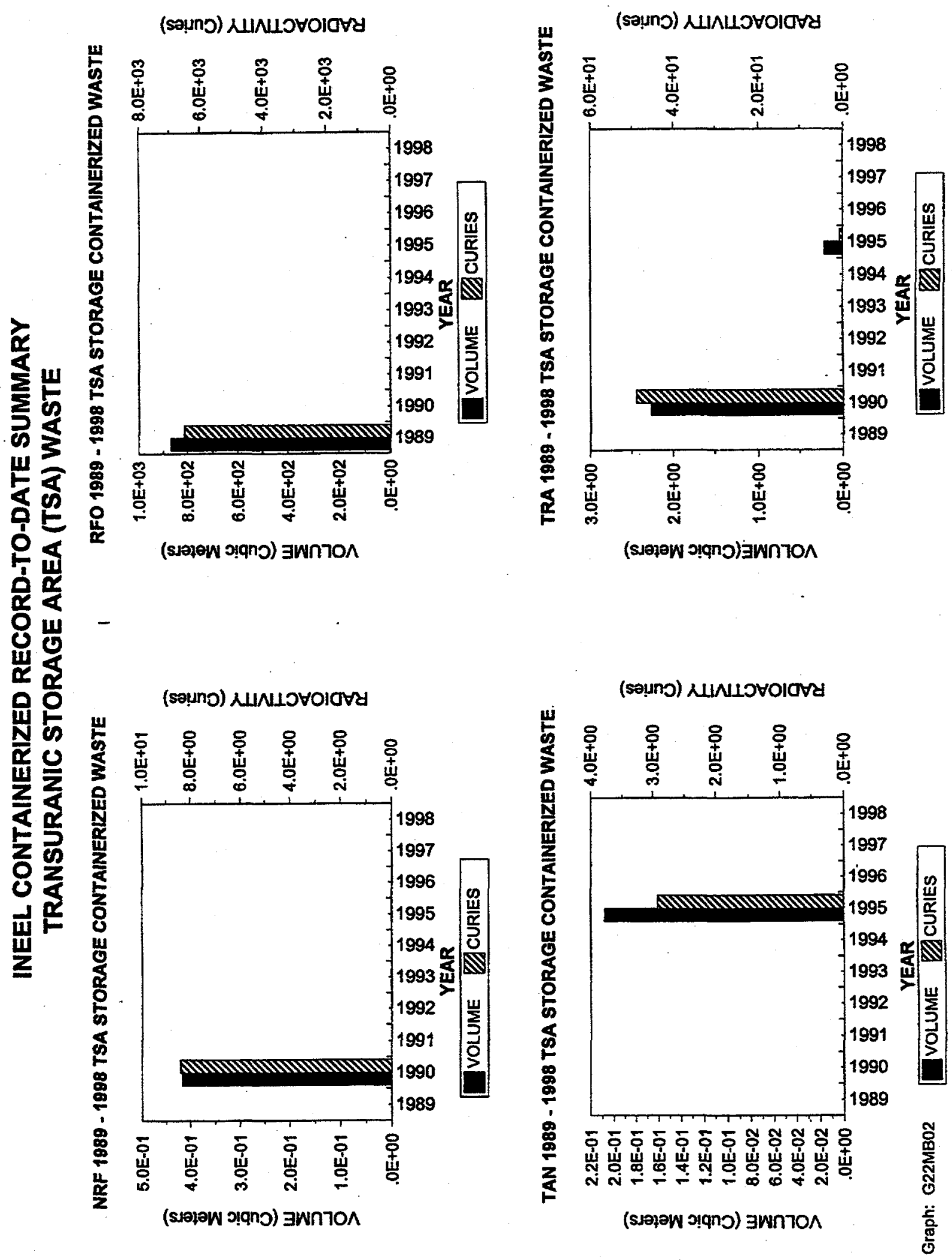


\section{Idaho Operations Once \\ INEEL CONTAINERIZED RECORD-TO-DATE SUMMARY}

\section{WERF WASTE}

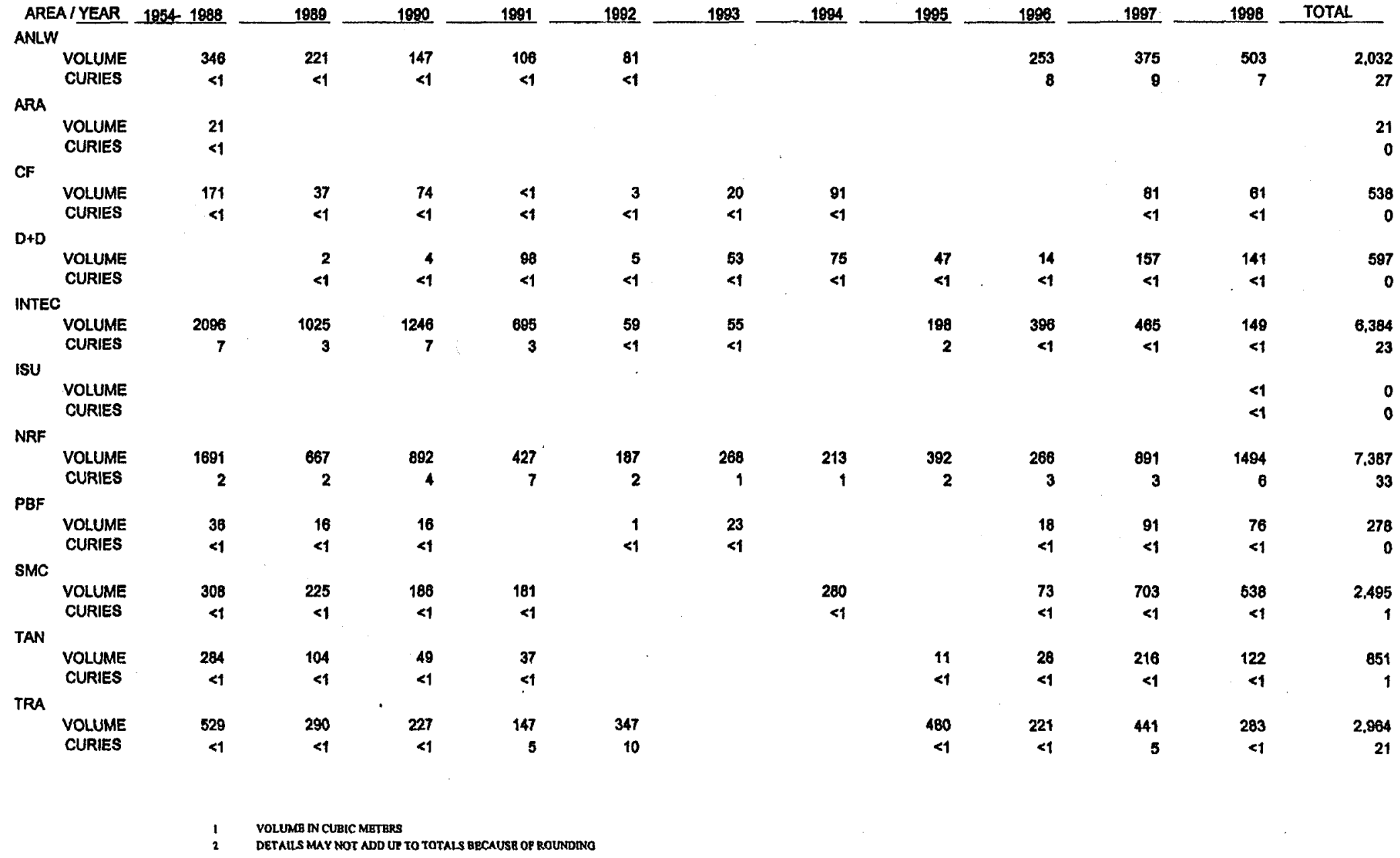




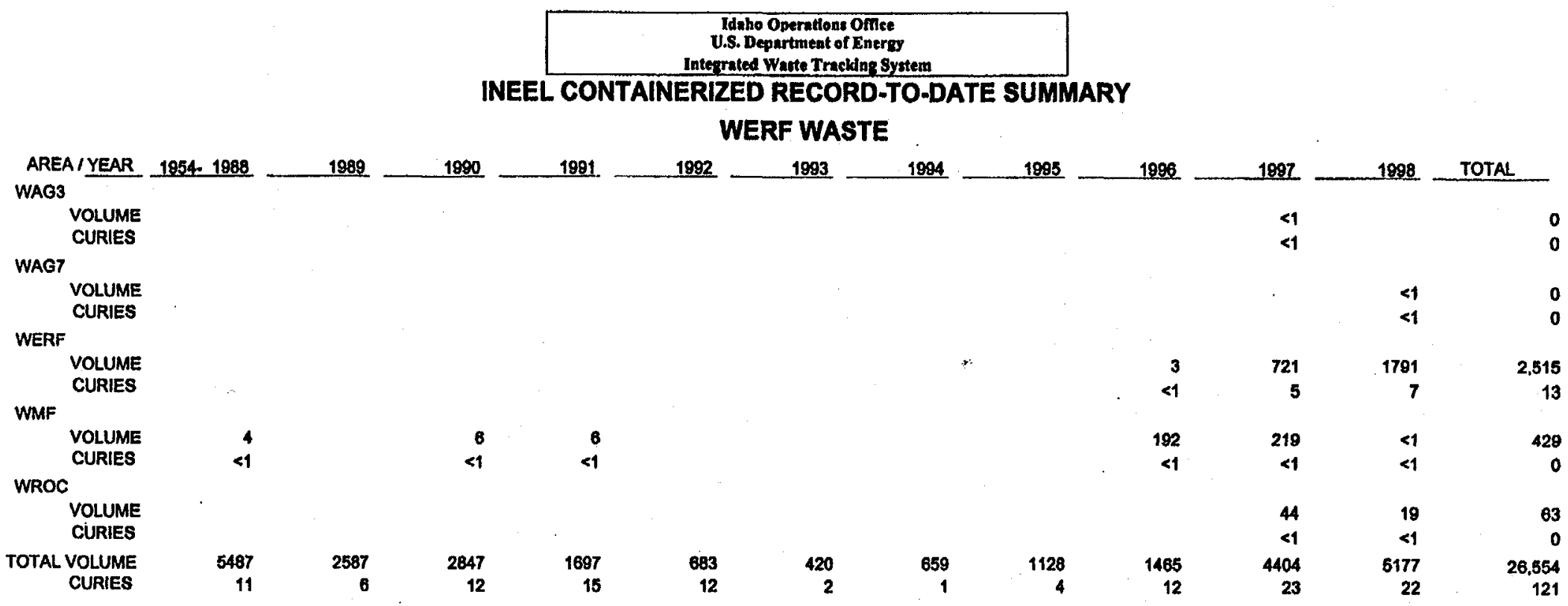




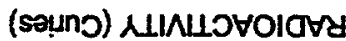
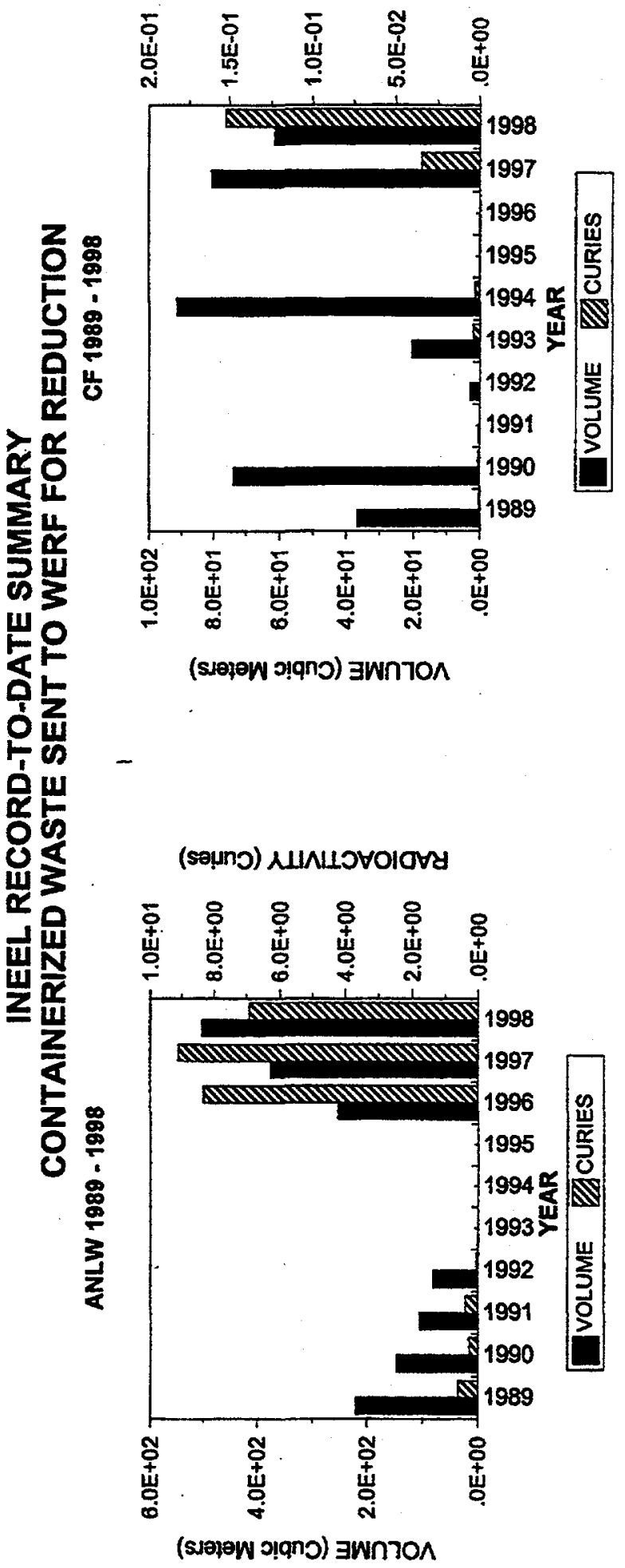

(soụno) NLINLOVOIGV8

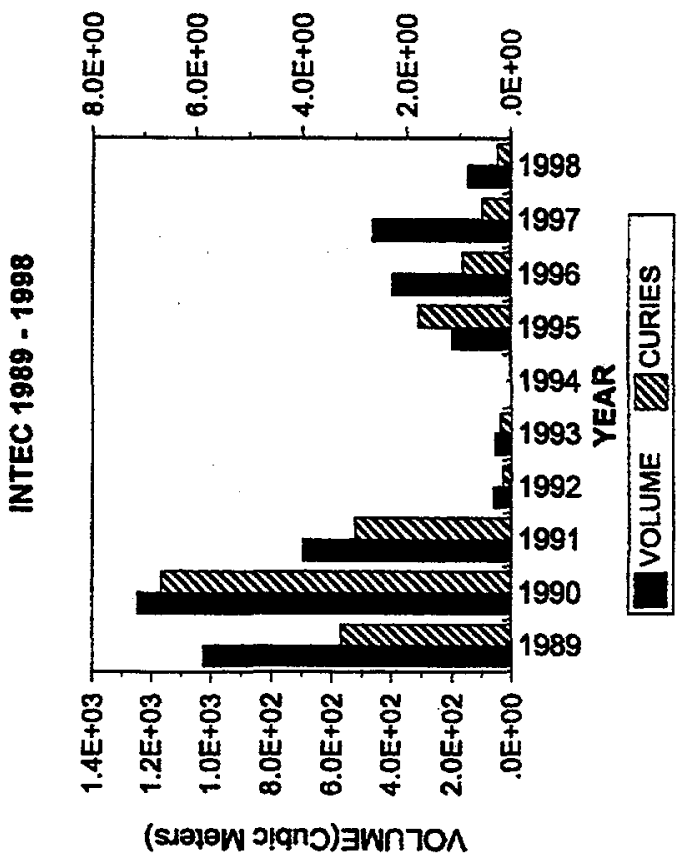

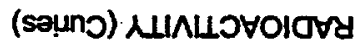

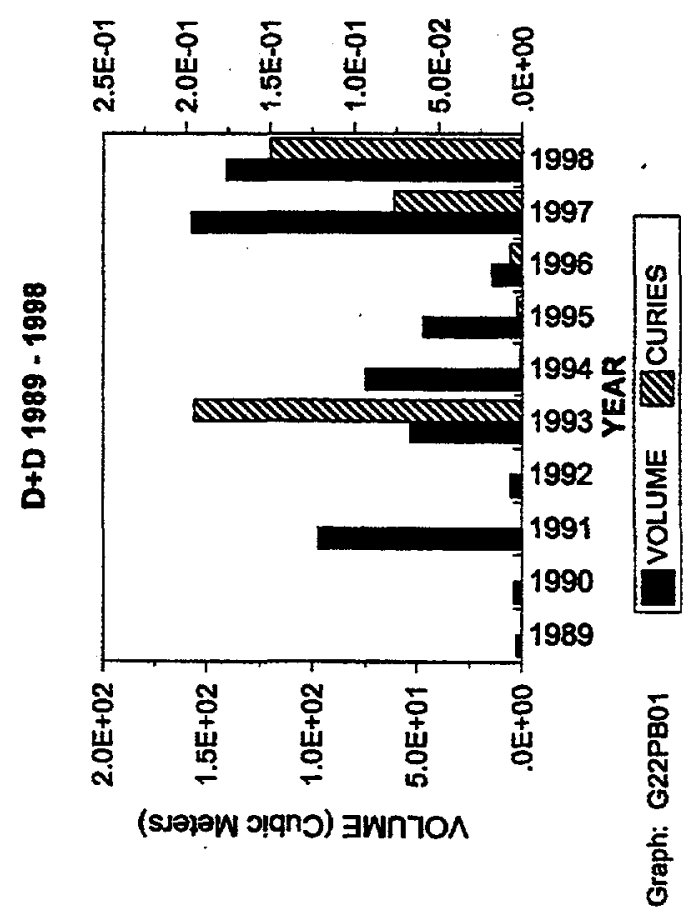




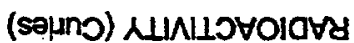

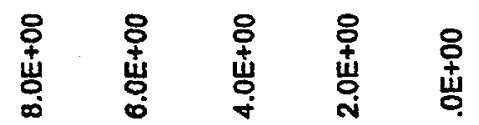

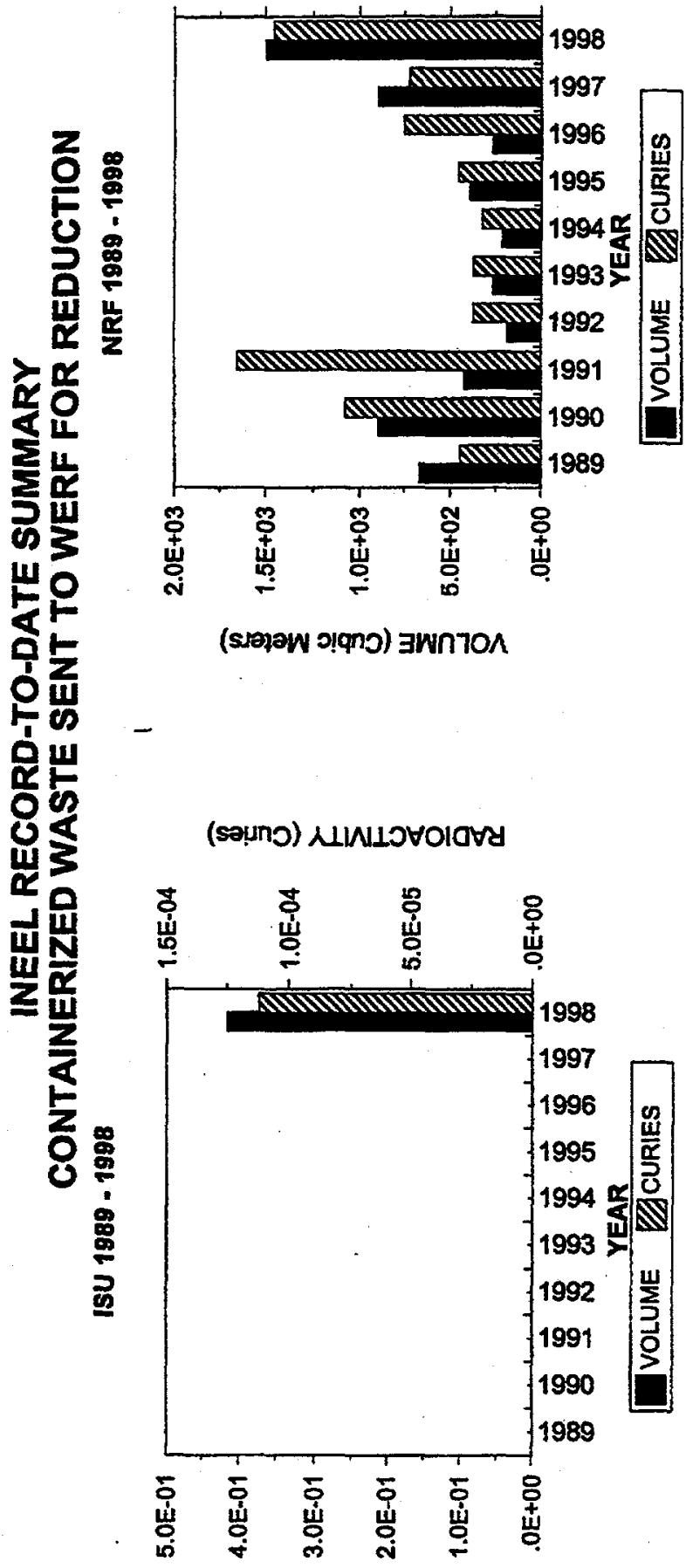

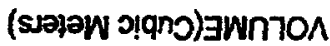

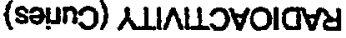

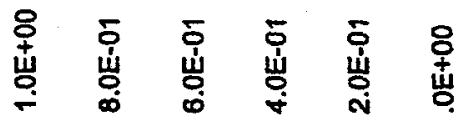

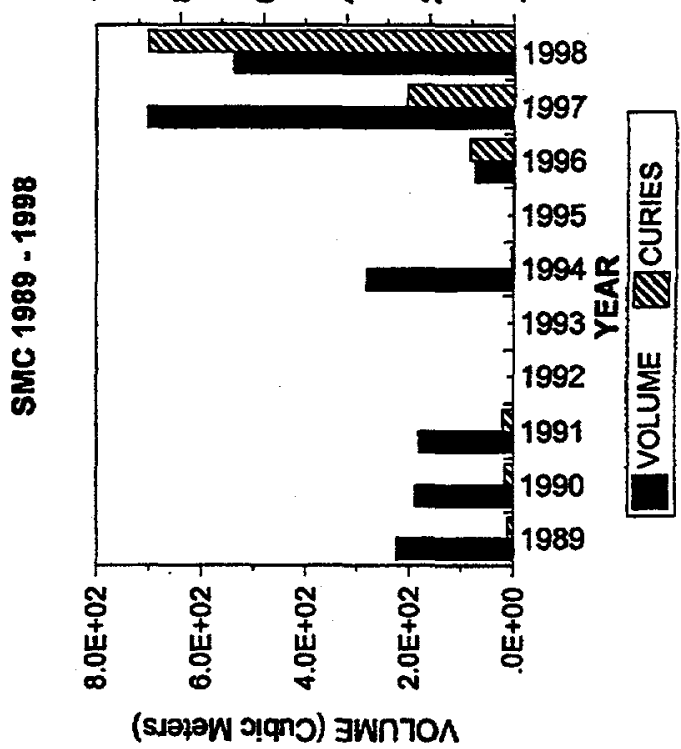

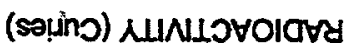

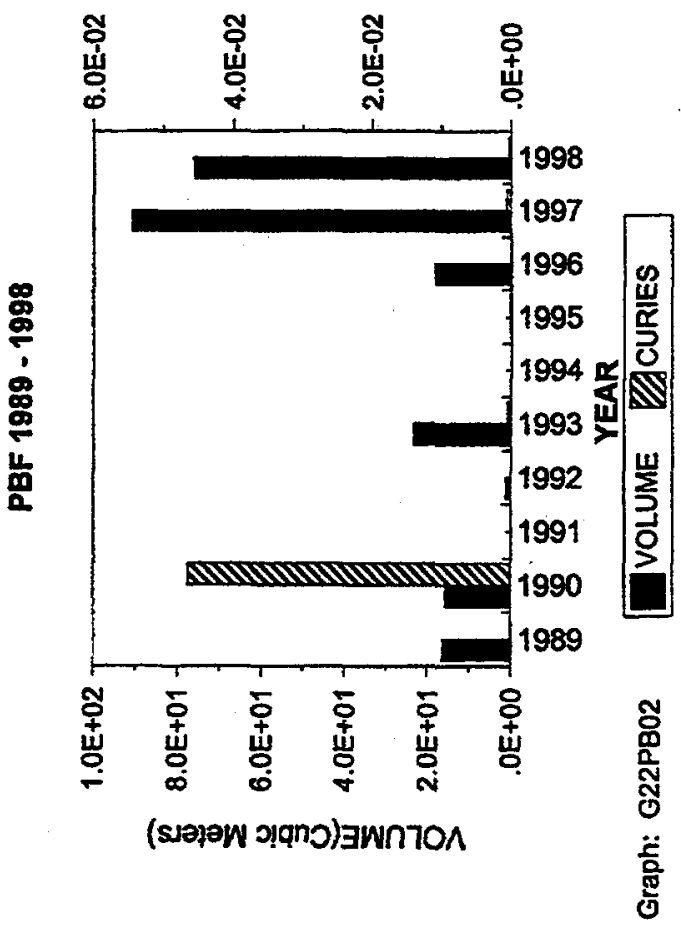


TAN $1989-1998$

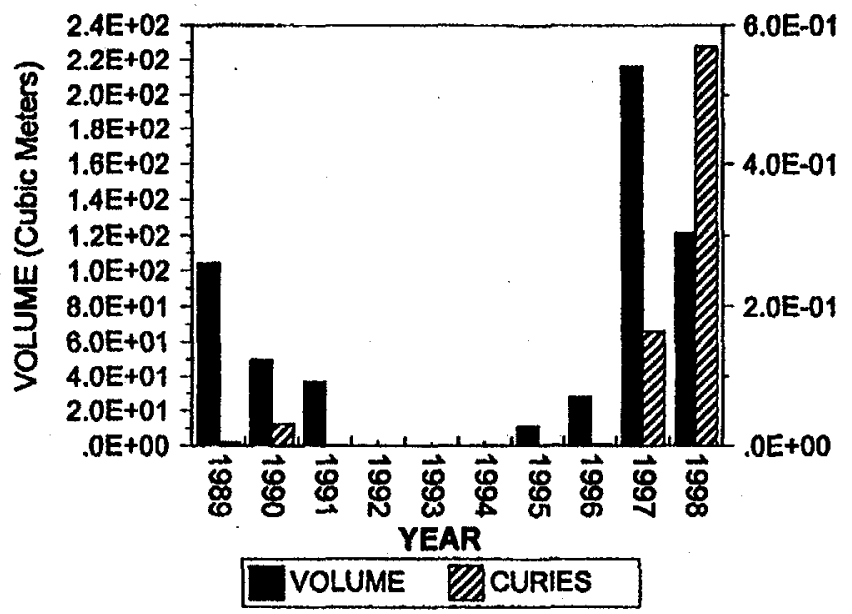

WAG3 1989 - 1998

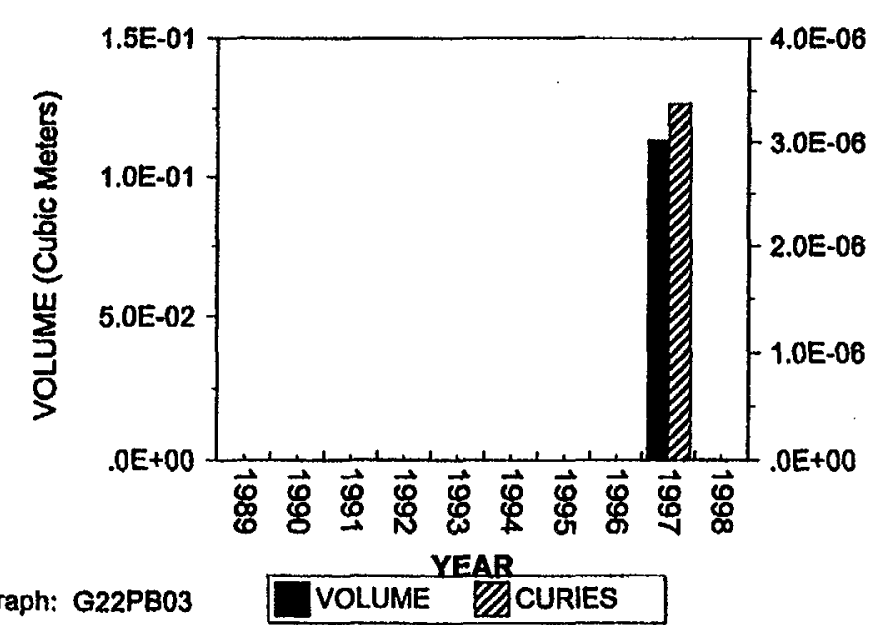

TRA $1989-1998$
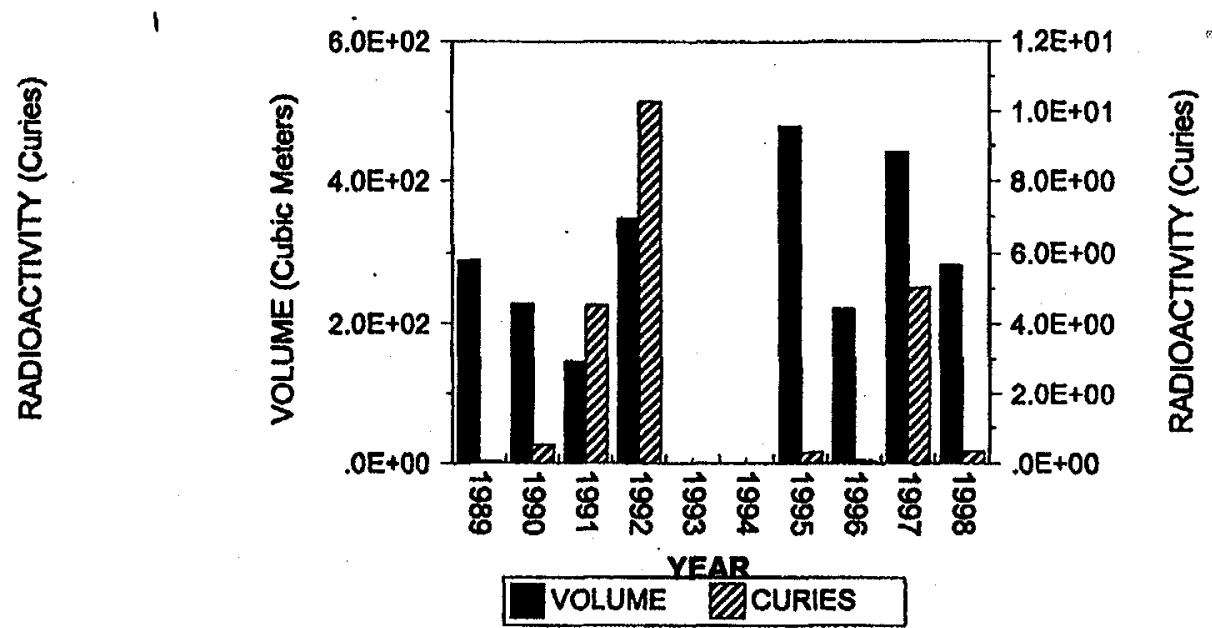

WAG7 1989 - 1998

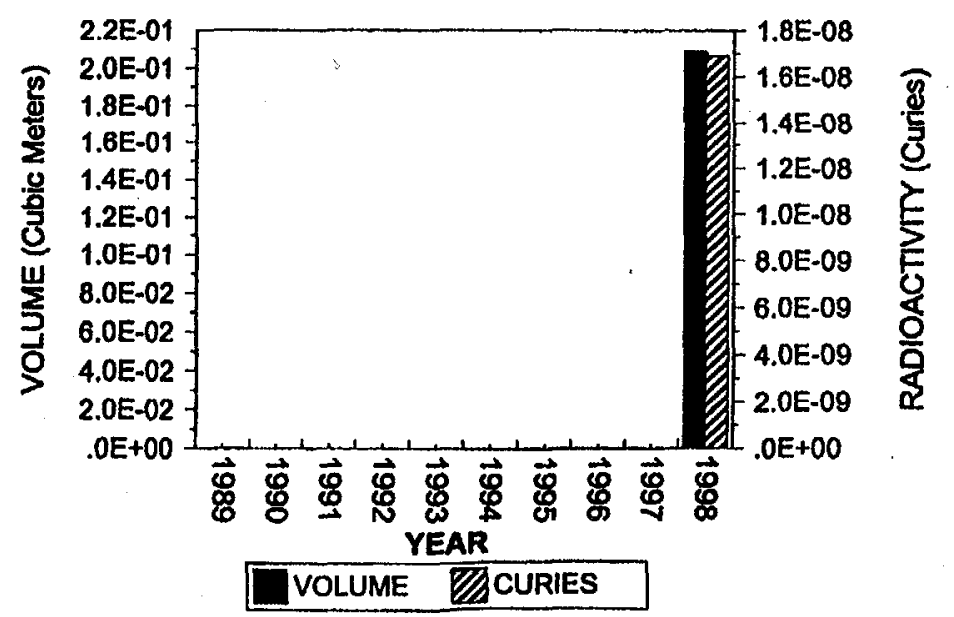



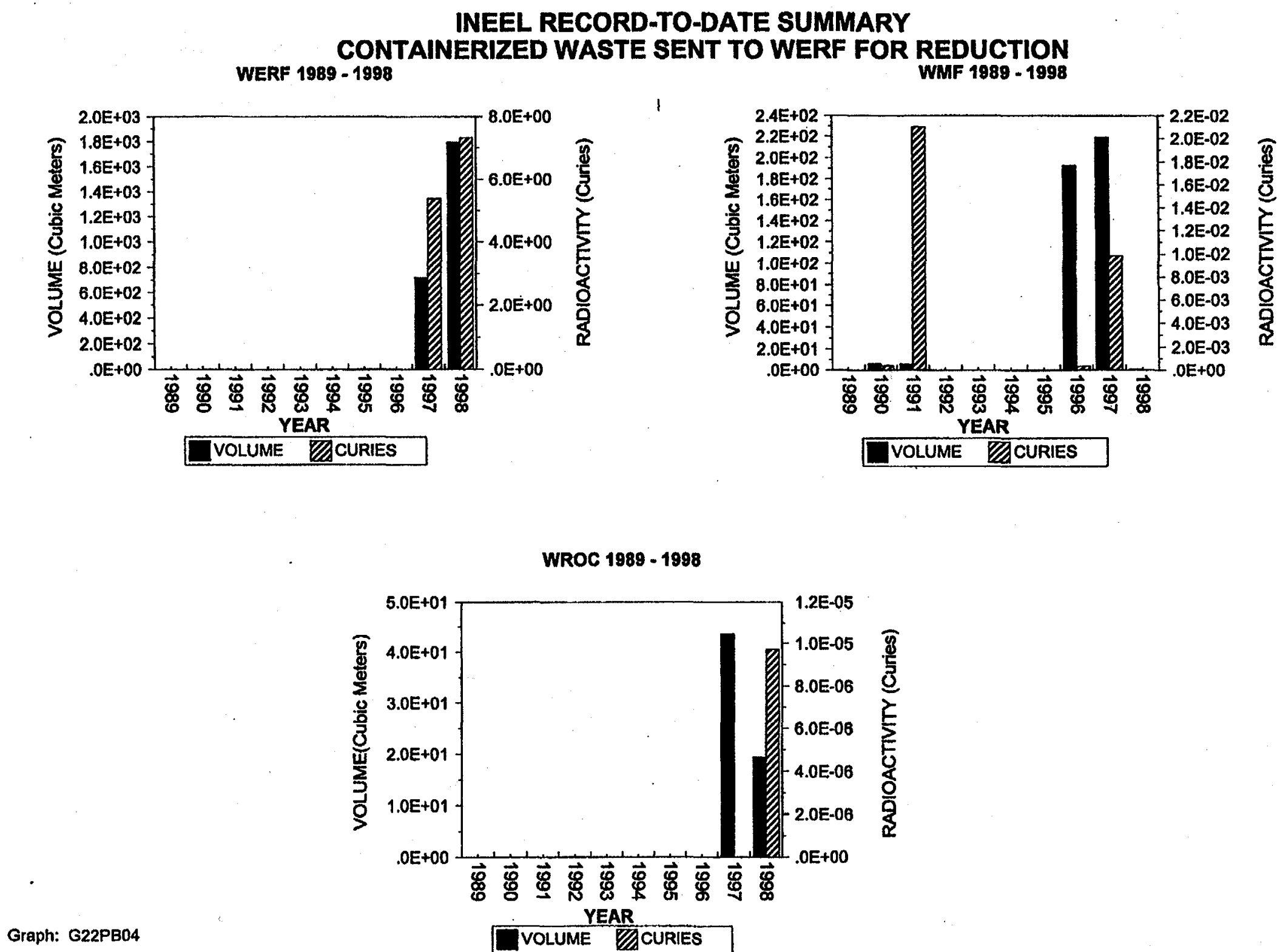
INEEL-40 
INEEL 1998 Year-to-Date Summary

INEEL Year To Date Summary, 1998.

INEEL-43

INEEL Year-To-Date Summary CY 1998 Pie Charts

INEEL-46

INEEL Year-To-Date CY 1998 Pie Charts

INEEL-47 


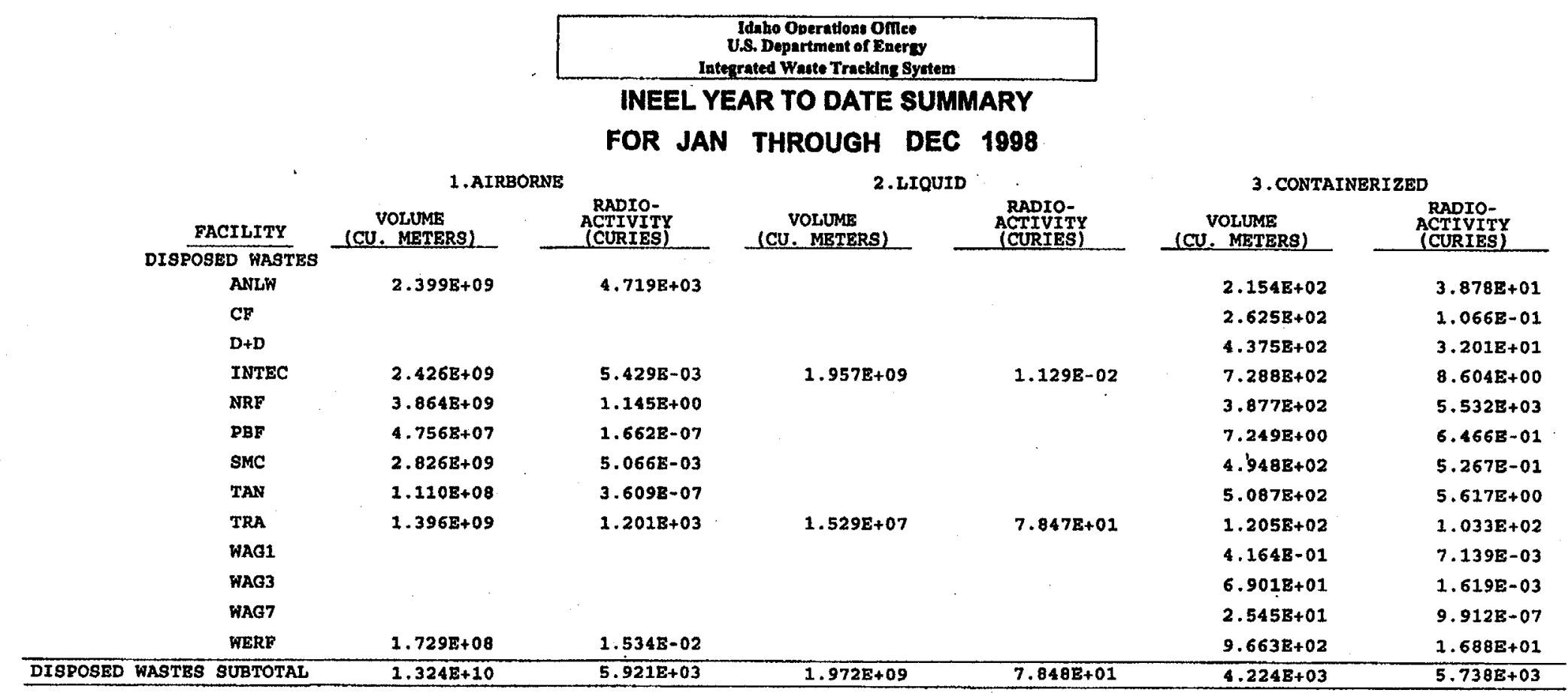



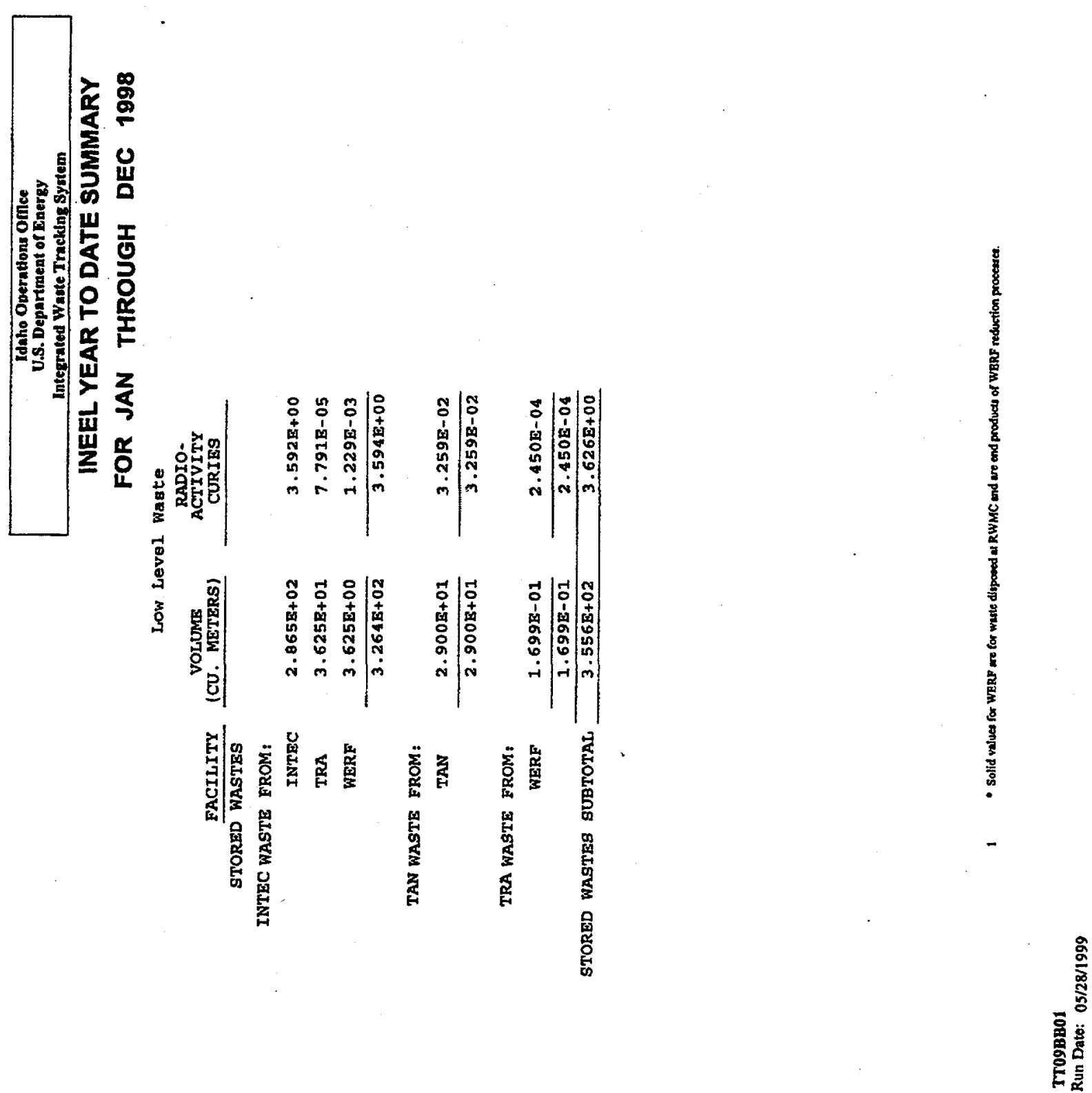


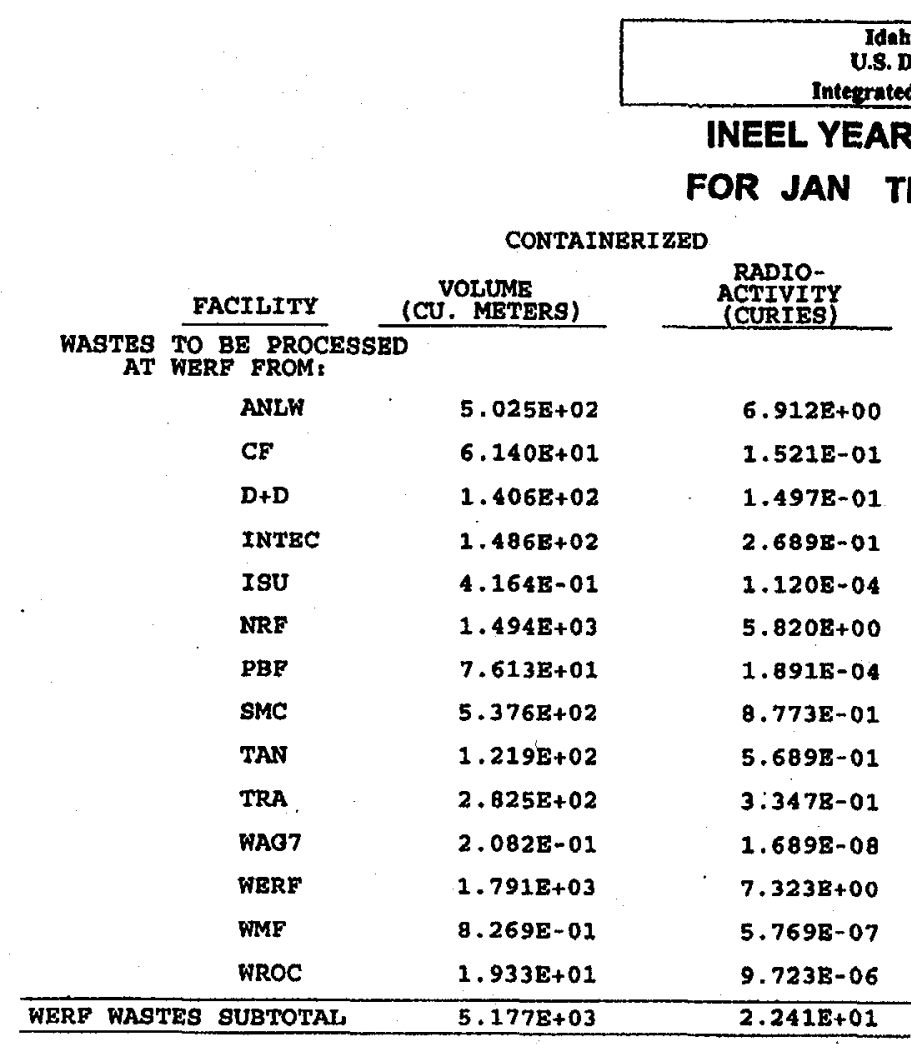

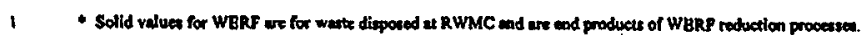




\section{INEEL YEAR-TO-DATE SUMMARY CY 1998}

INEEL AIRBORNE EMISSIONS

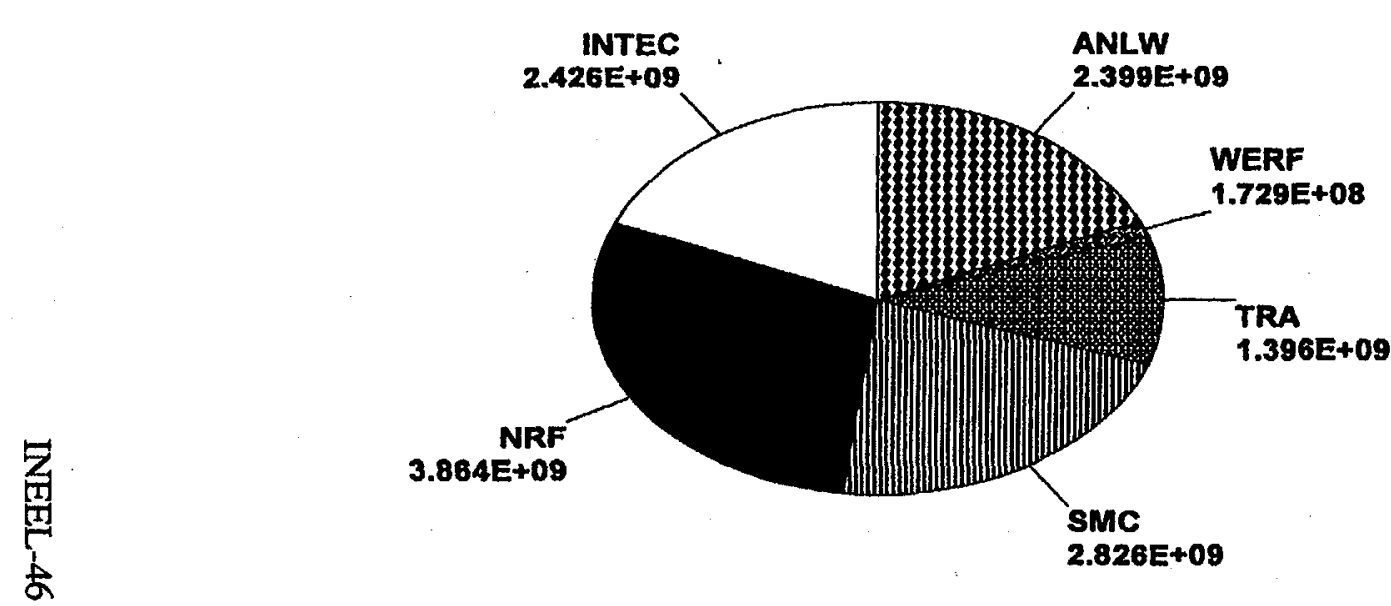

Volume (Cubic Meters) Areas not listed have values $<1 \%$ of the total

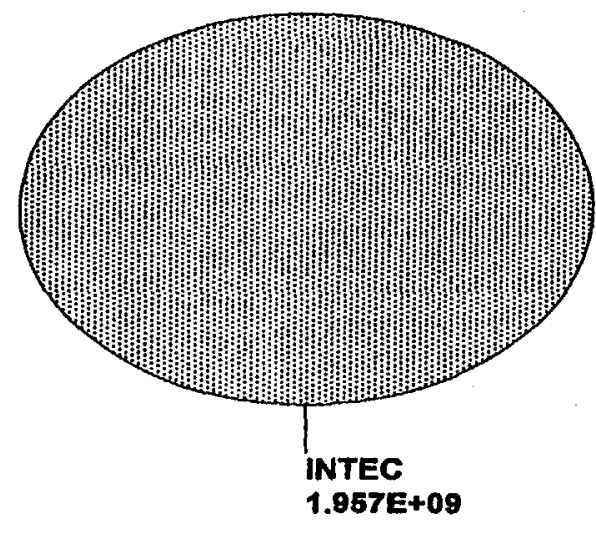

Volume (Liters)

Areas not listed have values $<1 \%$ of the total

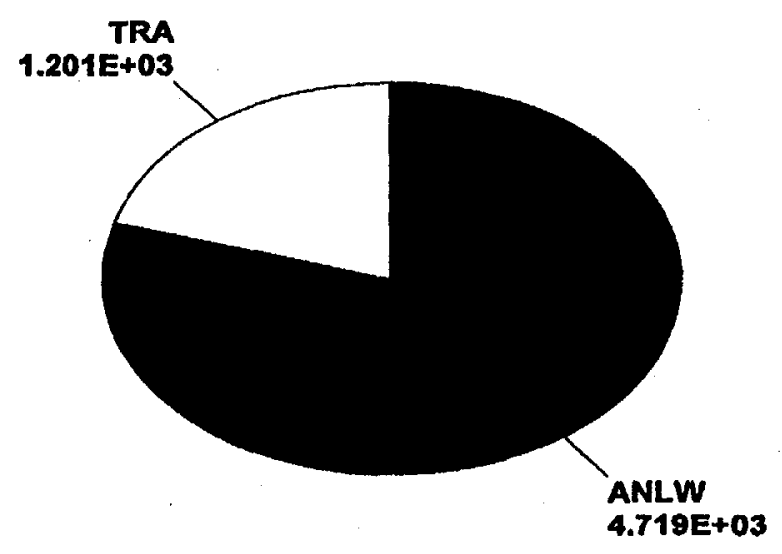

Radloactivity (Curles)

INEEL LIQUID EFFLUENT

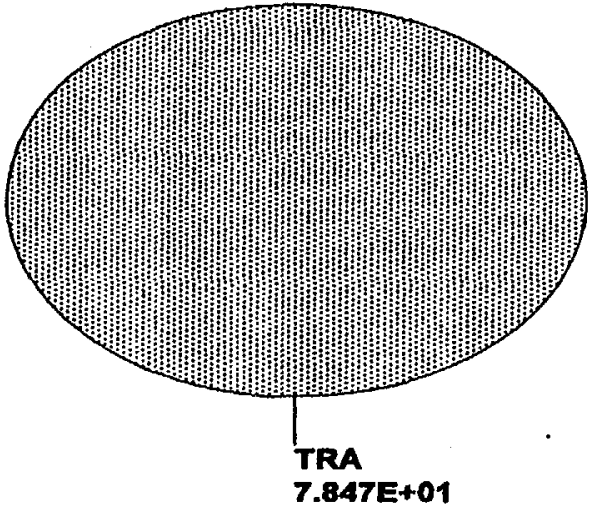

Radloactivity (Curles) 


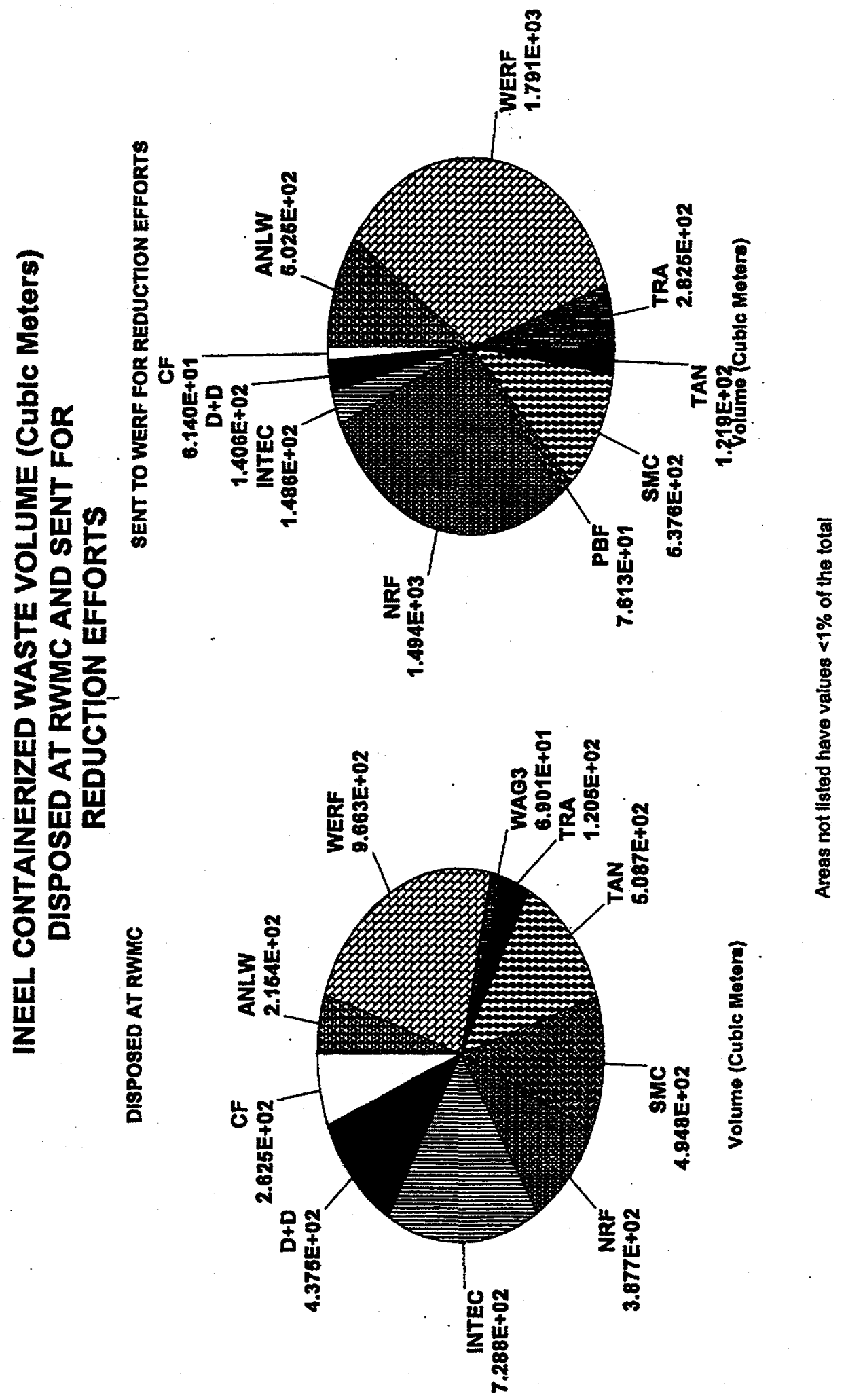

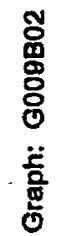




\section{Nuclide Summary in Curies for Airborne, Liquid, and Solid Waste}

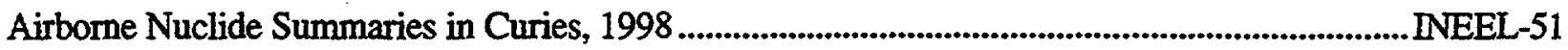

Liquid Nuclide Summary in Curies, 1998 ...............................................................................INEEL-55

Disposed Solid Waste Nuclide Summary in Curies, 1998 ........................................................INEEL-56

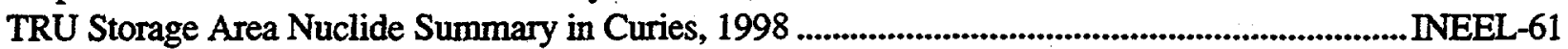

ANL-W Stored Storage Area Nuclide Summary in Curies, 1998 ...............................................INEEL-62

INTEC Stored Storage Area Nuclide Summary in Curies, 1998 ...............................................INEEL-63

TAN Stored Storage Area Nuclide Summary in Curies, 1998 ......................................................INEEL-66

TRA Stored Storage Area Nuclide Summary in Curies, 1998 .......................................................INEEL-67

WERF Stored Storage Area Nuclide Summary in Curies, 1998 .......................................................INEEL-68

Processed Nuclide Summary in Curies, 1998 INEEL-72 


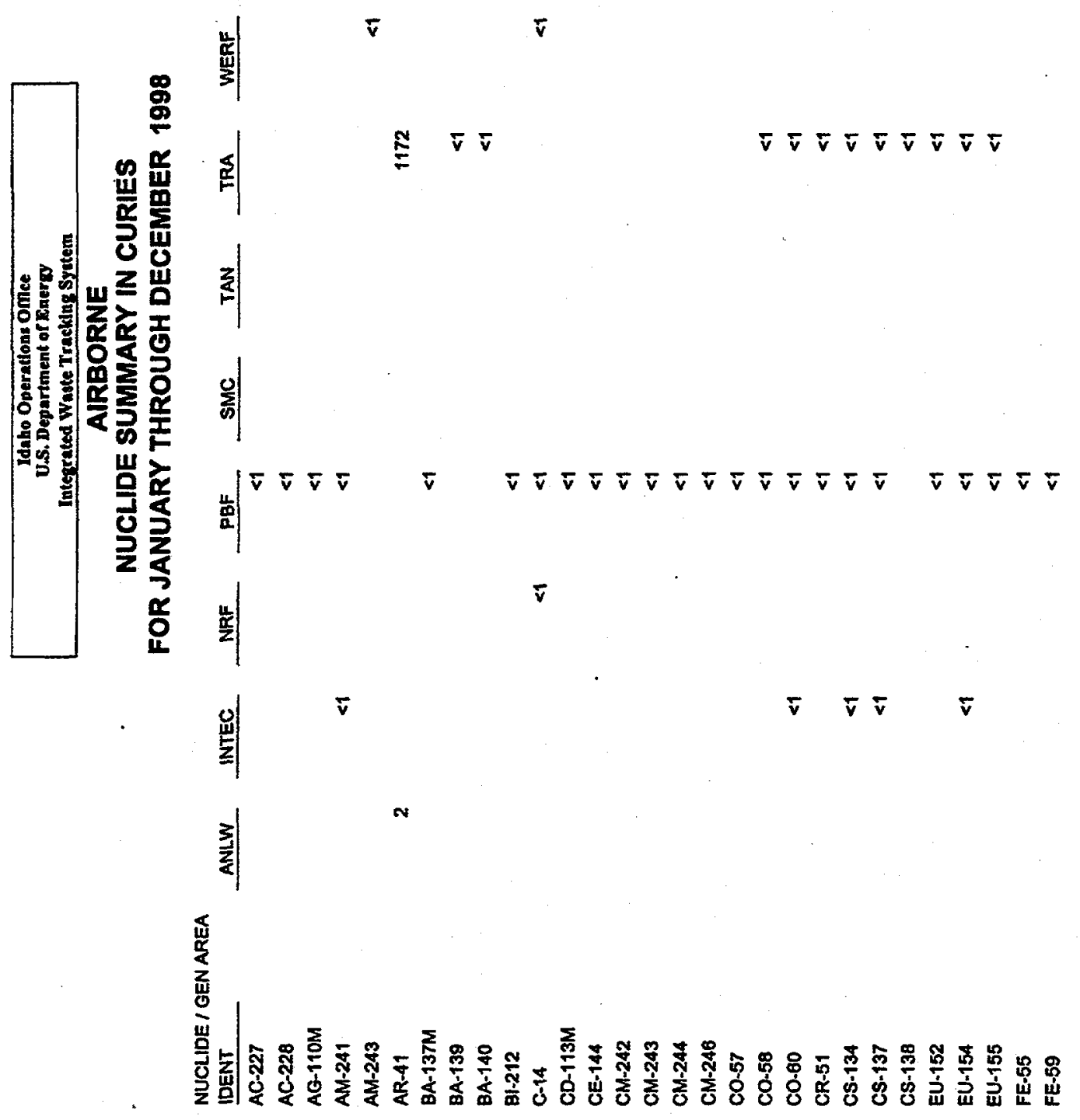




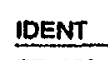

GD-153

GROS-BET-GAM

GROSS-ALPHA

GROSS-BETA

H-3

HF-175

HF-181

HG-203

$1-129$

I-131

$1-133$

I-135

$K-40$

KR-85

KR-85M

LA-140

MN-54

MN-56

NA-24

NB-94

NB-95

N159

NI-63

NP-237

PA-231

PA-233

PA-234

PA-234M

PB-212

Idaho Operation: Omce

U.s. Departmetion

AIRBORNE

NUCLIDE SUMMARY IN CURIES

FOR JANUARY THROUGH DECEMBER 1998

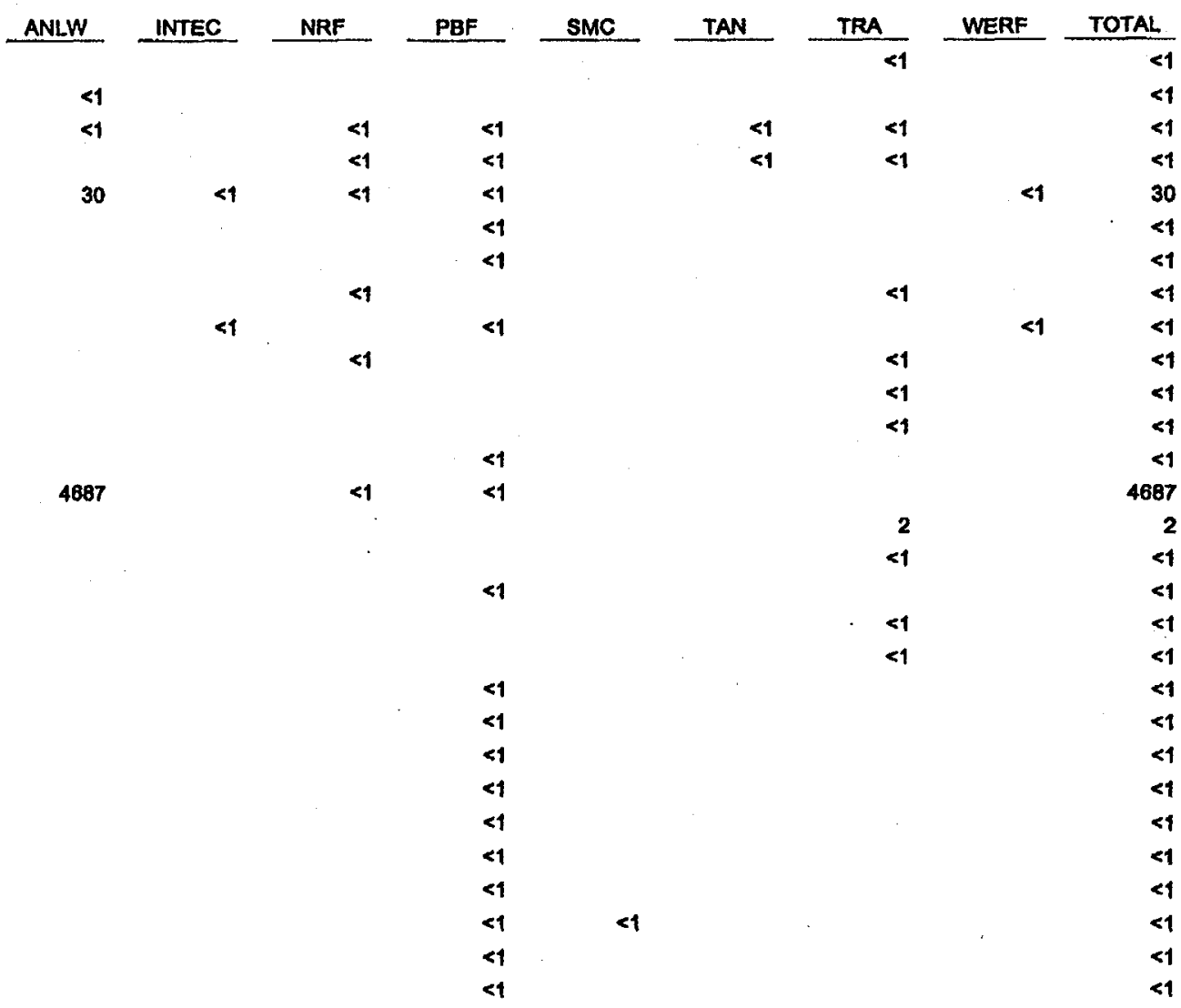




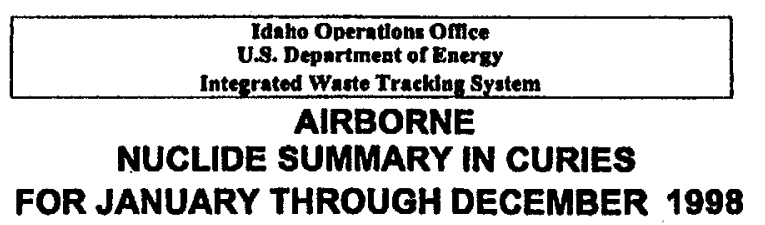

\begin{tabular}{|c|c|c|c|c|c|c|c|c|c|}
\hline IDENT & ANLW & INTEC & NRF & PBF & SMC & TAN & TRA & WERF & TOTAL \\
\hline PM-147 & & & & $<1$ & & & & & $<1$ \\
\hline PO-212 & & & & $<1$ & & & & & $<1$ \\
\hline$P O-216$ & & & & $<1$ & & & & & $<1$ \\
\hline PR-144 & & & & $<1$ & & & & & $<1$ \\
\hline PU-238 & & $<1$ & & $<1$ & & & & & $<1$ \\
\hline PU-239 & & $<1$ & & $<1$ & & & & $<1$ & $<1$ \\
\hline PU-240 & & & & $<1$ & & & & & $<1$ \\
\hline PU-241 & & & & $<1$ & & & & & $<1$ \\
\hline PU-242 & & & & $<1$ & & & & & $<1$ \\
\hline RA-224 & & & & eq & & & & & $<1$ \\
\hline RA-228 & & & & $<1$ & & & & & $<1$ \\
\hline RB-88 & & & & & & & 1 & & 1 \\
\hline RE-188 & & & & & & & $<1$ & & $<1$ \\
\hline RH-100 & & & & $<1$ & & & & & $<1$ \\
\hline RN-220 & & & & $<1$ & & & & & $<1$ \\
\hline RU-106 & & $<1$ & & $<1$ & & & & & $<1$ \\
\hline SB-124 & & & & $<1$ & & & & & $<1$ \\
\hline$S B: 125$ & & $<1$ & & $<1$ & & & & & $<1$ \\
\hline SE-75 & & & & $<1$ & & & & & $<1$ \\
\hline SM-151 & & & & $<1$ & & & & & $<1$ \\
\hline SN-113 & & & & $<1$ & & & & & $<1$ \\
\hline SR-90 & & $<1$ & & $<1$ & & & $<1$ & & $<1$ \\
\hline TA-182 & & & & $<1$ & & & & & $<1$ \\
\hline TC-99 & & & & $<1$ & & & & & $<1$ \\
\hline TC-89M & & & & & & & $<1$ & & $<1$ \\
\hline TH.228 & & & & $<1$ & & & & $<1$ & $<1$ \\
\hline TH-230 & & & & $<1$ & & & & $<1$ & $<1$ \\
\hline TH-231 & & & & $<1$ & & & & & $<1$ \\
\hline TH-232 & & & & $<1$ & & & & $<1$ & $<1$ \\
\hline
\end{tabular}

AE18AN01

REPOS

Run Date: 05/28/1999 


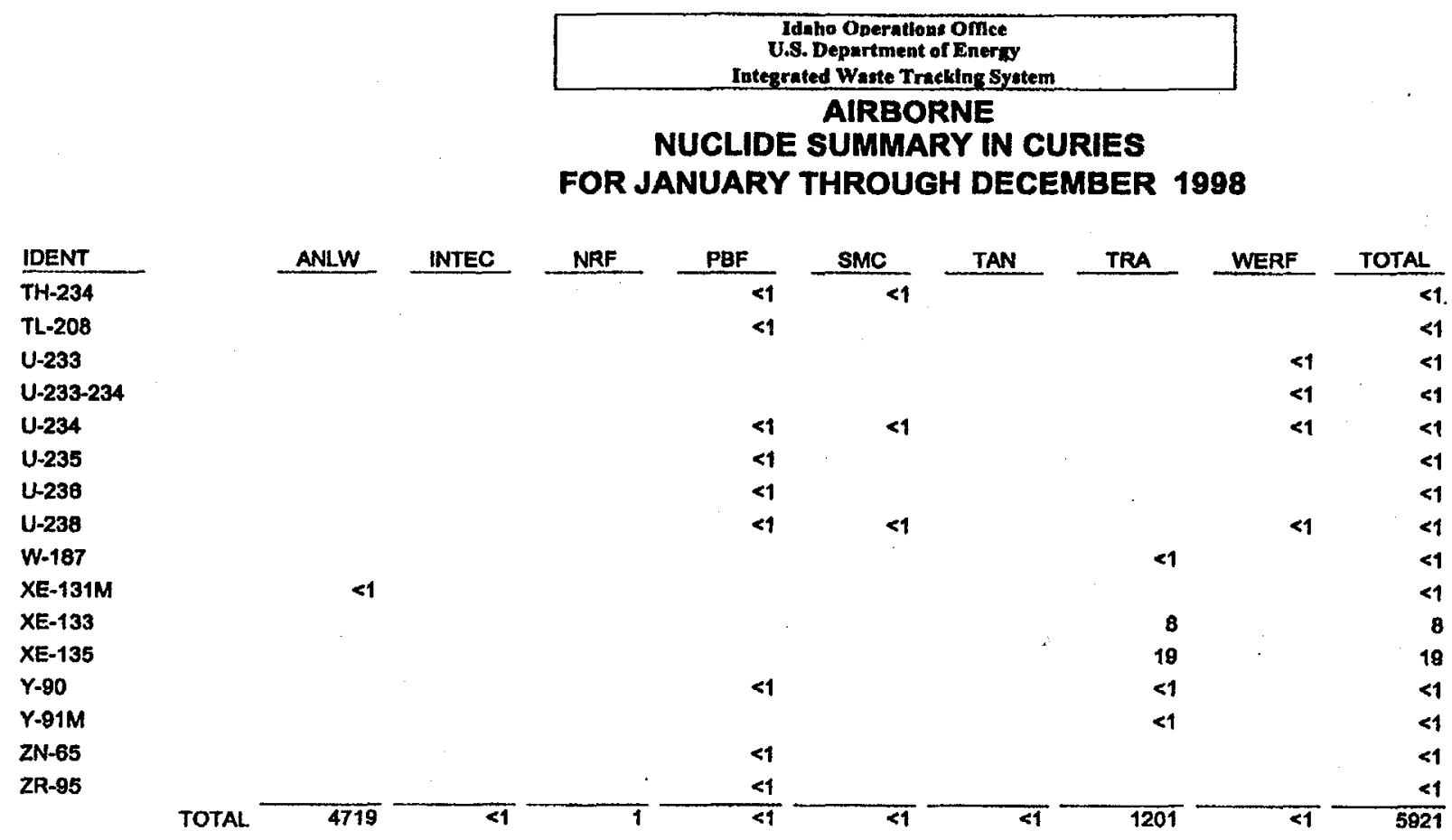




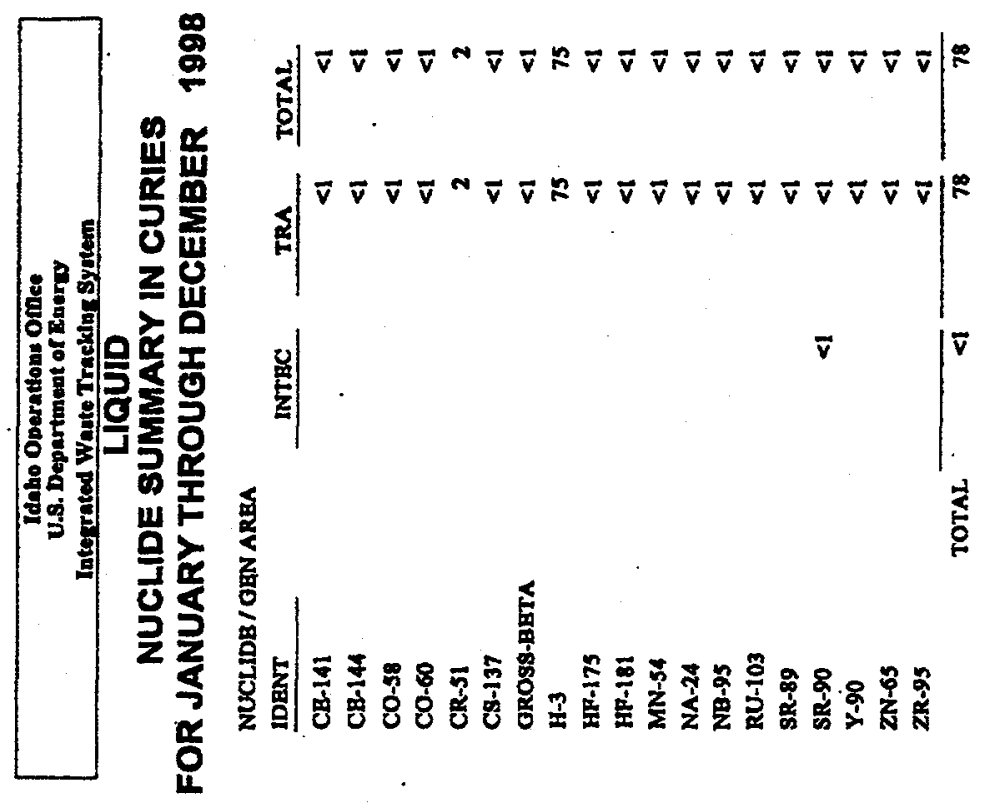




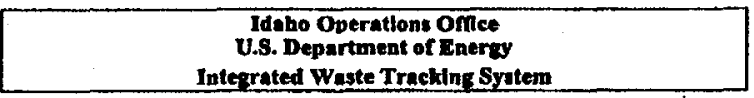

Daste Tracking

DISPOSED SOLID WASTE

FOR JANUARY THROUGH DECEMBER 1998

\begin{tabular}{|c|c|c|c|c|c|c|c|c|c|c|c|c|c|c|}
\hline $\begin{array}{l}\text { NUCLIDE / GBN AREA } \\
\text { IDENT }\end{array}$ & ANLW & $\mathrm{CF}$ & $\mathrm{D}+\mathrm{D}$ & INTEC & NRP & PBF & SMC & TAN & TRA & WAGI & WAO3 & WAO7 & WERF & TOTAL \\
\hline $\mathrm{AC}-225$ & & & & & & & & & & & . & & $<1$ & $<1$ \\
\hline AC-227 & & $<1$ & & & & & & & & & & & $<1$ & $<1$ \\
\hline AC-228 & & & & & & . & & & & & & & $<1$ & $<1$ \\
\hline AO-108 & & & & & & & & & $<1$ & & & & & $<1$ \\
\hline AO-108M & & & & & & & & & $<1$ & & & & $<1$ & $<1$ \\
\hline$A O-110 M$ & $<1$ & & $<1$ & & & $<1$ & & $<1$ & & & & & $<1$ & $<1$ \\
\hline AM-241 & $<1$ & $<1$ & $<1$ & $<1$ & $<1$ & $<1$ & & $<1$ & $<1$ & & & & $<1$ & $<1$ \\
\hline AM-242M & & & & & & & & & & & & & $<1$ & $<1$ \\
\hline AM-243 & & & & & & & & & & & & & $<1$ & $<1$ \\
\hline AT-217 & & & & & & & & & & & & & $<1$ & $<1$ \\
\hline$B A-133$ & & & $<1$ & & & & & & & & & & & $<1$ \\
\hline BA-137 & $<1$ & $<1$ & $<1$ & & & & & & & & & & $<1$ & $<1$ \\
\hline BA-137M & 1 & $<1$ & .4 & $<1$ & $<1$ & & & $<1$ & $<1$ & $<1$ & $<1$ & & $<1$ & 6 \\
\hline BA-140 & & & & & & & & & & & & & $<1$ & $<1$ \\
\hline BI-210 & & & & & & & & & & & & & $<1$ & $<1$ \\
\hline BI-212 & & & & & & & & & . & & & & $<1$ & $<1$ \\
\hline BI-213 & & & & & & & & & & & & & $<1$ & $<1$ \\
\hline BI-214 & & & & & & & & & & & & & $<1$ & $<1$ \\
\hline$C-14$ & & $<1$ & $<1$ & $<1$ & $<1$ & & & & $<1$ & $<1$ & & & $<1$ & $<1$ \\
\hline$C D-113 M$ & $<1$ & & & & & & & & & & & & $<1$ & $<1$ \\
\hline CE-137 & & & & & & & & $<1$ & & & & & $<1$ & $<1$ \\
\hline CE-14I & & & & & & & & & $<1$ & & & & $<1$ & $<1$ \\
\hline CE-144 & 1 & & & & & & & & $<1$ & & & & $<1$ & 2 \\
\hline CM-242 & & $<1$ & & & & & & 4 & & & & & $<1$ & $<1$ \\
\hline CM-244 & & $<1$ & $<1$ & & & & & $<1$ & $<1$ & & & & $<1$ & $<1$ \\
\hline Co.57 & $<1$ & & & & & & & & & & & & $<1$ & $<1$ \\
\hline
\end{tabular}

SD18EN01

REPOS

Run Dato: 05/28/1999

Page 1 of 5 


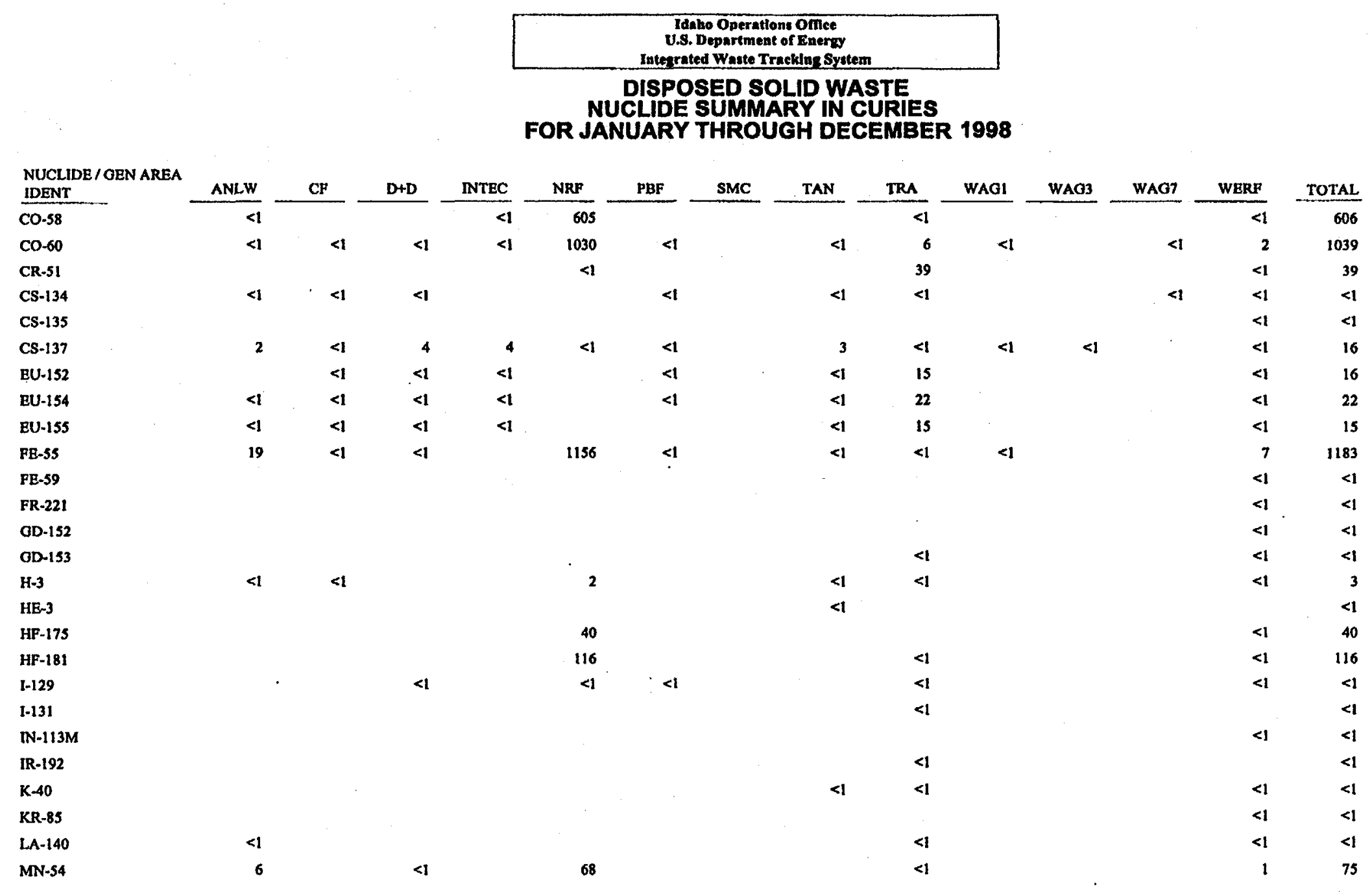




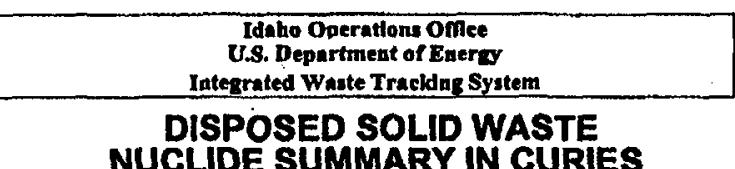

NUCISO SUMMARY WASTES

FOR JANUARY THROUGH DECEMBER 1998

\begin{tabular}{|c|c|c|c|c|c|c|c|c|c|c|c|c|c|c|}
\hline $\begin{array}{l}\text { NUCLIDE / GEN AREA } \\
\text { IDENT }\end{array}$ & ANLW & $\mathrm{CF}$ & $D+D$ & INTEC & NRF & PBF & SMC & TAN & TRA & WAGI & WAG3 & WAG7 & WERF & TOTAL \\
\hline MO-93 & $<1$ & & & & & & & & & & & & $<1$ & $<1$ \\
\hline MO.99 & & & & & & & & & & & & & $<1$ & $<1$ \\
\hline NA-22 & $<1$ & & & & & & & & & & & & $<1$ & $<1$ \\
\hline NB-93M & & & & & $<1$ & & & & & & & & $<1$ & $<1$ \\
\hline NB-94 & & & $<1$ & & $<1$ & & & & & & & & $<1$ & $<1$ \\
\hline NB-95 & $<1$ & & & & 101 & & & & $<1$ & & & & $<1$ & 101 \\
\hline NI-59 & $<1$ & $<1$ & $<1$ & & 16 & & & & $<1$ & $<1$ & & & $<1$ & 16 \\
\hline NI-63 & $<1$ & $<1$ & $<1$ & $<1$ & 1935 & $<1$ & & $<1$ & $<1$ & $<1$ & & & $<1$ & 1937 \\
\hline NP-237 & & & $<1$ & & & & & & & & & & $<1$ & $<1$ \\
\hline NP.239 & & & & & & & & & & & & & $<1$ & $<1$ \\
\hline PA-231 & & $<1$ & & & & & & & & & & & $<1$ & $<1$ \\
\hline PA-234 & & $<1$ & & & & & & & & & & & $<1$ & $<1$ \\
\hline PA-234M & & $<1$ & & & & & $<1$ & & $<1$ & & & & $<1$ & $<1$ \\
\hline PB-209 & & & & & . & & & & & & & & $<1$ & $<1$ \\
\hline PB-210 & & & & & & & & & & & & & $<1$ & $<1$ \\
\hline PB.212 & & & & & & & & & & & & & $<1$ & $<1$ \\
\hline PB.214 & & & & & & & & & & & & & $<t$ & $<1$ \\
\hline PD.107 & & & & & & & & & & & & & $<1$ & $<1$ \\
\hline PM-147 & 3 & $<1$ & 20 & $<1$ & & & & $<1$ & $<1$ & $<1$ & $<1$ & & $<1$ & 24 \\
\hline PO-210 & & & & & & & & & $<1$ & & & & $<1$ & $<1$ \\
\hline PO-212 & & & & & & & & & & & & & $<1$ & $<1$ \\
\hline PO.213 & & & & & & & & & & & & & $<1$ & $<1$ \\
\hline$P 0.214$ & & & & & & & & & & & & & $<1$ & $<1$ \\
\hline PO-216 & & & & & & & & & & & & & $<1$ & $<1$ \\
\hline PO-218 & & & & & & & & & & & & & $<1$ & $<1$ \\
\hline PR-144 & 1 & & & & & & & & & & & & $<1$ & 2 \\
\hline
\end{tabular}




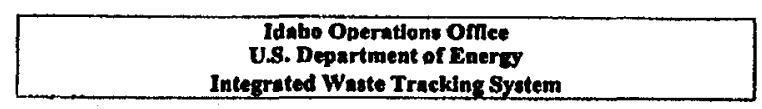

Integrated Waste Tracking System

UCLIDE SUMMAR IASTE

FOR JANUARY THROUGH DECEMBER 1998

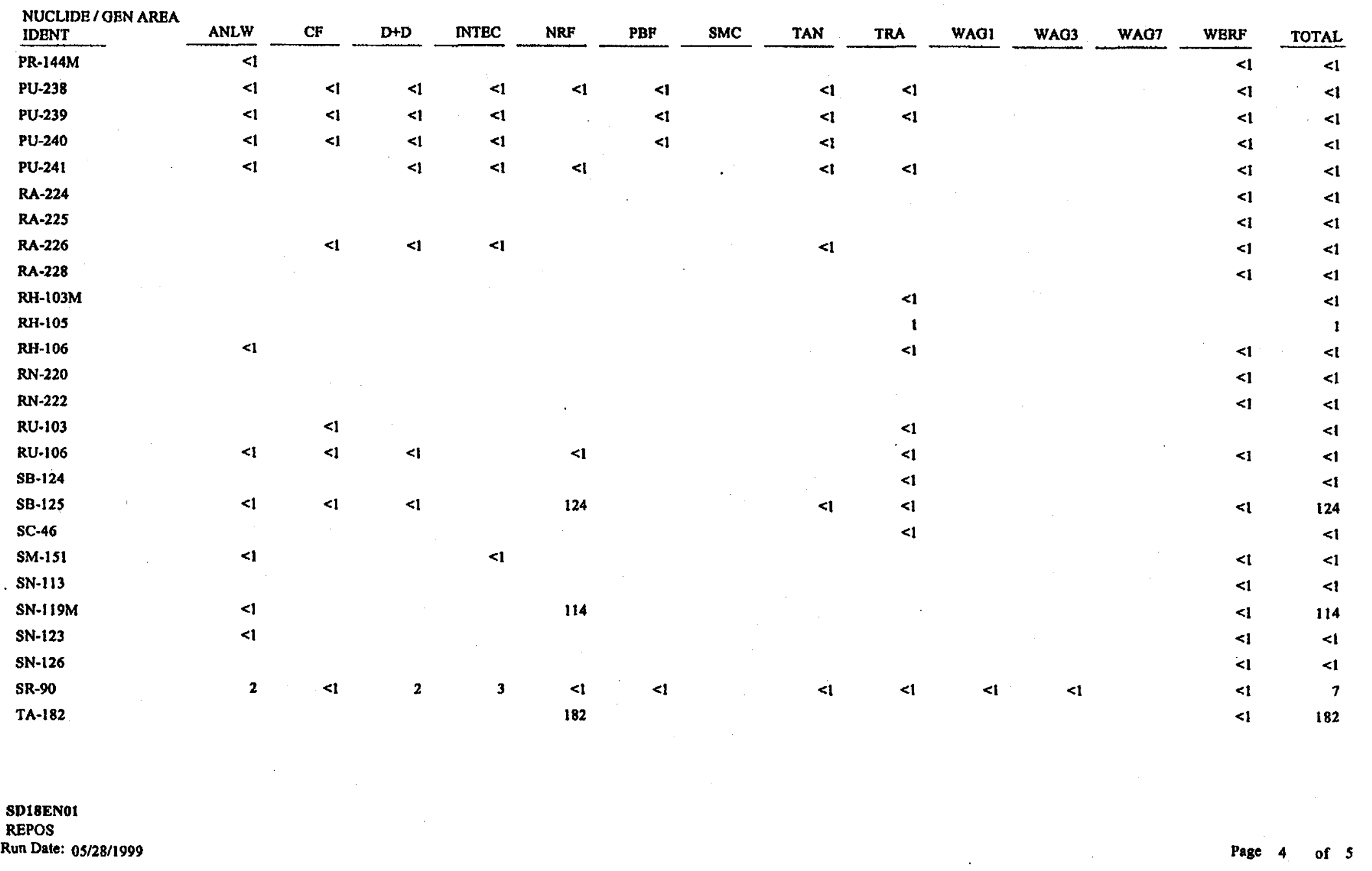




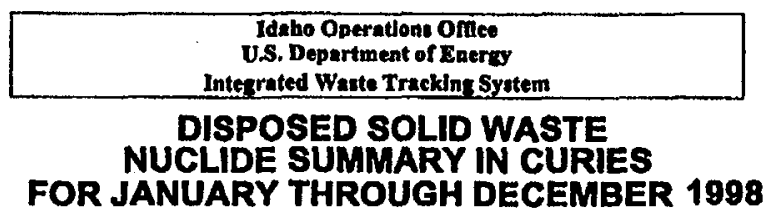

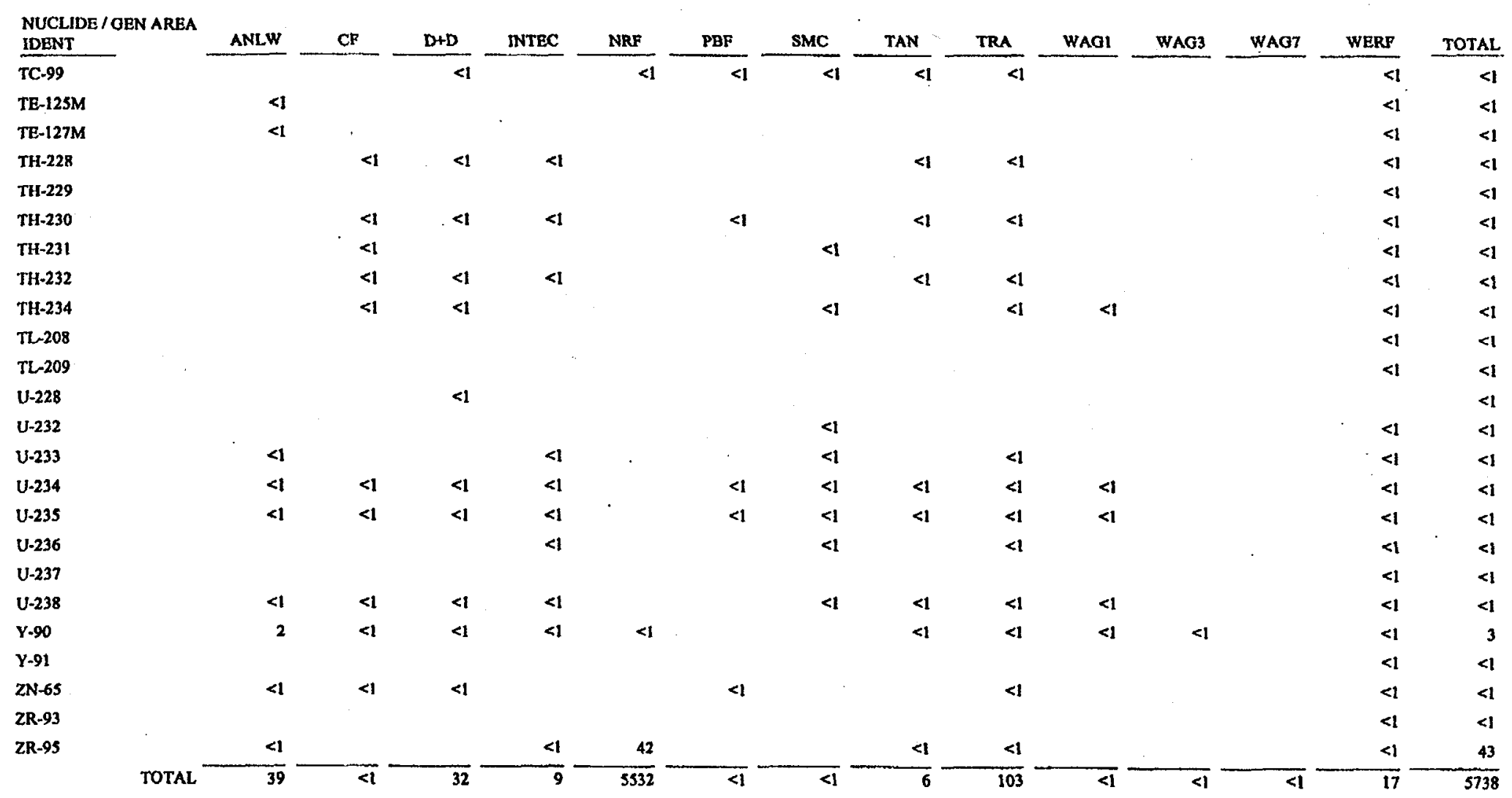




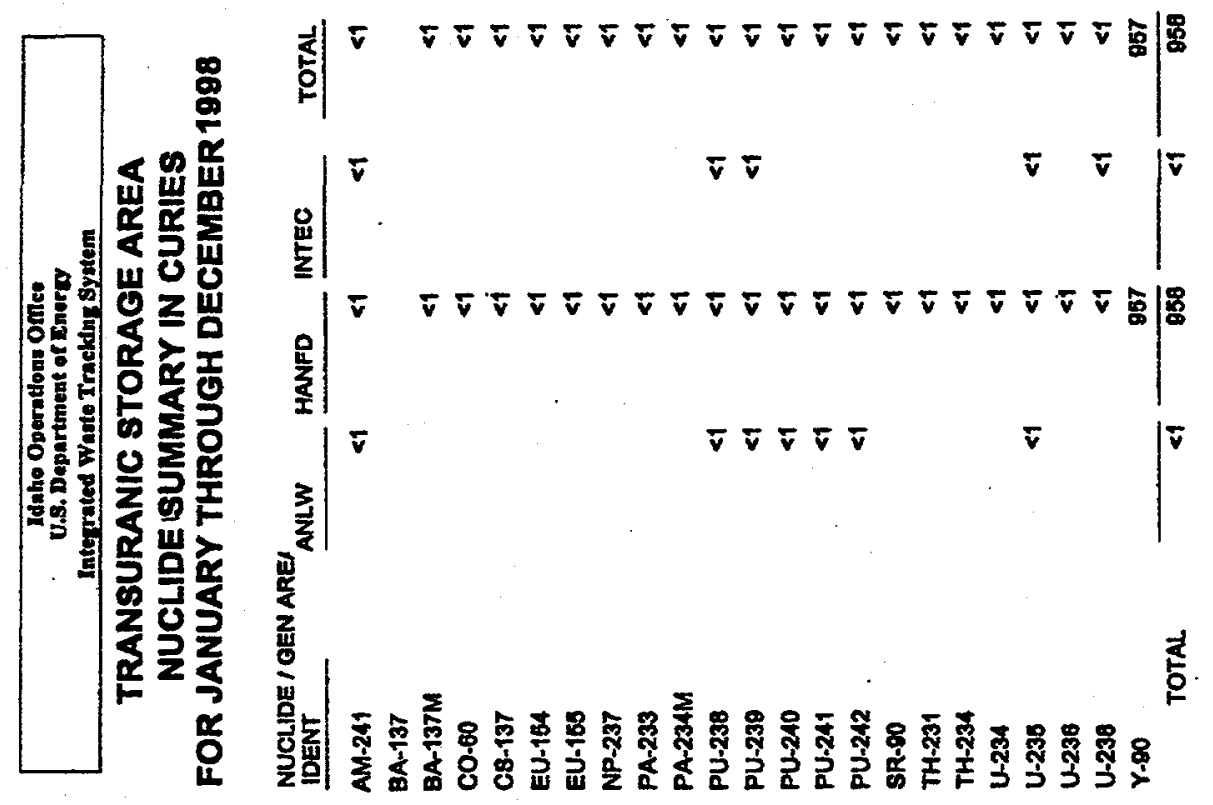

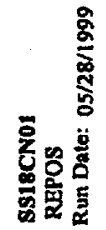




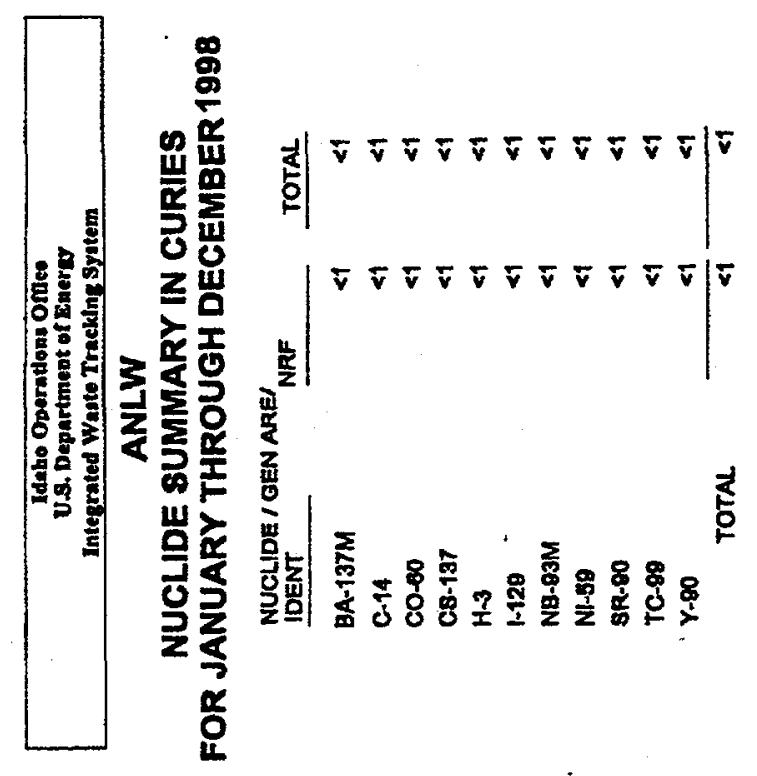




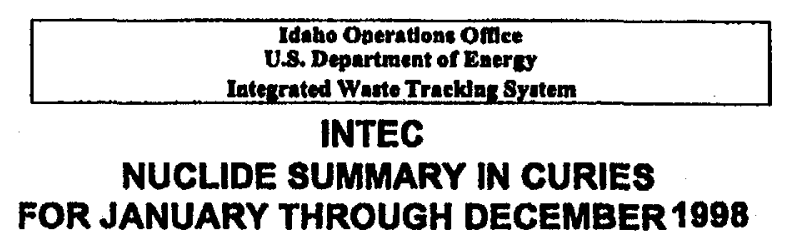

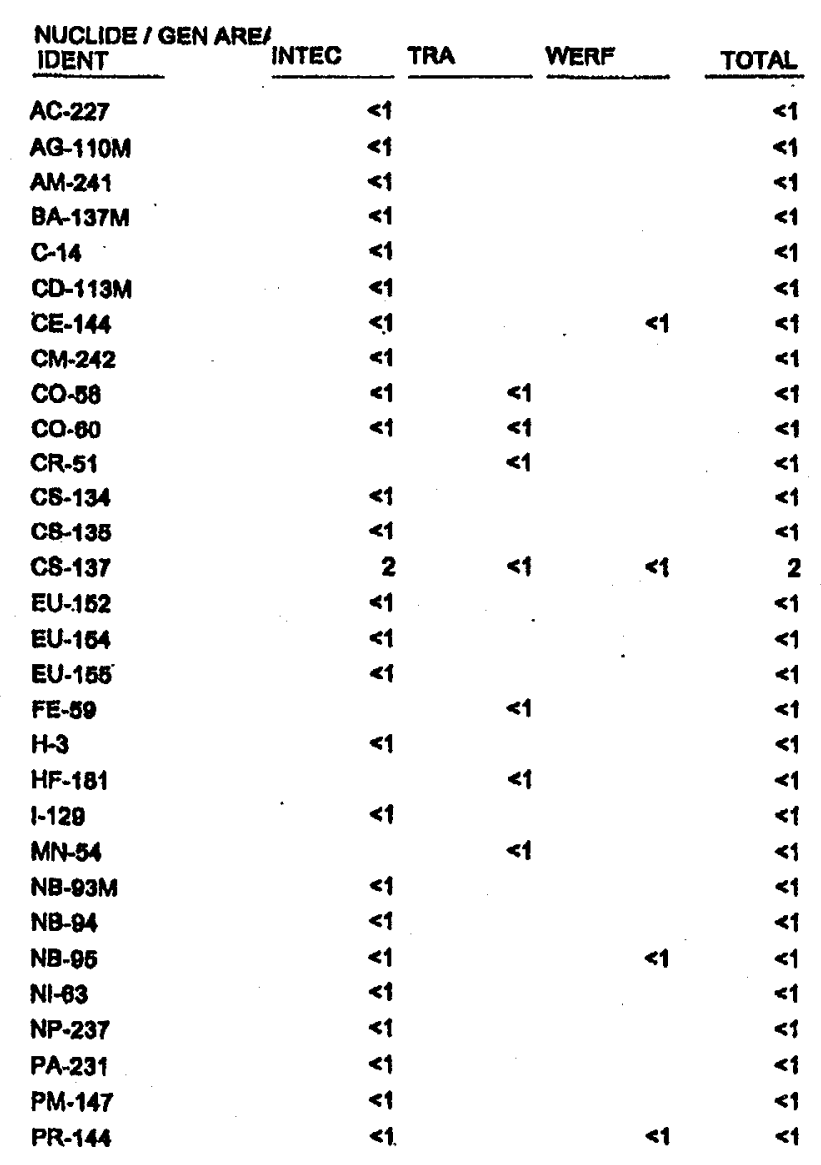




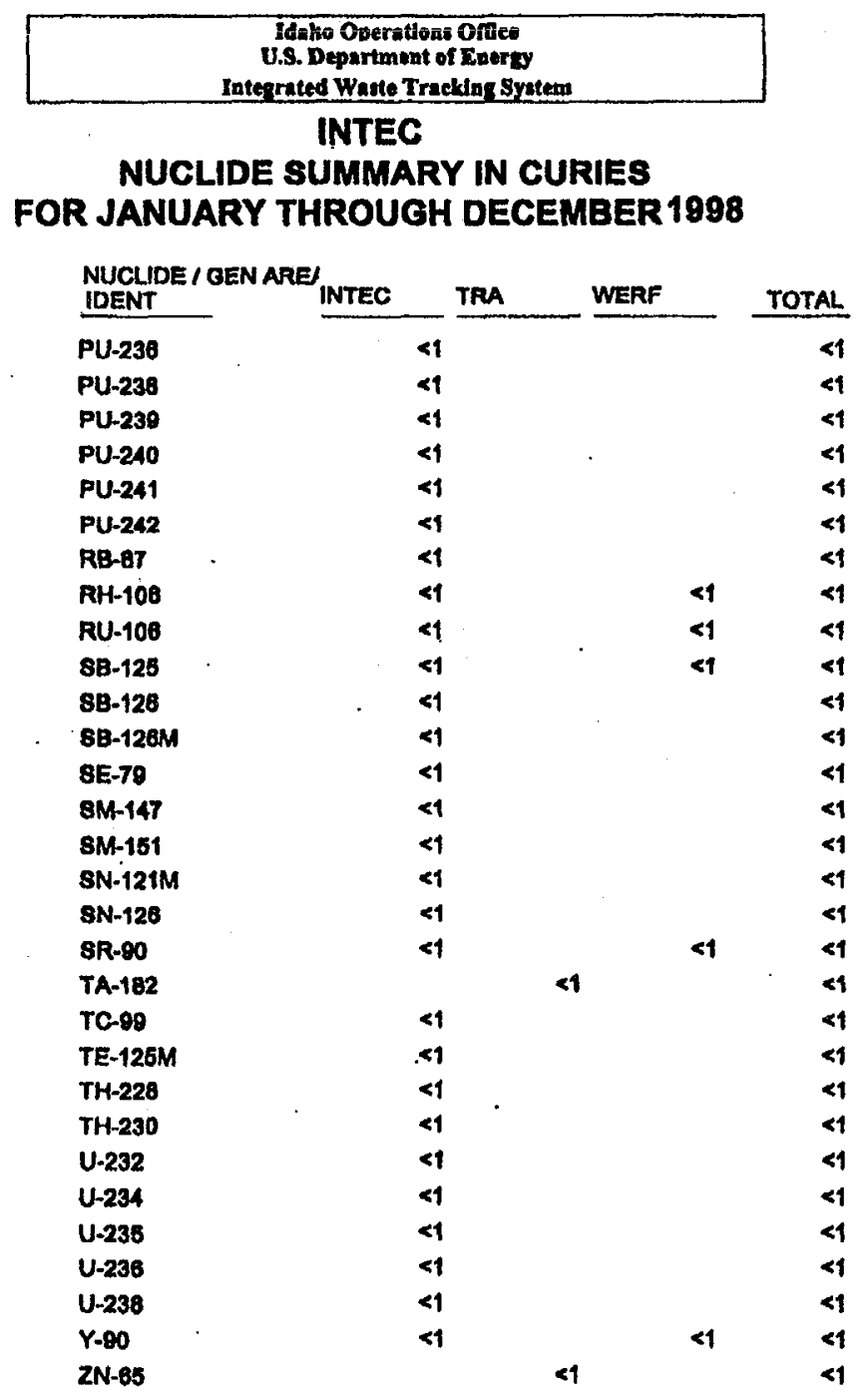




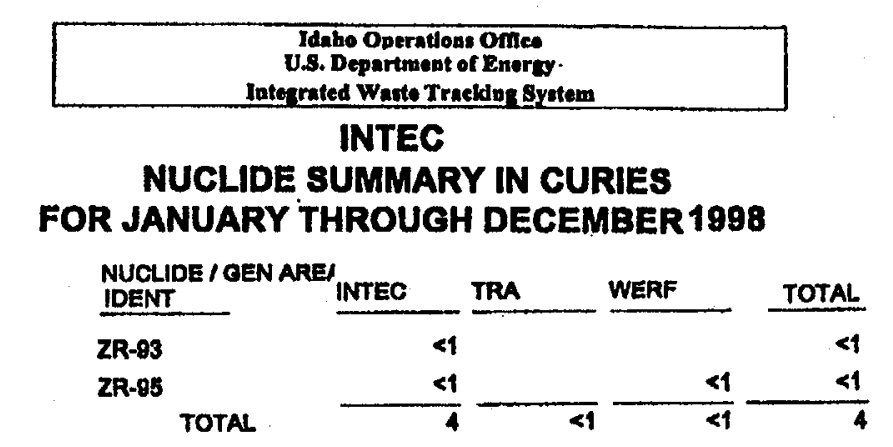

具 


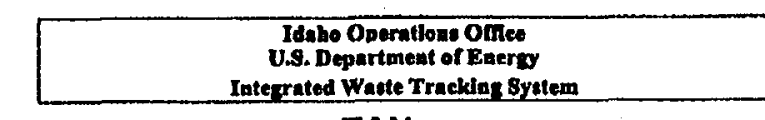

TAN

NUCLIDE SUMMARY IN CURIES

FOR JANUARY THROUGH DECEMBER 1998

\begin{tabular}{|c|c|c|}
\hline $\begin{array}{l}\text { NUCLIDE / GEN ARE } \\
\text { IDENT }\end{array}$ & TAN & TOTAL \\
\hline AM-241 & $<1$ & $<1$ \\
\hline BA-137 & $<1$ & $<1$ \\
\hline$C 0-60$ & $<1$ & $<1$ \\
\hline C8-134 & $<1$ & $<1$ \\
\hline C8-137 & $<1$ & $<1$ \\
\hline EU-164 & $<1$ & $<1$ \\
\hline FE-56 & $<1$ & $<1$ \\
\hline $1-129$ & $<1$ & $<1$ \\
\hline NI-63 & $<1$ & $<1$ \\
\hline RH-100 & $<1$ & $<1$ \\
\hline RU-100 & $<1$ & $<1$ \\
\hline $88-125$ & $<1$ & $<t$ \\
\hline 8R-90 & $<1$ & $<1$ \\
\hline TC-89 & $<1$ & $<1$ \\
\hline Y.80 & $<1$ & $<1$ \\
\hline TOTAL & 4 & $<$ \\
\hline
\end{tabular}




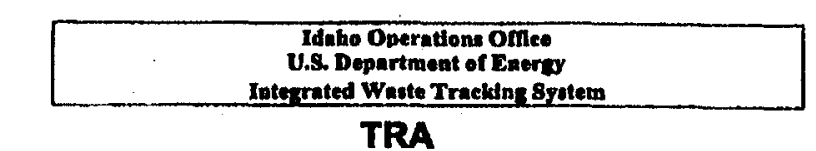

NUCLIDE SUMMARY IN CURIES

FOR JANUARY THROUGH DECEMBER 1998

$\frac{\text { NUCLIDE I GEN AREI WERF }}{\text { CS-137 }}$
$\frac{41}{\text { TOTAL. }}$

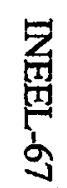




\begin{tabular}{|c|c|c|c|c|c|c|c|c|c|c|c|c|c|}
\hline \multirow[b]{3}{*}{$\begin{array}{l}\text { NUCLIDE / GEN ARE/ } \\
\text { IDENT }\end{array}$} & \multicolumn{8}{|c|}{ 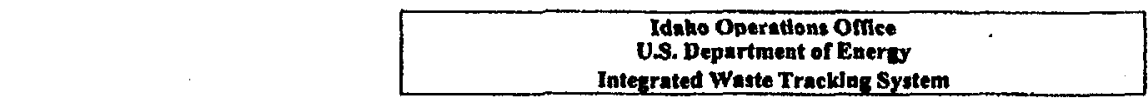 } & \multirow[b]{3}{*}{ WERF } & \multirow[b]{3}{*}{ WMF } & \multirow{3}{*}{\multicolumn{3}{|c|}{ TOTAL }} \\
\hline & \multirow{2}{*}{$-\mathrm{CF}$} & \multirow[b]{2}{*}{$D+D$} & \multirow[b]{2}{*}{ IsU } & \multirow[t]{2}{*}{ OR J/ } & $\begin{array}{l}\text { CLIDE } \\
\text { UARY }\end{array}$ & $\begin{array}{l}\text { ERF } \\
\text { AMARY IN } \\
\text { OUGH DE }\end{array}$ & $\begin{array}{l}\text { CURIE } \\
\text { ECEMB }\end{array}$ & $\begin{array}{l}\text { S } \\
\text { SR1998 }\end{array}$ & & & & & \\
\hline & & & & & SMC & TAN & TRA & WAGT & & & & & \\
\hline AC-227 & & & & & & & $<1$ & & & 1 & & & \\
\hline AC-228 & & $<1$ & & & & & & & & 1 & & & $<1$ \\
\hline AG-108 & & $<1$ & & & & & & & & & & & \\
\hline AG-108M & & & & & & & & & & 1 & & & $<1$ \\
\hline AG-110 & & $<1$ & & & & & & & & 1 & & & $<1$ \\
\hline AG-110M & $<1$ & & & $\cdot$ & & & & & & 1 & & & \\
\hline AM-241 & $<1$ & $<1$ & & & $<1$ & $<1$ & $<1$ & & & 1 & $<1$ & & $<1$ \\
\hline AM-243 & & & & & & & & & & 1 & & & $<1$ \\
\hline BA-133 & & $<1$ & & & & & & & & & & & $<1$ \\
\hline BA-137 & & & & & & & & & & e1 & & & $<1$ \\
\hline BA-137M & $<1$ & & & $<1$ & $<1$ & $<1$ & $<1$ & & & $=1$ & & & $<1$ \\
\hline B1-212 & & $<4$ & & & & $<4$ & & & & $<1$ & & & \\
\hline B1-214 & & $<1$ & & & & & & & & & & & $<1$ \\
\hline C-14 & & $<1$ & & & $<1$ & & $<1$ & & & 81 & & & $<1$ \\
\hline CD-109 & & $<1$ & & & & & & & & & & & $<1$ \\
\hline CD-113 & & & & & & & & & & e1 & & & $<1$ \\
\hline CD-113M & $<1$ & & & & & & & & & $<1$ & & & $<1$ \\
\hline CE-134 & & & & & & $<1$ & & & & & & & $<1$ \\
\hline CE-137 & & & & & & $<1$ & & & & & & & $<1$ \\
\hline CE-139 & & $<1$ & & & & & & & & & & & $<1$ \\
\hline CE-14t & & & & & & & & & & $: 1$ & & & $<1$ \\
\hline CE-144 & $<1$ & $<1$ & & & $<1$ & & & & & 61 & & & $<1$ \\
\hline CM-244 & $<1$ & $<1$ & & & $<1$ & & & & & $=1$ & & & $<1$ \\
\hline CO-57 & & $<1$ & & & $<1$ & & & & & $<1$ & & & $<1$ \\
\hline CO-58 & $<1$ & $<1$ & & & $<1$ & & $<1$ & & & $<1$ & & & $<1$ \\
\hline$C 0-60$ & $<1$ & $<1$ & $<1$ & & $<1$ & $<1$ & $<1$ & $<1$ & & $<1$ & & $<1$ & $<1$ \\
\hline CR-51 & & & & & $<1$ & & $<1$ & & & <9 & & & $<1$ \\
\hline CS-134 & $<1$ & $<1$ & & & $<1$ & $<1$ & $<1$ & $<1$ & & <1 & & $<1$ & $<1$ \\
\hline CS-135 & $<1$ & & & & & & & & & & & & $<1$ \\
\hline CS-137 & $<1$ & $<1$ & $<1$ & $<1$ & $<1$ & $<1$ & $<1$ & & & $<1$ & & $\ll 1$ & $<1$ \\
\hline
\end{tabular}


章
量
s

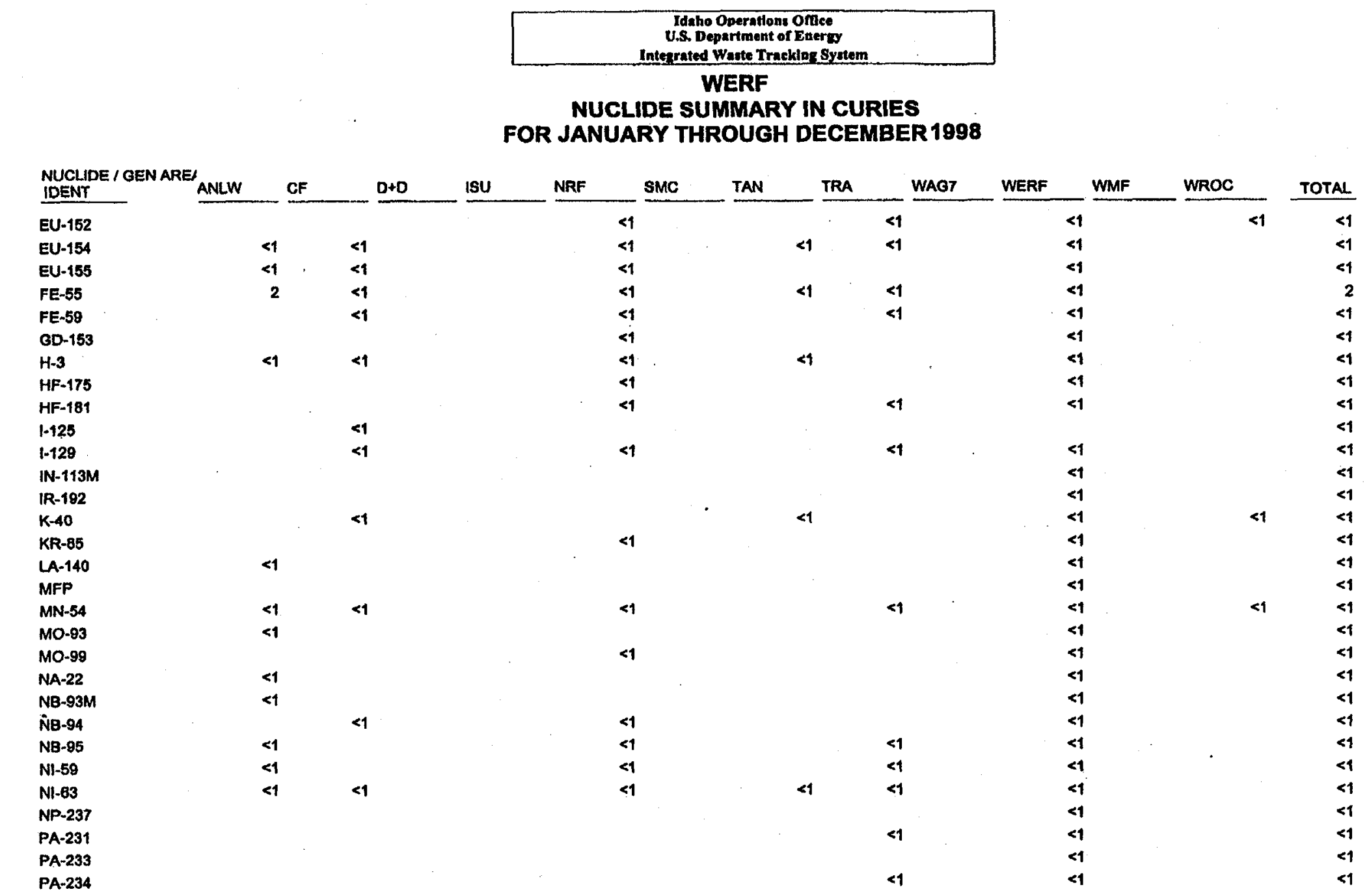

\section{SS18CN01}

REPOS

Run Date: 05/28/1999 


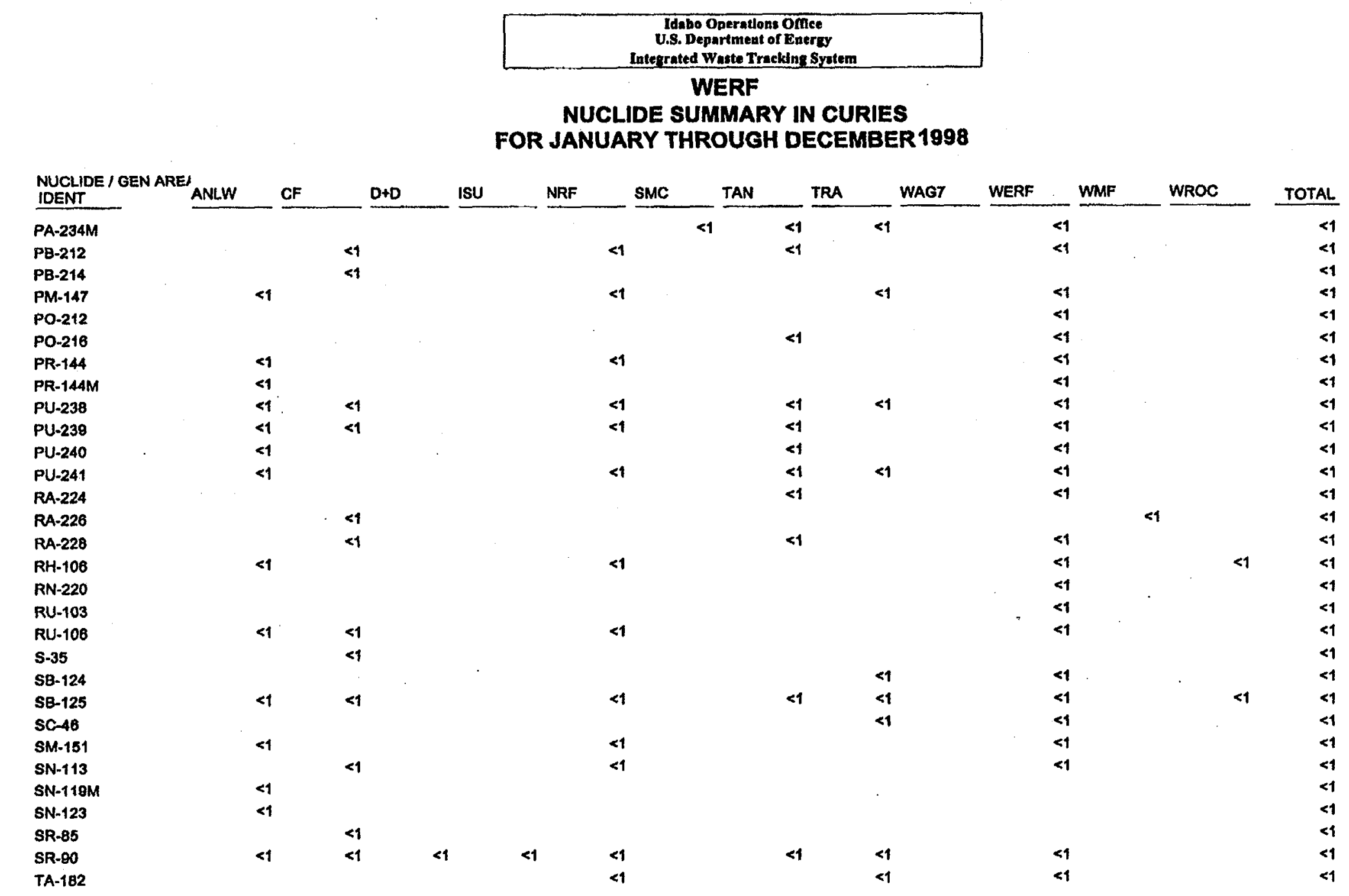




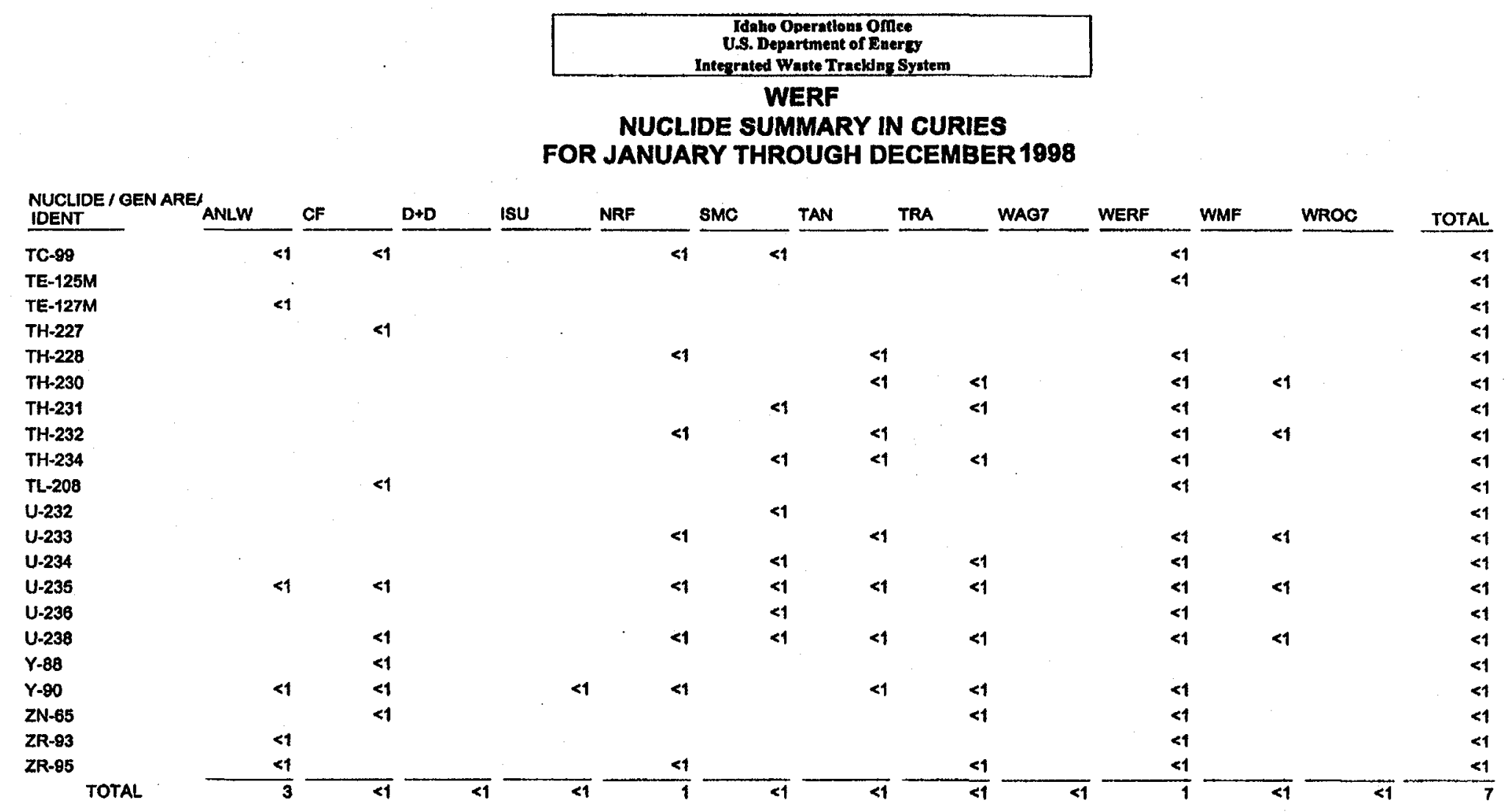




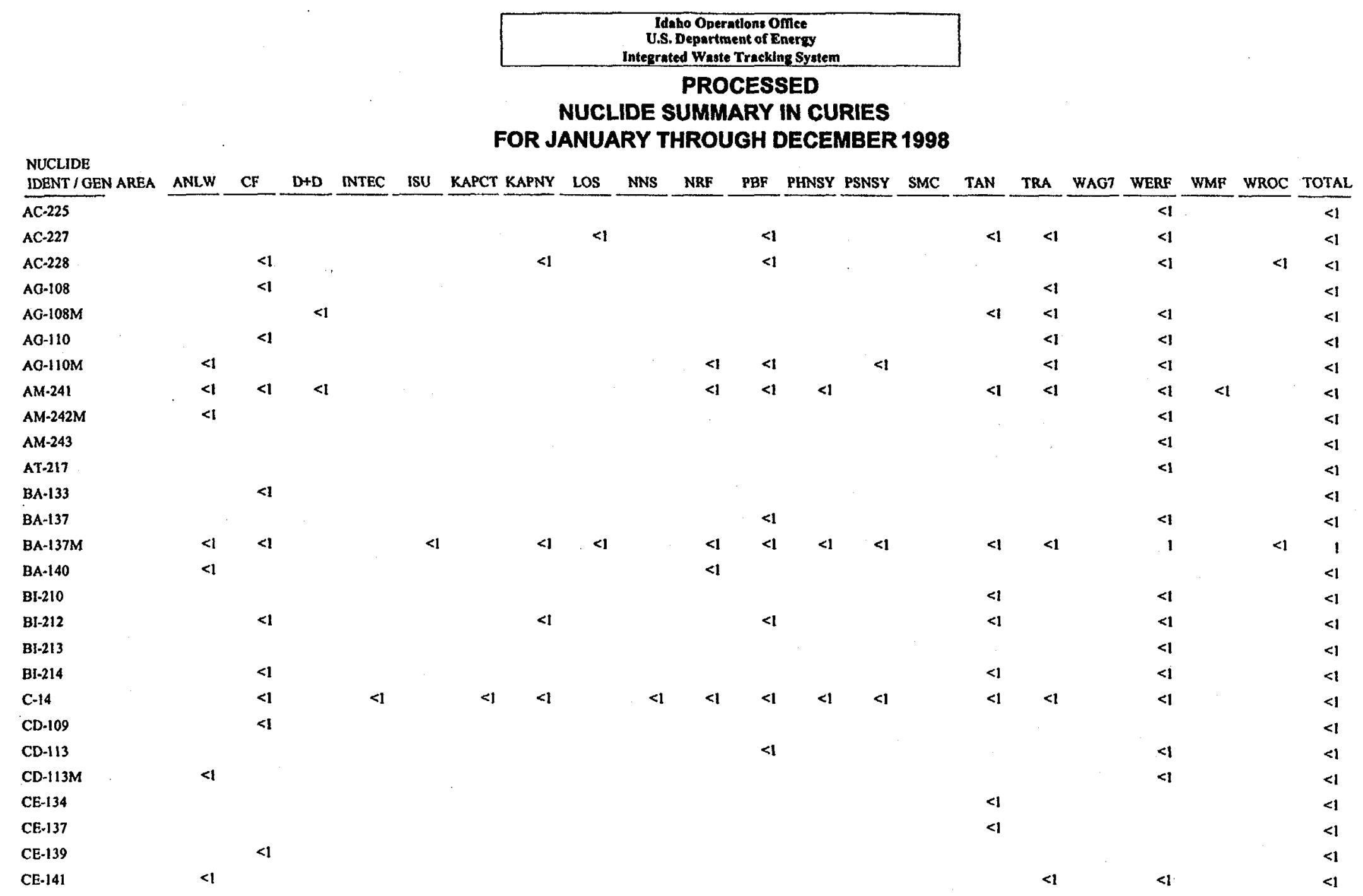

\section{SP18GNO1}

Run Date: 06/03/1999 


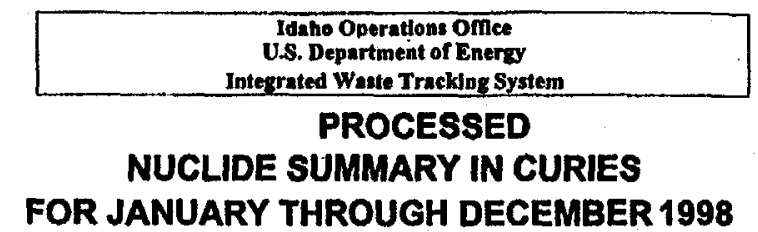

NUCLIDE

FOR JANUARY THROUGH DECEMBER 1998

\begin{tabular}{|c|c|c|c|c|c|c|c|c|c|c|c|c|c|c|c|c|c|c|c|c|c|}
\hline IDENT / GEN AREA & ANLW & $\mathrm{CF}$ & $D+D$ & INTEC & IsU & KAPCT & KAPNY & Los & NNS & $\mathrm{NRF}$ & $\mathrm{PBF}$ & PHNSY & PSNSY & SMC & TAN & TRA & WAG7 & WERF & WMF & WROC & TOTAL \\
\hline CE-144 & $<1$ & $<1$ & & $<1$ & & & & & & $<1$ & $<1$ & & $<1$ & & & $<1$ & & $<1$ & & & $<1$ \\
\hline CF-249 & & & & & & & & & & & & & & & & & & $<1$ & & & $<1$ \\
\hline $\mathrm{CF}-251$ & & & & & & & & & & & & & & & & & & $<1$ & & & $<1$ \\
\hline CM-242 & & & & & & & & & & & $<1$ & & & & $<1$ & & & $<1$ & & & $<1$ \\
\hline CM-243 & & & & & & & & & & & $<1$ & & & & & & & $<1$ & & & $<1$ \\
\hline CM-244 & $<1$ & $<1$ & $<1$ & & & & & & & $<1$ & $<1$ & $<1$ & & & $<1$ & & & $<1$ & & & $<1$ \\
\hline CM-245 & & & & & & & & & & & $<1$ & & & & & & & $<1$ & & & $<1$ \\
\hline CM-246 & & & & & & & & & & & $<1$ & & & & & & & $<1$ & & & $<1$ \\
\hline CM-247 & & & & & & & & & & & & & & & & & & $<1$ & & & $<1$ \\
\hline CM-248 & & & & & & & & & & & & & & & & & & $<1$ & & & $<1$ \\
\hline Co. 57 & & $<1$ & & $<1$ & & & & & & $<1$ & $<1$ & & $<1$ & & & $\because$ & & $<1$ & & $<1$ & $<1$ \\
\hline$C 0-58$ & $<1$ & $<1$ & & $<1$ & & & $<$ & & $<1$ & $<$ & $<1$ & & $<1$ & & & $<1$ & & $<1$ & & & $<1$ \\
\hline Co.60 & $<1$ & $<1$ & $<1$ & $<1$ & & $<1$ & $<1$ & & $<1$ & 2 & $<1$ & $<1$ & $<1$ & & $<1$ & $<1$ & $<1$ & $<1$ & & $<1$ & 4 \\
\hline CR-SI & & & & & & & & . & & $<$ & & & & & & $<1$ & & $<1$ & & & $<1$ \\
\hline CS-134 & $<1$ & $<1$ & & & & & & & & $<1$ & $<1$ & & $<1$ & & $<1$ & $<1$ & $<1$ & $<1$ & & $<1$ & $<1$ \\
\hline CS-135 & $<1$ & & & & & & & & & & & & & & & & & $<1$ & & & $<1$ \\
\hline CS-137 & $<1$ & $<1$ & $<1$ & $<1$ & $<1$ & & $<1$ & $<1$ & & $<1$ & $<1$ & $<1$ & $<1$ & & $<1$ & $<1$ & & 1 & & $<1$ & 2 \\
\hline EU-152 & $<1$ & & & $<1$ & & $<1$ & & & & $<1$ & $<1$ & $<1$ & & & & $<1$ & & $<1$ & & $<1$ & $<1$ \\
\hline EU-154 & $<1$ & $<1$ & & $<1$ & & $<1$ & & & & $<1$ & $<1$ & $<1$ & $<1$ & & $<1$ & $<1$ & & $<1$ & & & $<1$ \\
\hline EU-15s & $<1$ & $<1$ & & & & & & & & $<1$ & $<1$ & $<1$ & $<1$ & & & $<1$ & & $<1$ & & & $<1$ \\
\hline FE-5S & 4 & $<1$ & & $<1$ & & $<1$ & $<1$ & & $<1$ & 3 & $<1$ & $<1$ & $<1$ & & $<1$ & $<1$ & & 10 & & & 17 \\
\hline FE-59 & & $<1$ & & & & & & & & $<1$ & $<1$ & & $<1$ & & & $<1$ & & $<1$ & & & $<1$ \\
\hline FR-221 & & & & & . & & & & & & & & & & & & & $<1$ & & & $<1$ \\
\hline GD-152 & & & & & & & & & & & & & & & & & & $<1$ & & & $<1$ \\
\hline GD-153 & & & & & & & & & & $<1$ & & & & & & & & $<1$ & & & $<1$ \\
\hline $\mathrm{H}-3$ & $<1$ & $<1$ & & & & $<1$ & $<1$ & $<1$ & & $<1$ & $<1$ & & $<1$ & & $<1$ & & & $<1$ & & & $<1$ \\
\hline $\mathrm{HF} \cdot 175$ & & & & & & & & & & $<1$ & $<1$ & & $<1$ & & & & & $<1$ & & & $<1$ \\
\hline
\end{tabular}




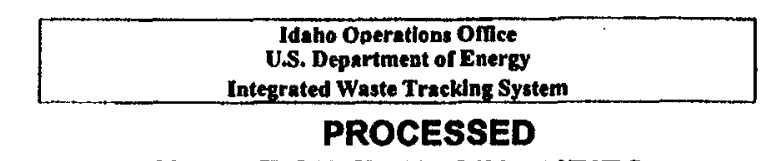

NUCLIDE SUMMARY IN CURIES

FOR JANUARY THROUGH DECEMBER 1998

NUCLIDE

IDENT/GEN AREA ANLW CF D+D INTEC ISU KAPCT KAPNY LOS NNS NRF PBF PHNSY PSNSY SMC TAN TRA WAG7 WERF WMF WROC TOTAL

HF-181

I-125

I 129

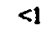

I.131

IN-II3M

IR-192

$\mathrm{K}-40$

KR-85

LA-140

MFP

MN-53

MN-54

MO-93

MO-99

NA-22

NB-93M

NB-94

NB.95

NI-59

NI-63

NP-237

NP-239

P-32

PA-231

PA-233

PA-234

PA-234M

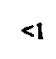

$<1$

$<1$

$<1$

$<1$

$<1$

$<<<$

$<1<1<1$

$<1$

\begin{tabular}{|c|c|c|c|c|c|c|c|}
\hline \multirow[t]{3}{*}{$<1$} & $<1$ & $<1$ & $<1$ & $<1$ & $<1$ & $<1$ & \\
\hline & & & & & $<1$ & & \\
\hline & & & & $<1$ & $<1$ & $<1$ & $<1$ \\
\hline \multirow[t]{3}{*}{$<1$} & & & & & $\Leftrightarrow 1$ & $<1$ & $<1$ \\
\hline & $<1$ & & & & $<1$ & $<1$ & \\
\hline & & $<1$ & & & $<1$ & $<1$ & $<1$ \\
\hline$<1$ & $<1$ & $<1$ & $<1$ & $<1$ & $<1$ & $<1$ & $<1$ \\
\hline
\end{tabular}

$<$

$<1$

$<$

SP18GN01

REPOS

Run Date: 06/03/1999 


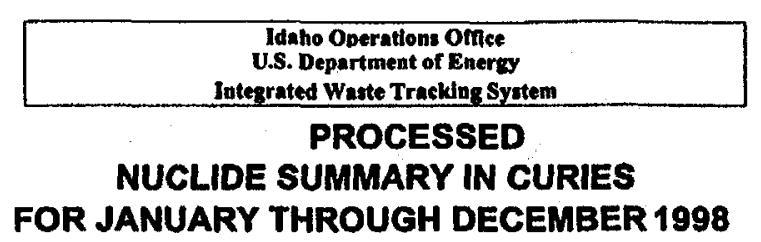

NUCLIDE FOR JANUARY THROUGH DECEMBER 1998

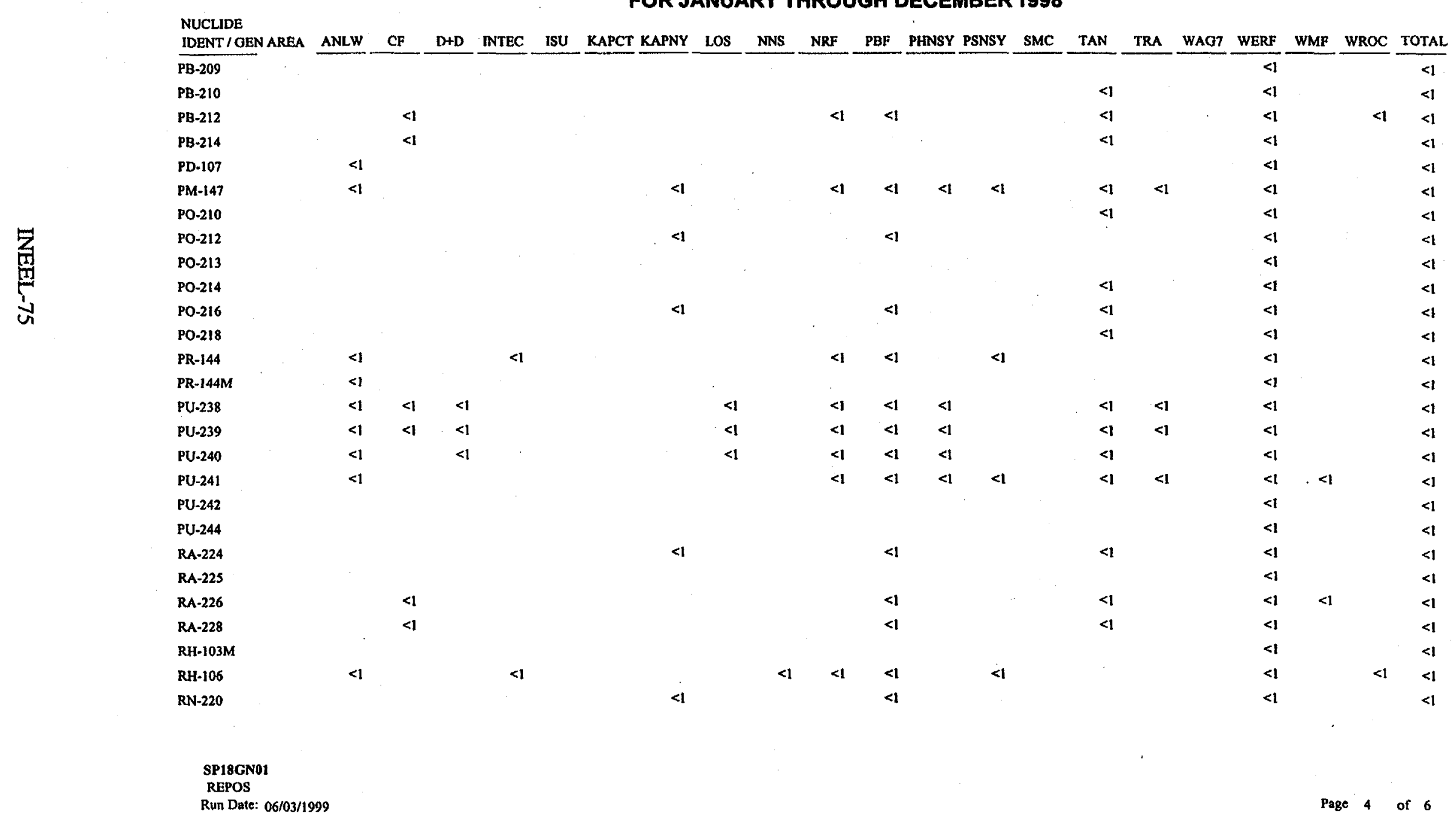




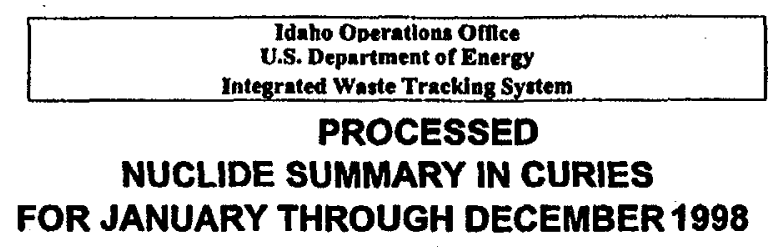

\begin{tabular}{|c|c|c|c|c|c|c|c|c|c|c|c|c|c|c|c|c|c|c|c|c|c|}
\hline $\begin{array}{l}\text { NUCLIDE } \\
\text { IDENT / GEN AREA }\end{array}$ & ANLW & $\mathrm{CF}$ & $\mathrm{D}+\mathrm{D}$ & INTEC & ISU & KAPCT & KAPNY & Los & NNS & NRF & PBF & PHNSY & PSNSY & SMC & TAN & TRA & WAG7 & WERF & WMF & WROC & TOTAL, \\
\hline $\mathrm{RN} \cdot 222$ & & & & & & & & & & & & & & & $<1$ & & & $<1$ & & & $<1$ \\
\hline RU-103 & & & & $<1$ & & & & & & & & & & & & $<1$ & & $<1$ & & & $<1$ \\
\hline RU-106 & $<1$ & $<1$ & & $<1$ & & & & & $<1$ & $<1$ & $<1$ & & $<1$ & & & & & $<1$ & & & $<1$ \\
\hline S-35 & & $<1$ & & & & & & & & & & & & & & & & $<1$ & & & $<1$ \\
\hline SB-124 & & & & & & & & & & $<1$ & $<1$ & & $<1$ & & & $<1$ & & $<1$ & & & $<1$ \\
\hline SB-125 & $<1$ & $<1$ & & $<1$ & & $<1$ & $<1$ & & $<1$ & $<1$ & $<1$ & $<1$ & $<1$ & & $<1$ & $<1$ & & $<1$ & & $<1$ & $<1$ \\
\hline$S C-46$ & & & & & & & & & & & & & & & & $<1$ & & $<1$ & & & $<1$ \\
\hline SE-79 & & & & & & & & & & & & & & & & & & $<1$ & & & $<1$ \\
\hline$S M-151$ & $<1$ & & & & & & & & & $<1$ & $<1$ & $<1$ & & & & & & $<1$ & & & $<1$ \\
\hline$S N-113$ & & $<1$ & & & & & & & & $<1$ & $<1$ & & $<1$ & & & & & $<1$ & & & $<1$ \\
\hline SN-119M & $<1$ & & & & & & & & & & & & & & & & & $<1$ & & & $<1$ \\
\hline $\mathrm{SN}-123$ & $<1$ & & & & & & & & & & & & & & & & & $<1$ & & & $<1$ \\
\hline $\mathrm{SN}-126$ & $<1$ & & & & & & & & & & & & & & & & & $<1$ & & & $<1$ \\
\hline SR-85 & & $<1$ & & & & & & & & & & & & & & & & & & & $<1$ \\
\hline SR-90 & $<1$ & $<1$ & $<1$ & $<1$ & $<1$ & & $<1$ & $<1$ & & $<1$ & $<1$ & $<1$ & $<1$ & & $<1$ & $<1$ & & $<1$ & & & $<1$ \\
\hline TC-99 & $<1$ & $<1$ & & $<1$ & & & & $<1$ & & $<1$ & $<1$ & $<1$ & $<1$ & $<1$ & & & & $<1$ & & & $<1$ \\
\hline TE-125M & & & & & & & & & & $<1$ & $<1$ & & & & & & & $<1$ & & & $<1$ \\
\hline TE-127M & $<1$ & & & & & & & & & & & & & & & & & $<1$ & & & $<1$ \\
\hline TH-227 & & $<1$ & & & & & & & & & & & & & & & & & & & $<1$ \\
\hline TH-228 & & & $<1$ & & & & $<1$ & & & $<1$ & $<1$ & & & & $<1$ & & & $<1$ & & $<1$ & $<1$ \\
\hline TH-229 & & & & & & & & & & & & & & & & & & $<1$ & & & $<1$ \\
\hline TH-230 & & & $<1$ & & & & $<1$ & & & & $<1$ & & & & $<1$ & $<1$ & & $<1$ & $<1$ & $<1$ & $<1$ \\
\hline TH-231 & & & & & & & & $<1$ & & $<1$ & $<1$ & & & $<1$ & $<1$ & $<1$ & & $<1$ & & & $<1$ \\
\hline TH-232 & & & $<1$ & & & & $<1$ & & & $<1$ & $<1$ & & & & $<1$ & & & $<1$ & $<1$ & $<1$ & $<1$ \\
\hline TH-294 & & $<1$ & & & & & $<1$ & $<1$ & & $<1$ & $<1$ & & & $<1$ & $<1$ & $<1$ & & $<1$ & & & $<1$ \\
\hline
\end{tabular}




\section{\begin{tabular}{|c|}
\hline $\begin{array}{c}\text { Idaho Operations Omice } \\
\text { U.S. Department of Energy } \\
\text { Integrated Waste Tracklug System }\end{array}$ \\
\hline
\end{tabular} \\ PROCESSED \\ NUCLIDE SUMMARY IN CURIES \\ FOR JANUARY THROUGH DECEMBER 1998}

NUCLIDE

IDENT/GEN AREA ANLW CF D+D NNTEC ISU KAPCT KAPNY LOS NNS NRF PBF PHNSY PSNSY SMC TAN TRA WAG7 WERF WMF WROC TOTAL

TL-208

TL-209

U.232

U.233

U-234

U-235

U.236

U-237

U.238

Y-88

$Y-90$

Y -91

ZN-65

ZR-93

ZR-95

TOTAL

\begin{tabular}{|c|c|c|c|c|c|c|c|c|c|c|c|c|c|c|c|c|c|c|c|}
\hline ANLW & $\mathrm{CF}$ & $\mathrm{D}+\mathrm{D}$ & INTEC & ISU & KAPCT & KAPNY & LOS & NNS & $\mathrm{NRF}$ & $\mathrm{PBF}$ & PHNSY & PSNSY & SMC & TAN & TRA WAG7 & WERF & WMF & WROC & TOTAL \\
\hline & $<1$ & & & & & & & & & $<1$ & & & & & & $<1$ & & & $<1$ \\
\hline & & & & & & & & & & & & & & & & $<1$ & & & $<1$ \\
\hline & & & & & & & & & $<1$ & & & & $<1$ & & & $<1$ & & & $<1$ \\
\hline & $<1$ & $<1$ & & & & $<1$ & $<1$ & & $<1$ & $<1$ & & & $<1$ & $<1$ & $<1$ & $<1$ & & $<1$ & $<1$ \\
\hline$<1$ & $<1$ & $<1$ & & & & $<1$ & $<1$ & & $<1$ & $<1$ & & & $<1$ & $<1$ & $<1$ & $<1$ & $<1$ & $<1$ & $<1$ \\
\hline$<1$ & & $<1$ & & & & & & & & $<1$ & & & $<1$ & & $<1$ & $<1$ & & & $<1$ \\
\hline$<1$ & $<1$ & $<1$ & & & & $<1$ & $<1$ & & $<1$ & $<1$ & & & $<1$ & $<1$ & $<1$ & $<1$ & $<1$ & $<1$ & $<1$ \\
\hline & $<1$ & & & & & & & & & & & & & & & $<1$ & & & $<1$ \\
\hline$<1$ & $<1$ & & $<1$ & $<1$ & & $<1$ & $<1$ & & $<1$ & $<1$ & $<1$ & $<1$ & & $<1$ & $<1$ & 3 & & & 4 \\
\hline$<1$ & & & & & & & & & & & & & & & & $<1$ & & & $<1$ \\
\hline & $<1$ & & & & $<1$ & & & & $<1$ & $<1$ & & & & & $<1$ & $<1$ & & & $<1$ \\
\hline
\end{tabular}


INEEL-78 
Argonne National Laboratory-West 1998 Detail Graphs

Argonne- National Laboratory-West (ANLW) Bar Graphs

of Annual Data by Month. INEEL-81 


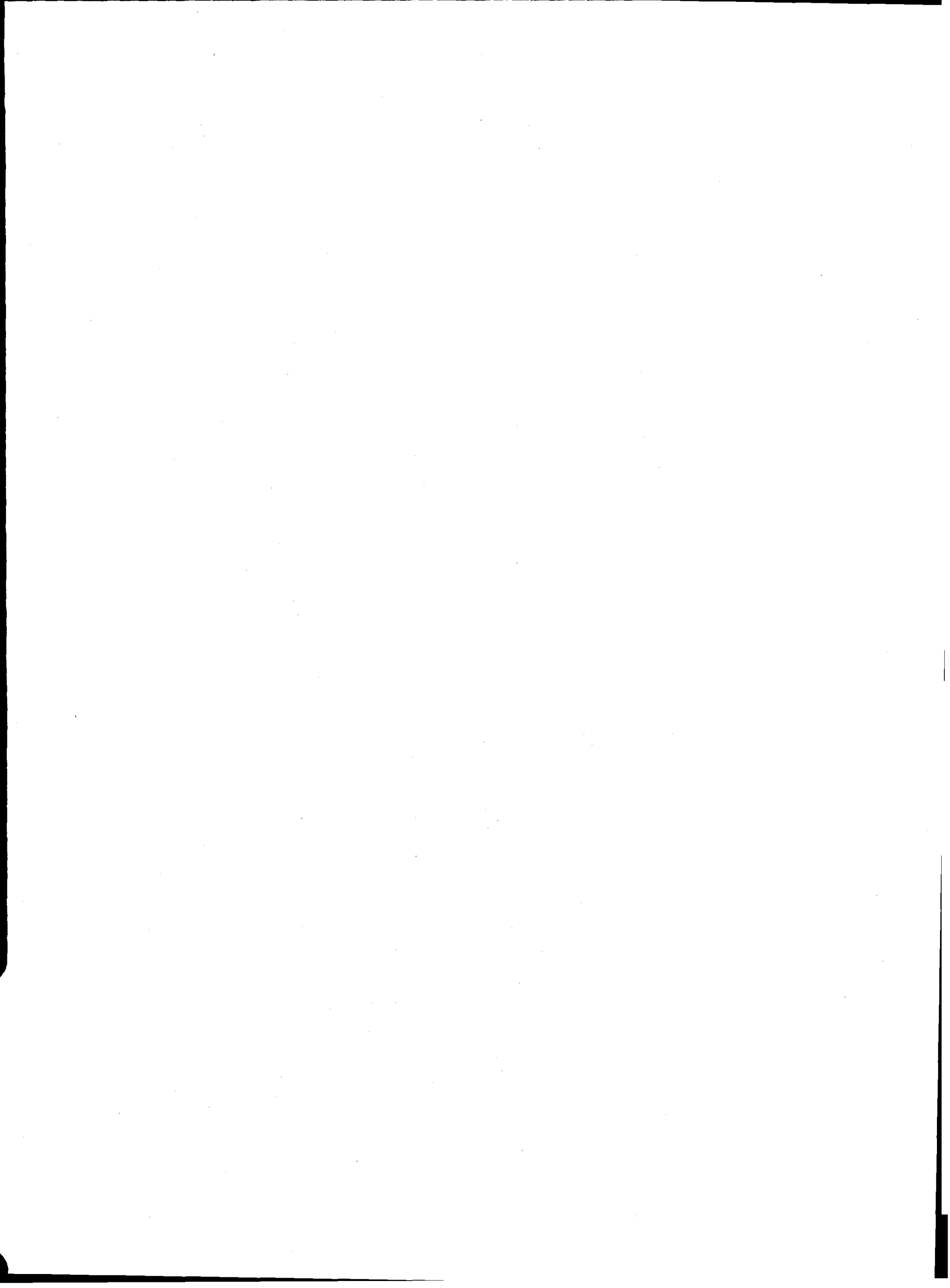



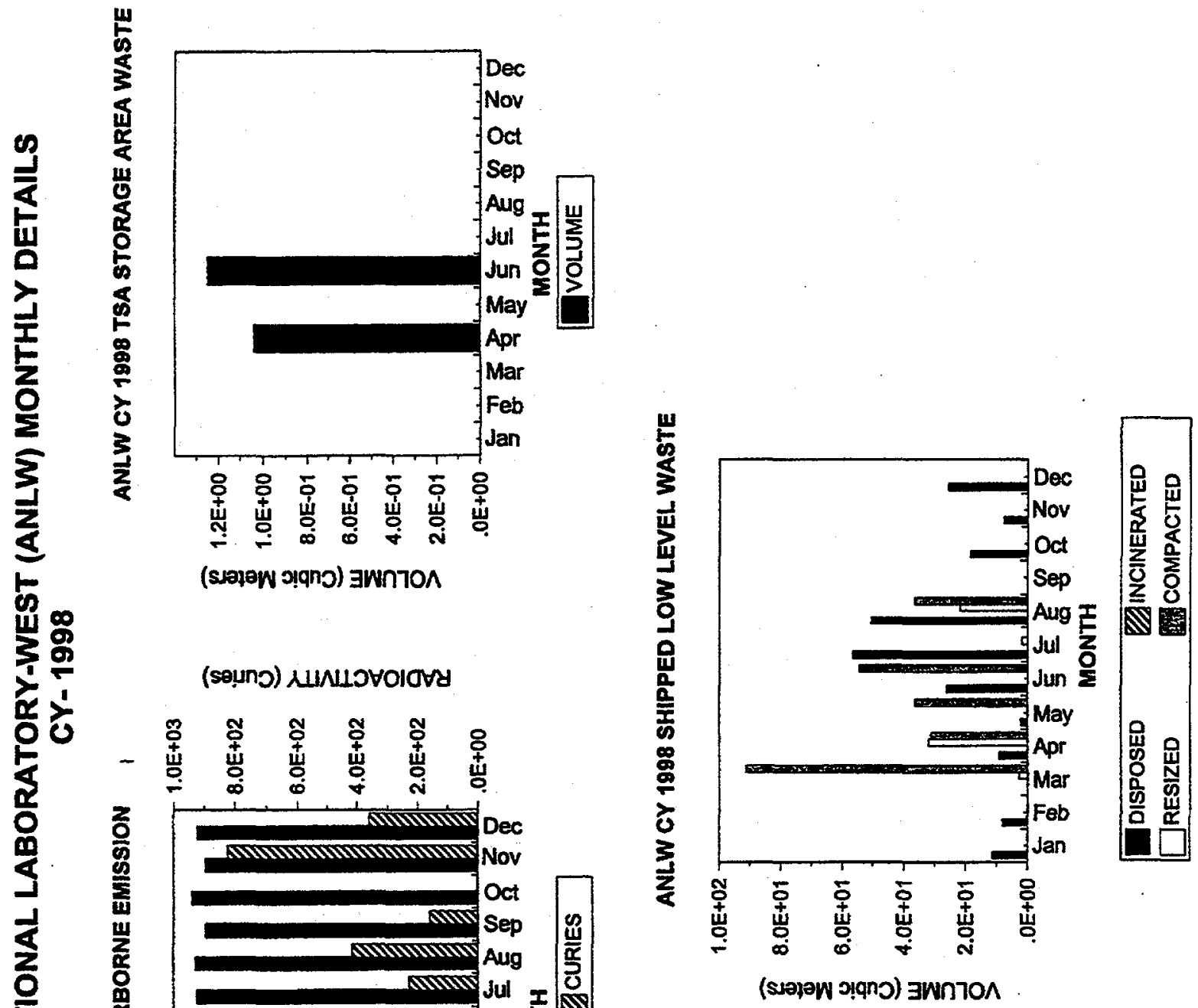

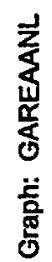


Central Facilities Area 1998 Detail Graphs

Central Facilities Area (CFA) Bar Graphs of Annual Data by Month ............................................INEEL-85 


\section{CENTRAL FACILITY AREA (CF) MONTHLY DETAILS CY-1998}

\section{CF CY 1998 SHIPPED LOW LEVEL WASTE}

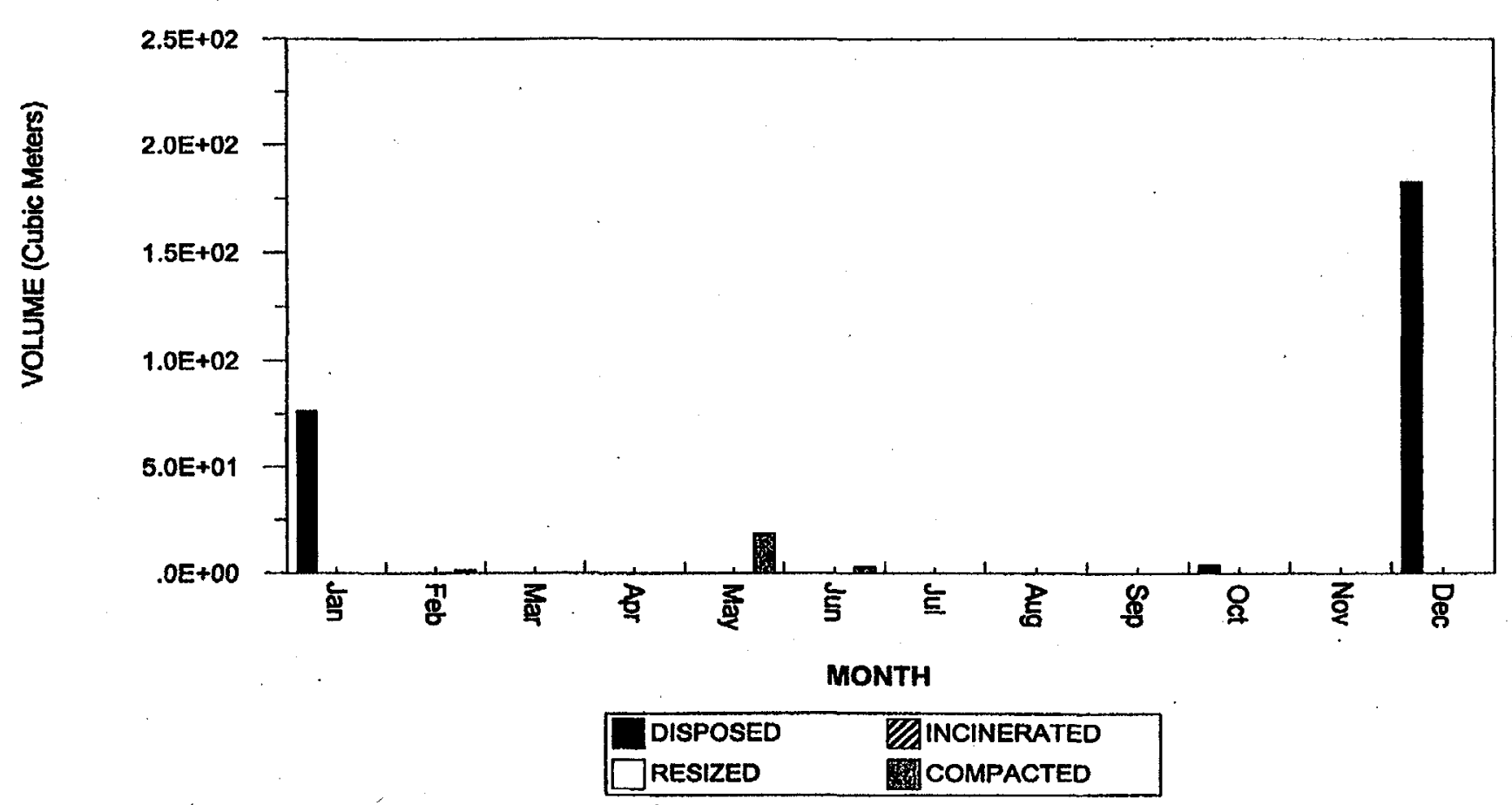


Idaho Nuclear Technology and Engineering Center (INTEC) 1998 Detail Graphs

Idaho Nuclear Technology and Engineering Center (INTEC)

Bar Graphs of Annual Data by Month.

NEEL-89 


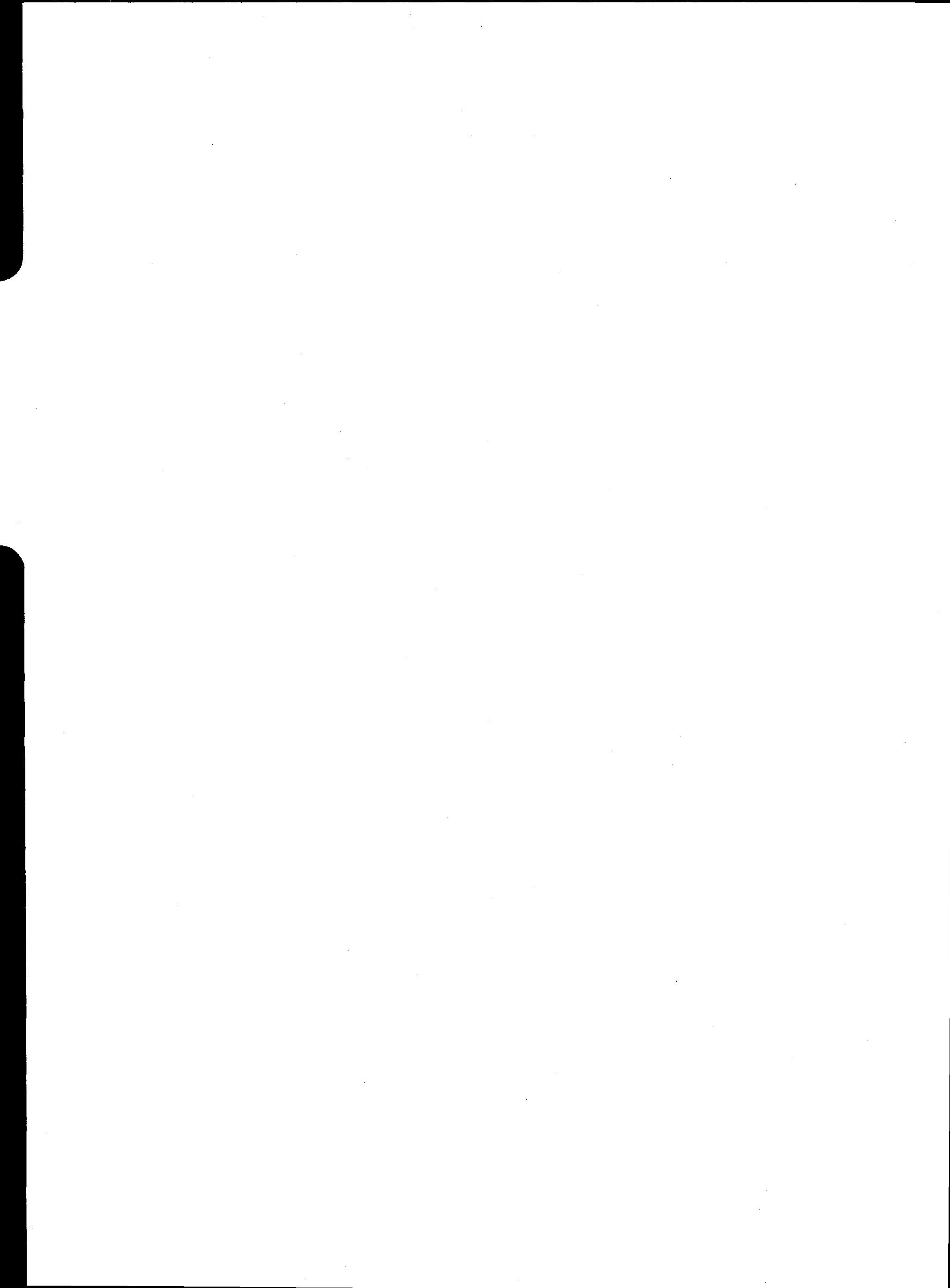




\section{IDAHO NUCLEAR TECHNOLOGY and ENGINEERING CENTER (INTEC) MONTHLY DETAILS}

CY- 1998
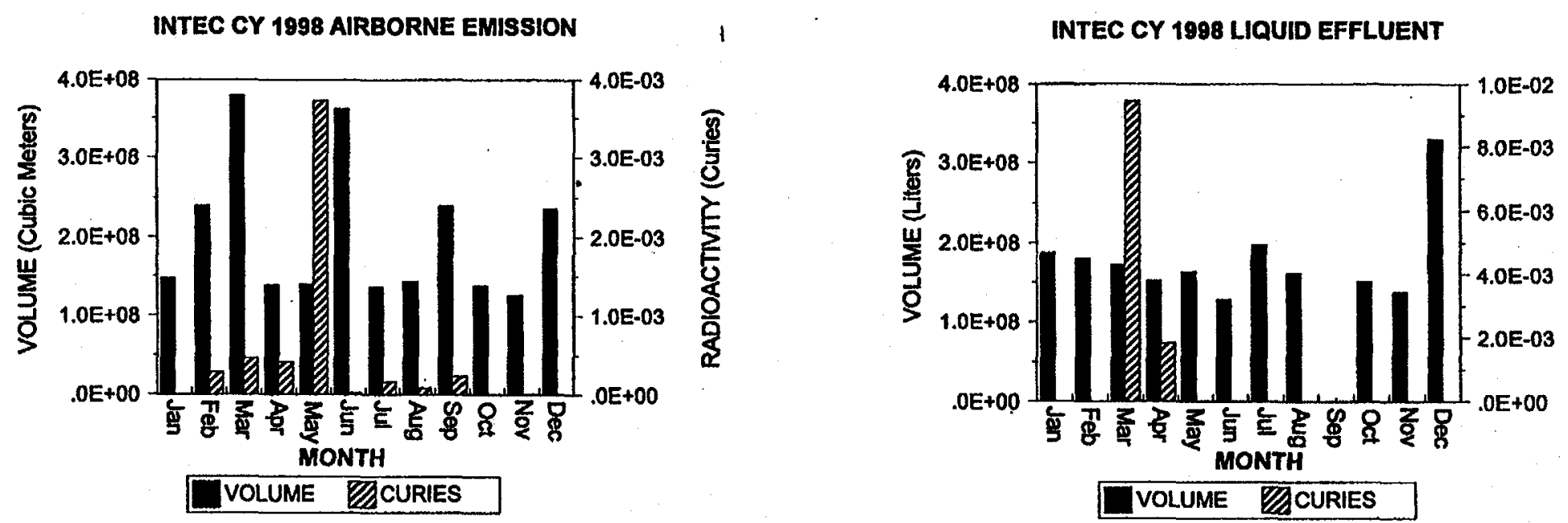

INTEC CY 1998 SHIPPED LOW LEVEL WASTE

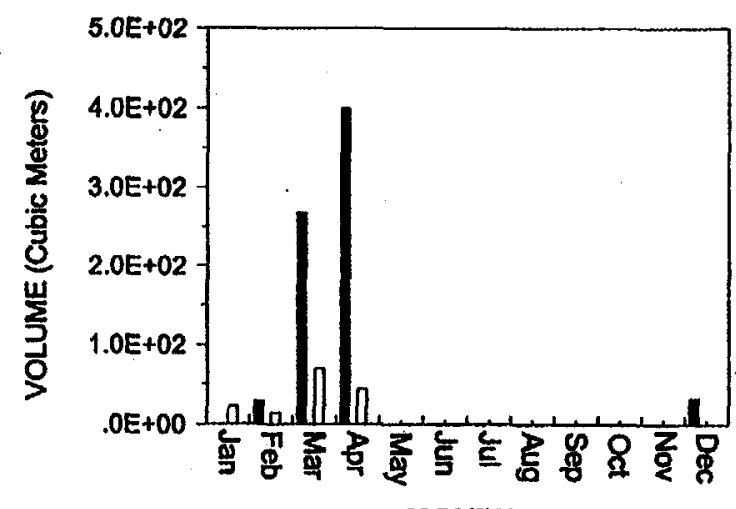

Graph: GAREAINTEC

MONTH

\begin{tabular}{|ll|}
\hline DISPOSED & GINCINERATED \\
$\square$ RESIZED & MCOMPACTED \\
\hline
\end{tabular}


INEEL-90 
Decontamination and Decommissioning 1998 Detail Graphs

Decontamination and Decommissioning (D\&D) Bar Graphs

of Annual Data by Month

INEEL-93 


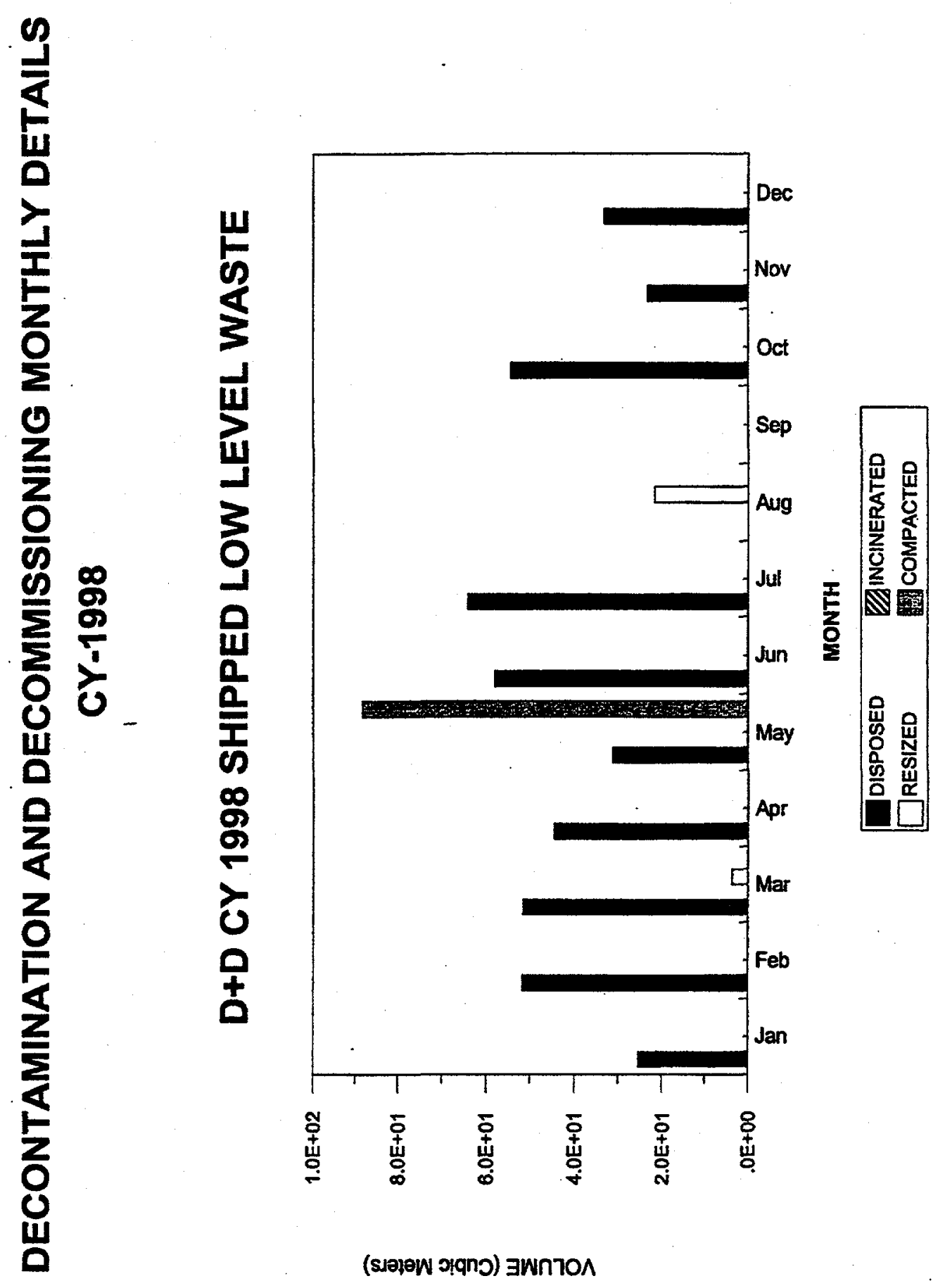

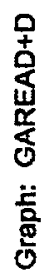


INEEL-94 
Naval Reactor Facility 1998 Detail Graphs

Naval Reactor Facility (NRF) Bar Graphs of Annual Data by Month...................................... INEEL-97 


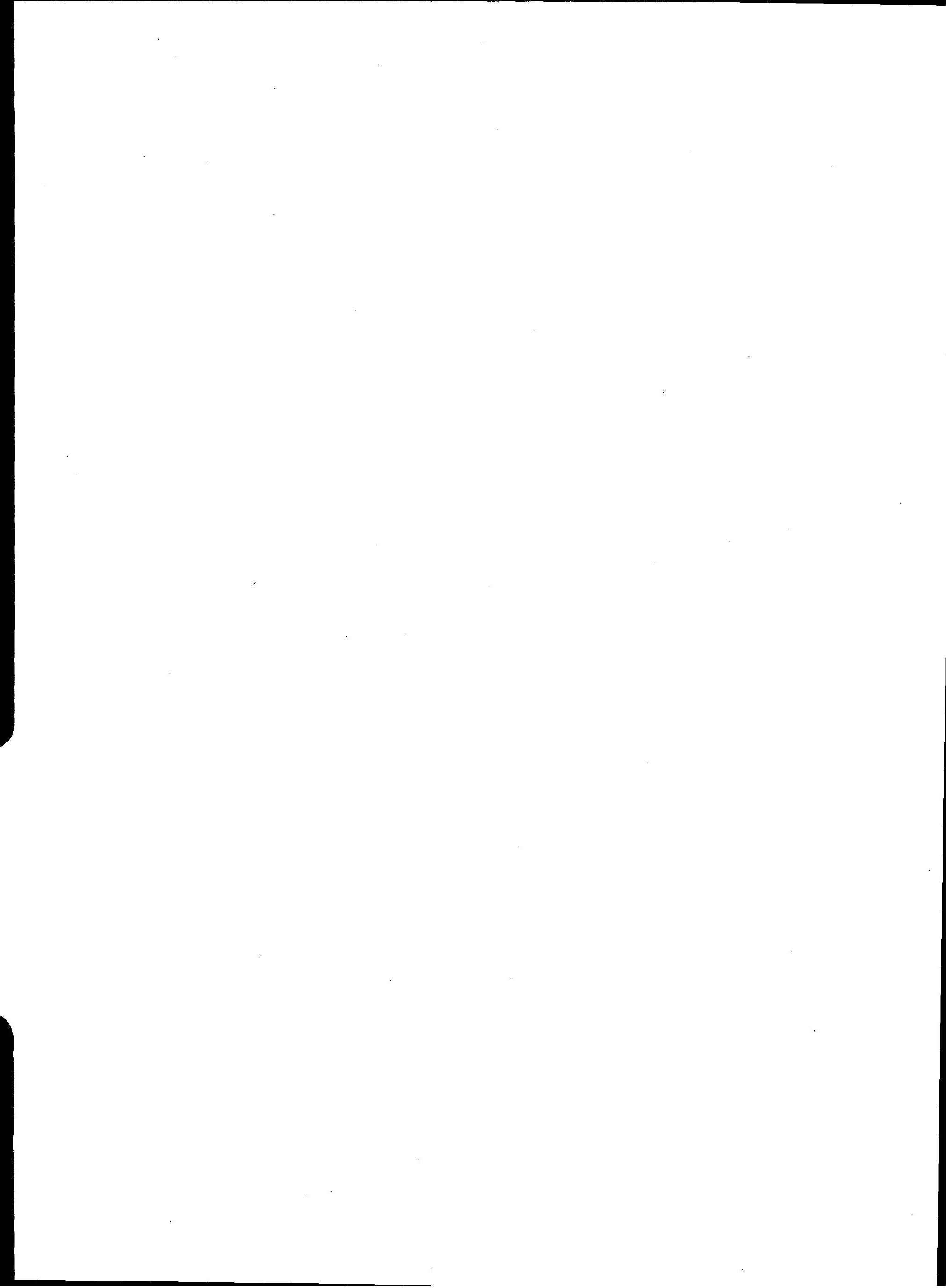




\section{NAVAL REACTORS FACILITY (NRF) MONTHLY DETAILS}

CY- 1998

NRF CY 1998 AIRBORNE EMISSION

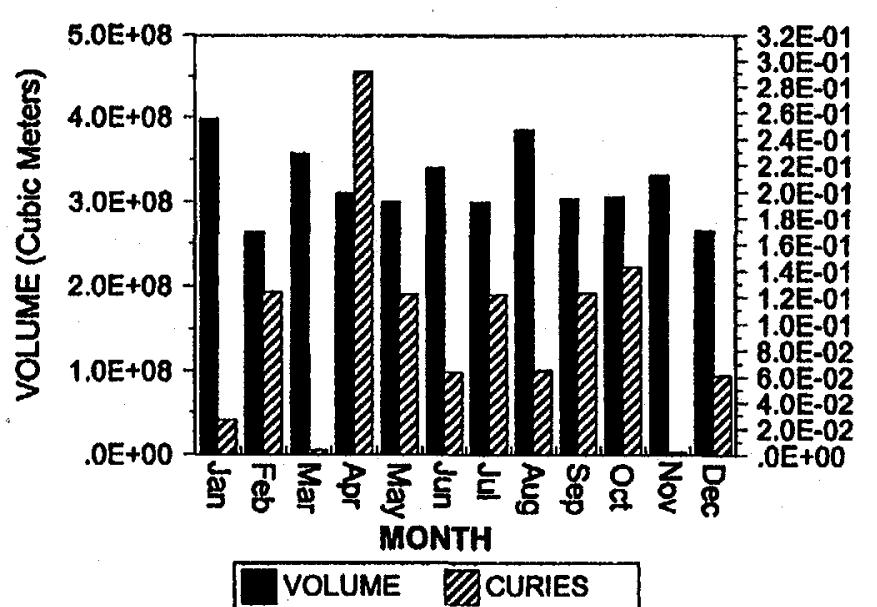

NRF CY 1998 SHIPPED LOW LEVEL WASTE

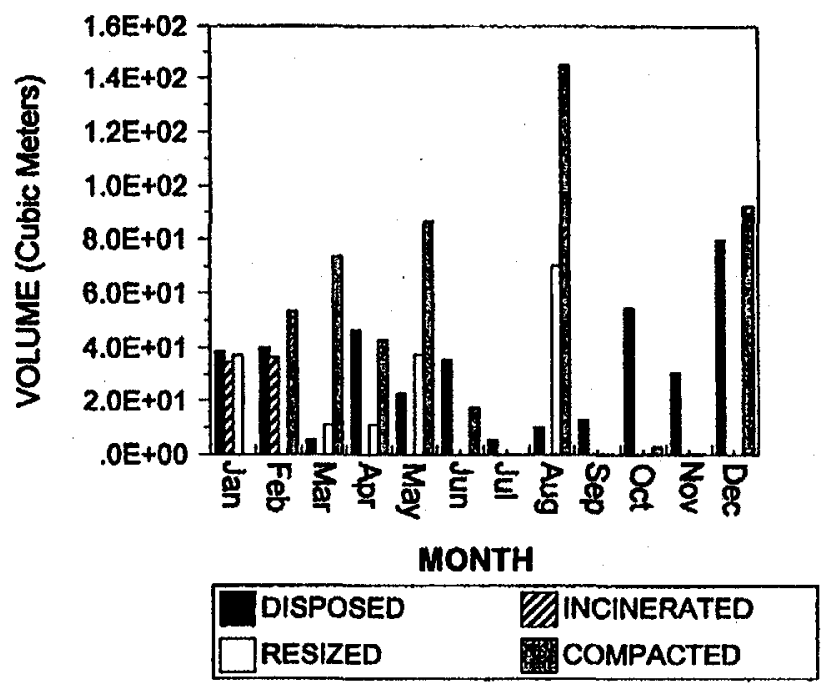
COMPACTED \$

త్ $\xi$ 


\section{Power Burst Facility 1998 Detail Graphs}

Power Burst Facility (PBF) Bar Graphs of Annual Data by Month INEEL-101 


\section{POWER BURST FACILITY (PBF) MONTHLY DETAILS}

CY- 1998

PBF CY 1998 AIRBORNE EMISSION

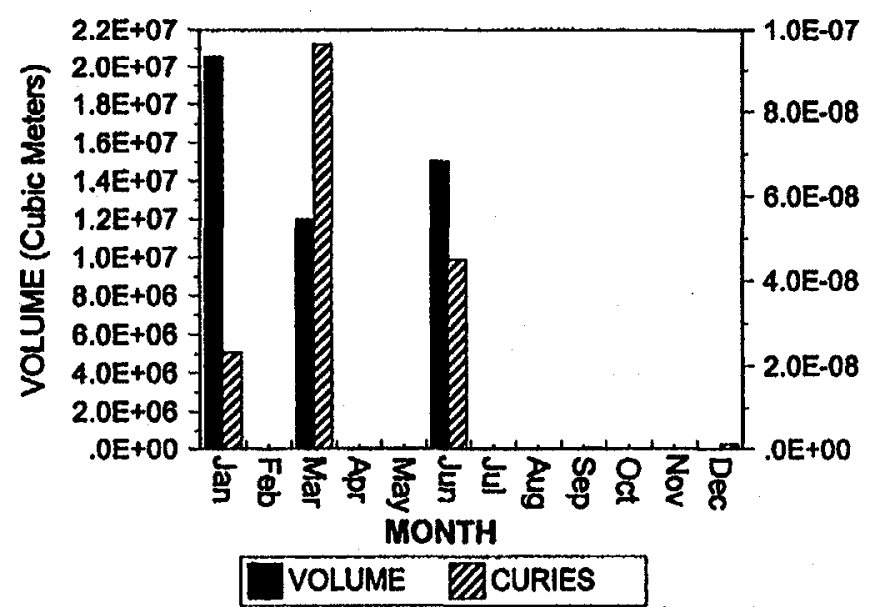

PBF CY 1998 SHIPPED LOW LEVEL WASTE

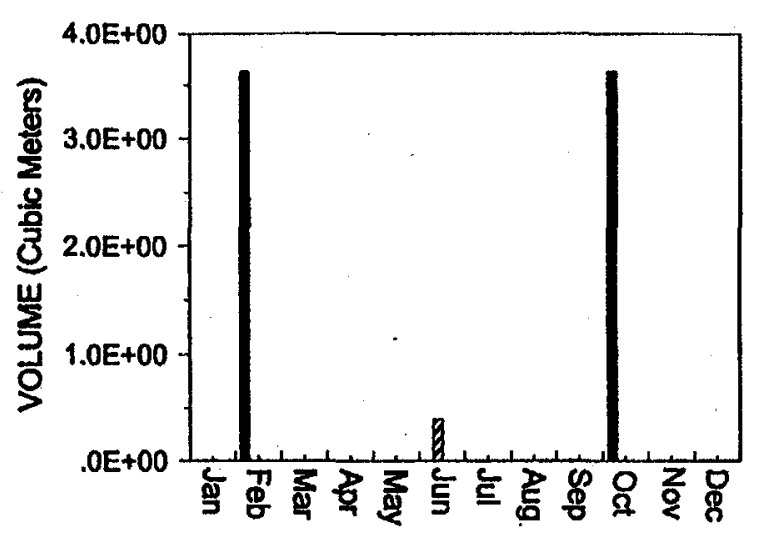

MONTH

$\begin{array}{ll}\text { DISPOSED } & \text { GINCINERATED } \\ \text { REESIZED } & \text { COMPACTED }\end{array}$


Special Manufacturing Capability 1998 Detail Graphs

Special Manufacturing Capability (SMC) Bar Graphs of

Annual Data by Month.

INEEL-105 

SPECIAL MANUFACTURING CAPABILITY (SMC) MONTHLY DETAILS CY- 1998
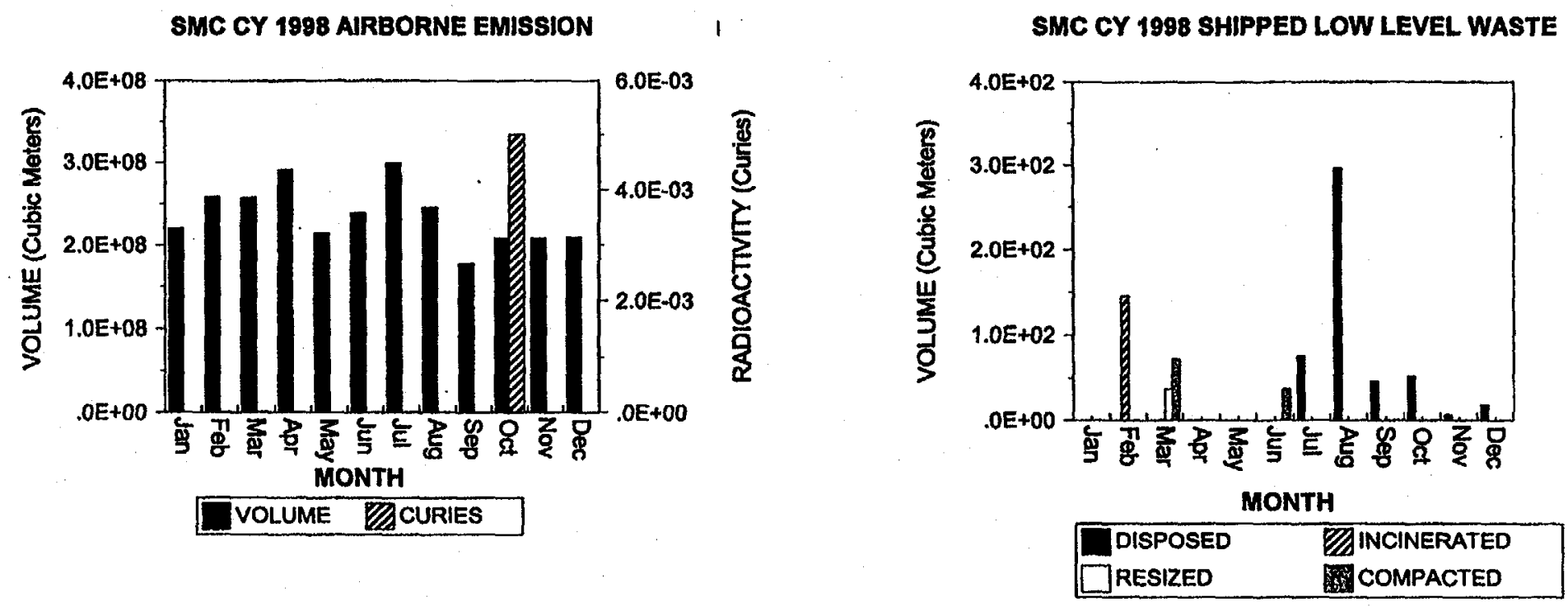

Graph: GAREASMC 


\section{Test Area North 1998 Detail Graphs}

Test Area North (TAN) Bar Graphs of Annual Data by Month ...................................................NEEL-109 


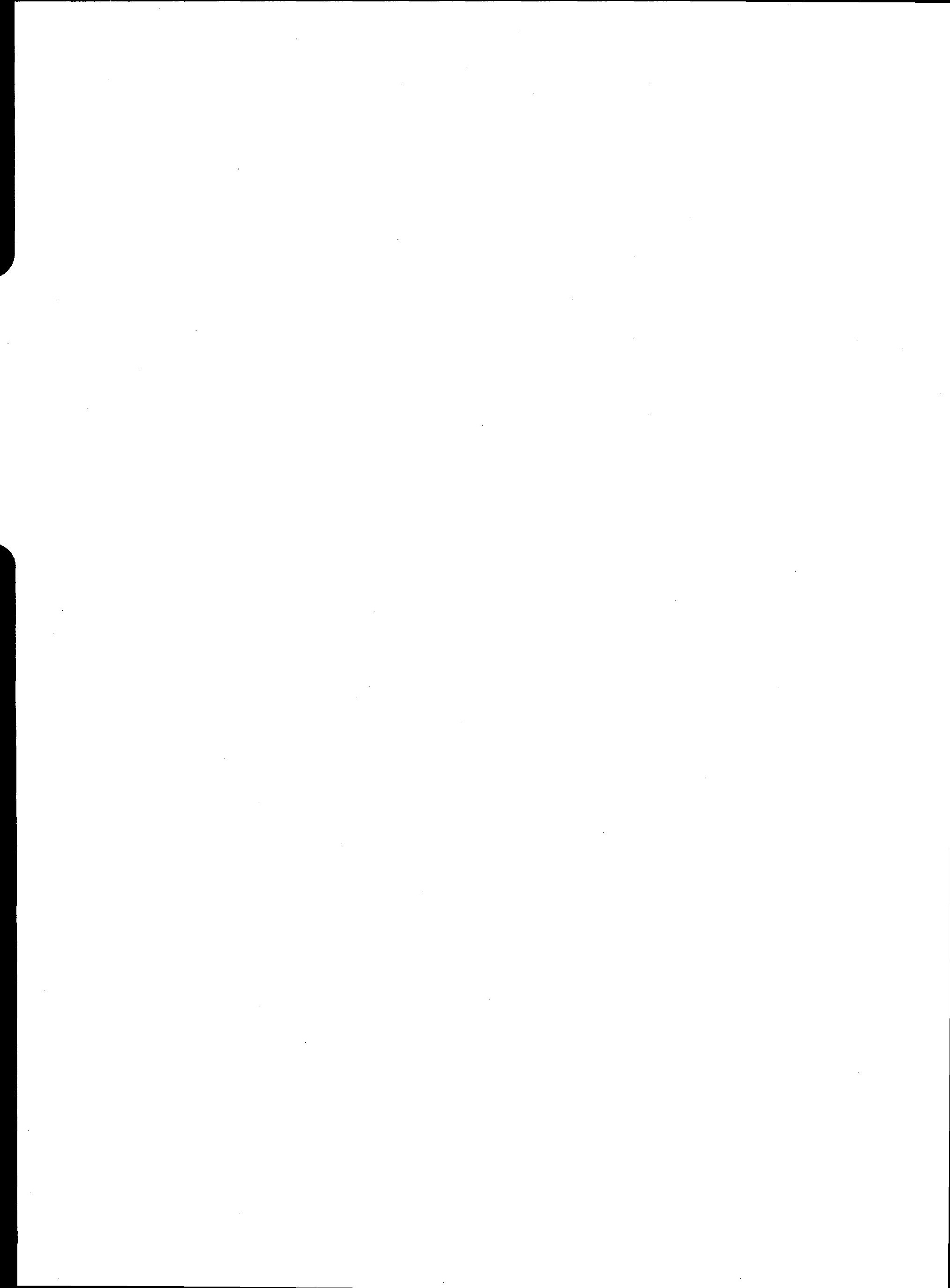


TAN CY 1998 AIRBORNE EMISSION

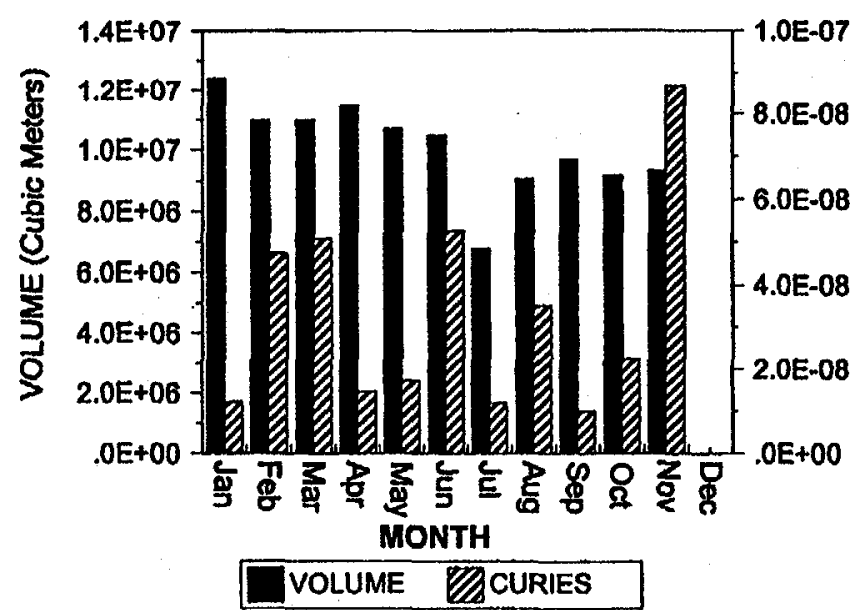

TAN CY 1998 SHIPPED LOW LEVEL WASTE

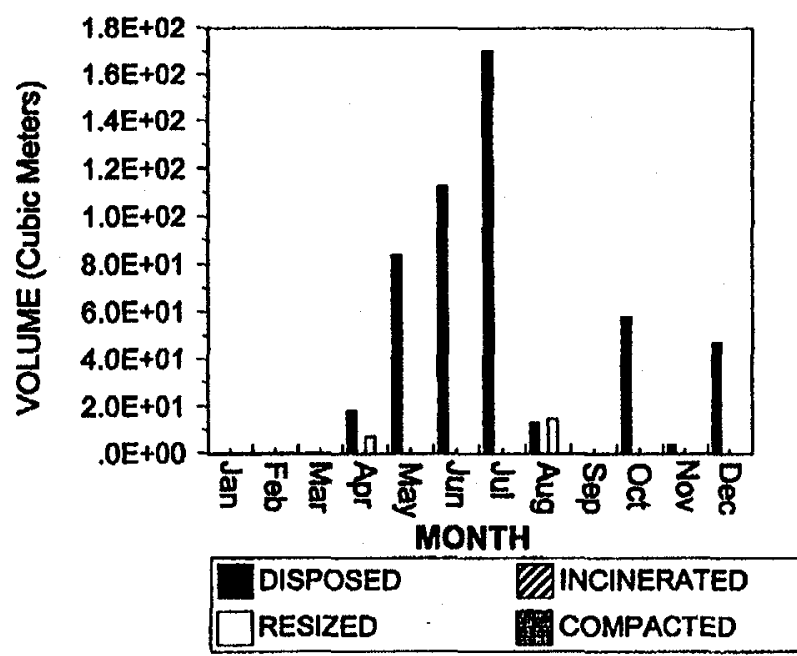

1

焉 
Test Reactor Area 1998 Detail Graphs

Test Reactor Area (TRA) Bar Graphs of Annual Data by Month INEEL-113 


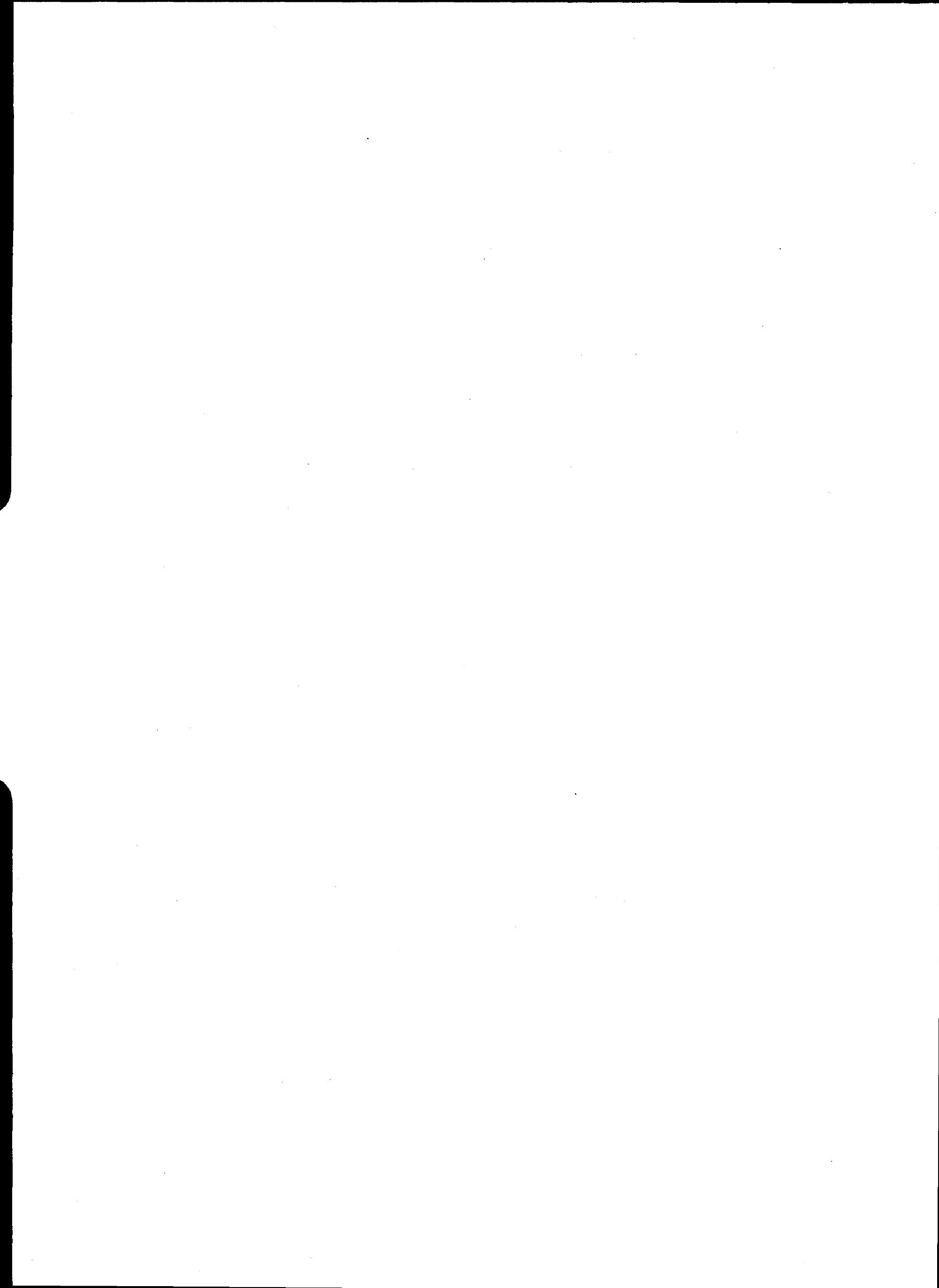



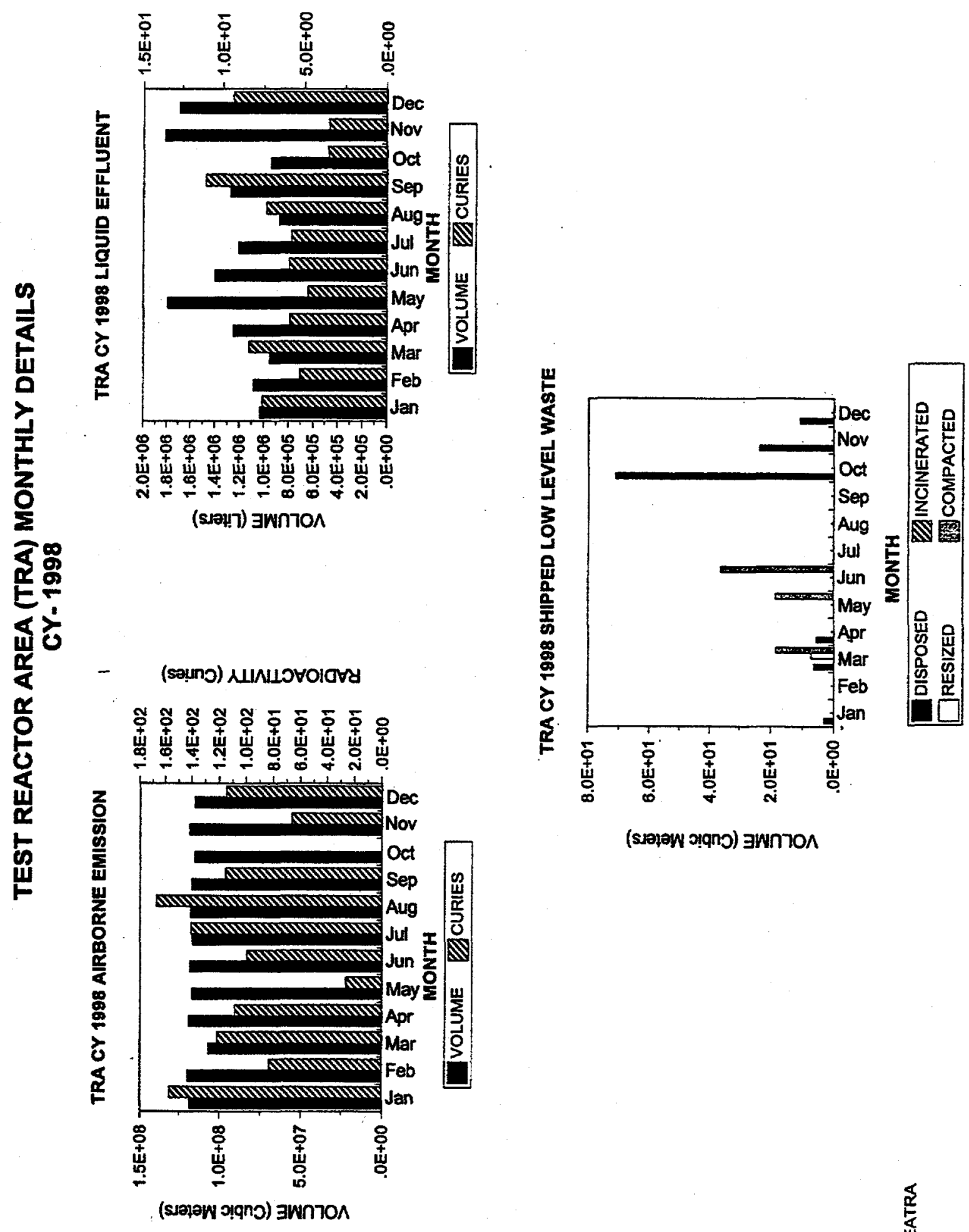

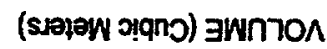


INEEL-114 


\section{Waste Area Groups 1998 Detail Graphs}

Waste Area Group 1 Bar Graphs of Annual Data by Month

INEEL-117

Waste Area Group 3 Bar Graphs of Annual Data by Month

INEEL-118

Waste Area Group 7 Bar Graphs of Annual Data by Month

INEEL-119 


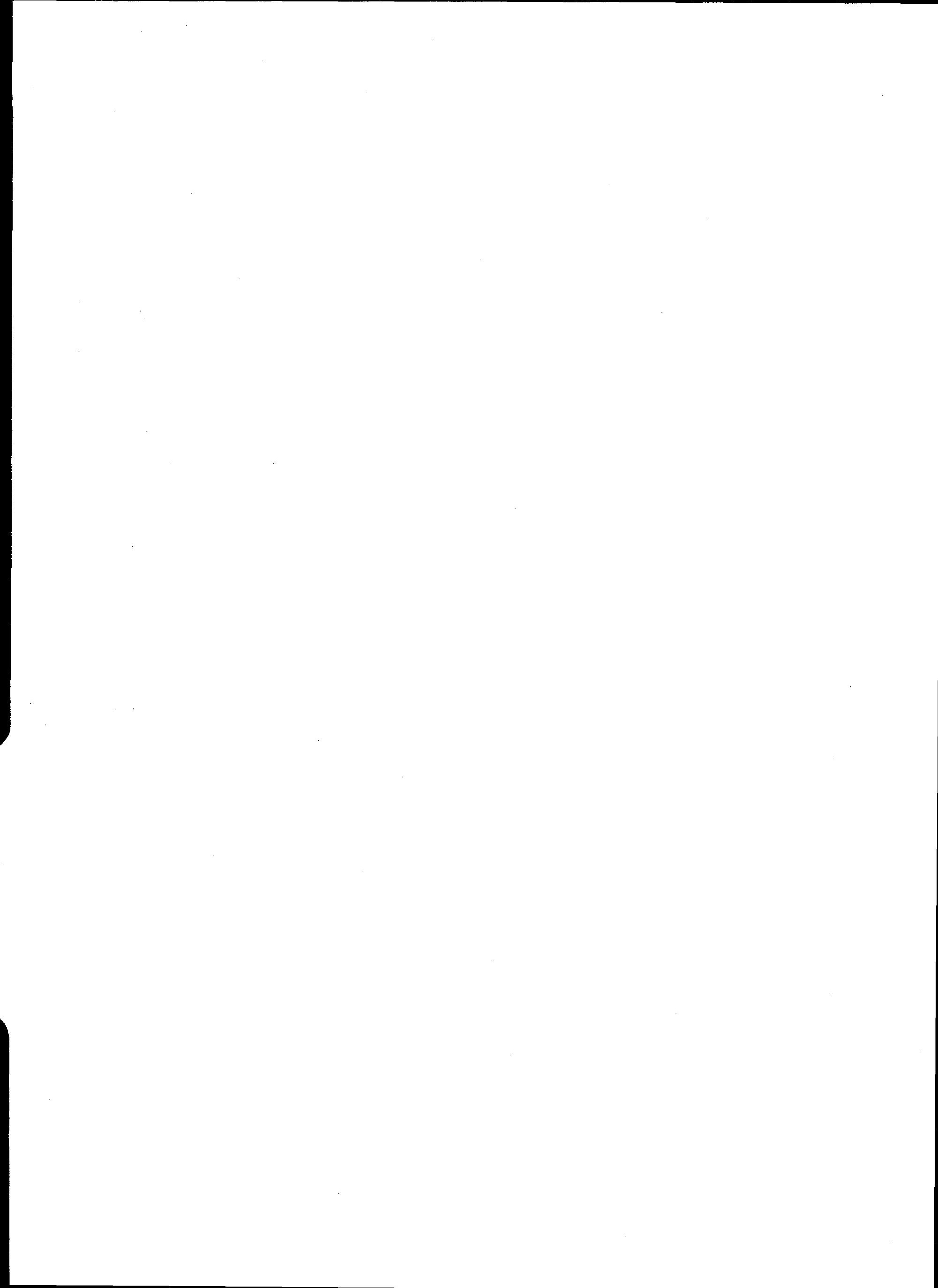


WAG1 AREA MONTHLY DETAILS CY-1998

1

WAG1 CY 1998 SHIPPED LOW LEVEL WASTE

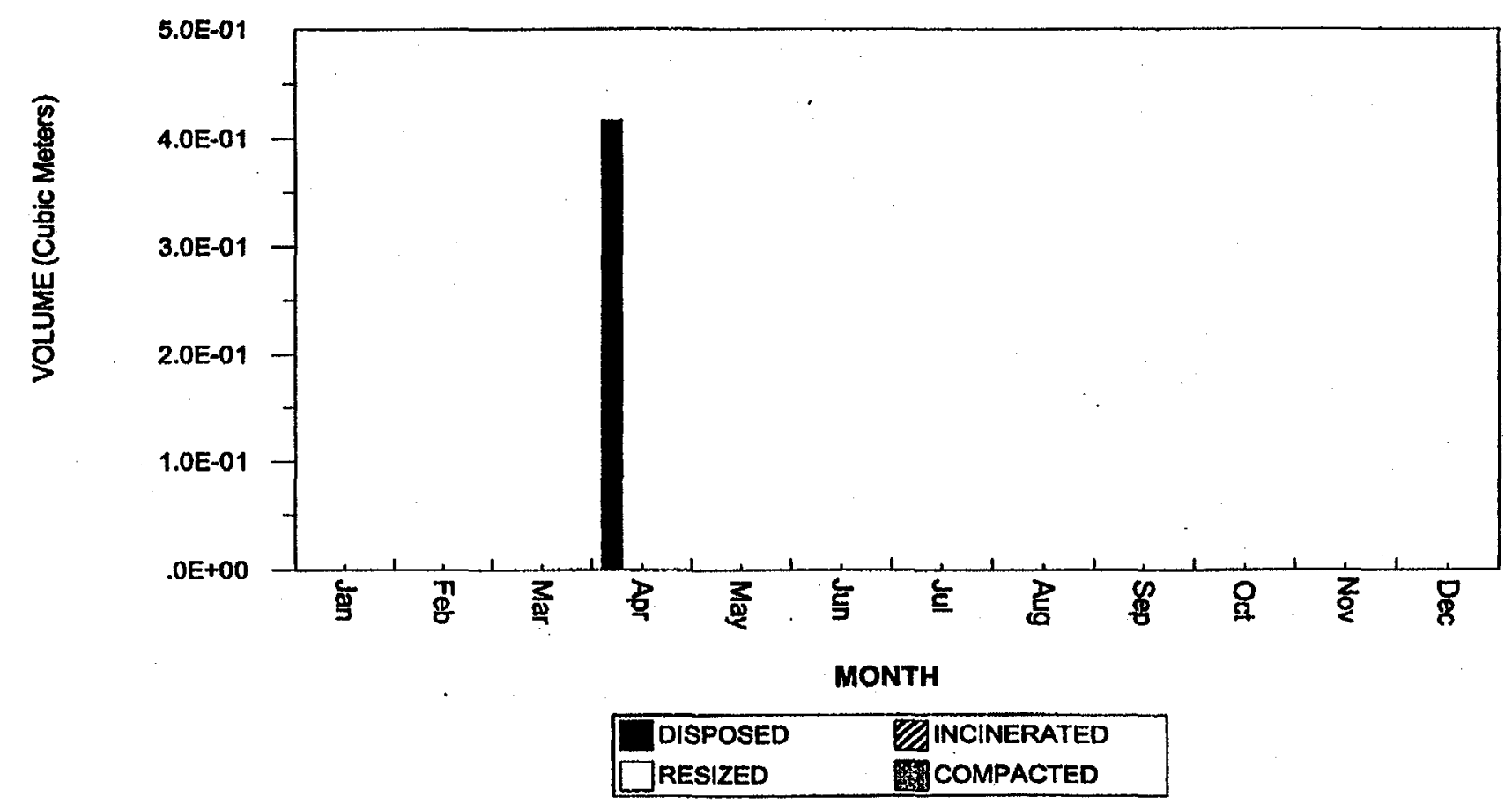




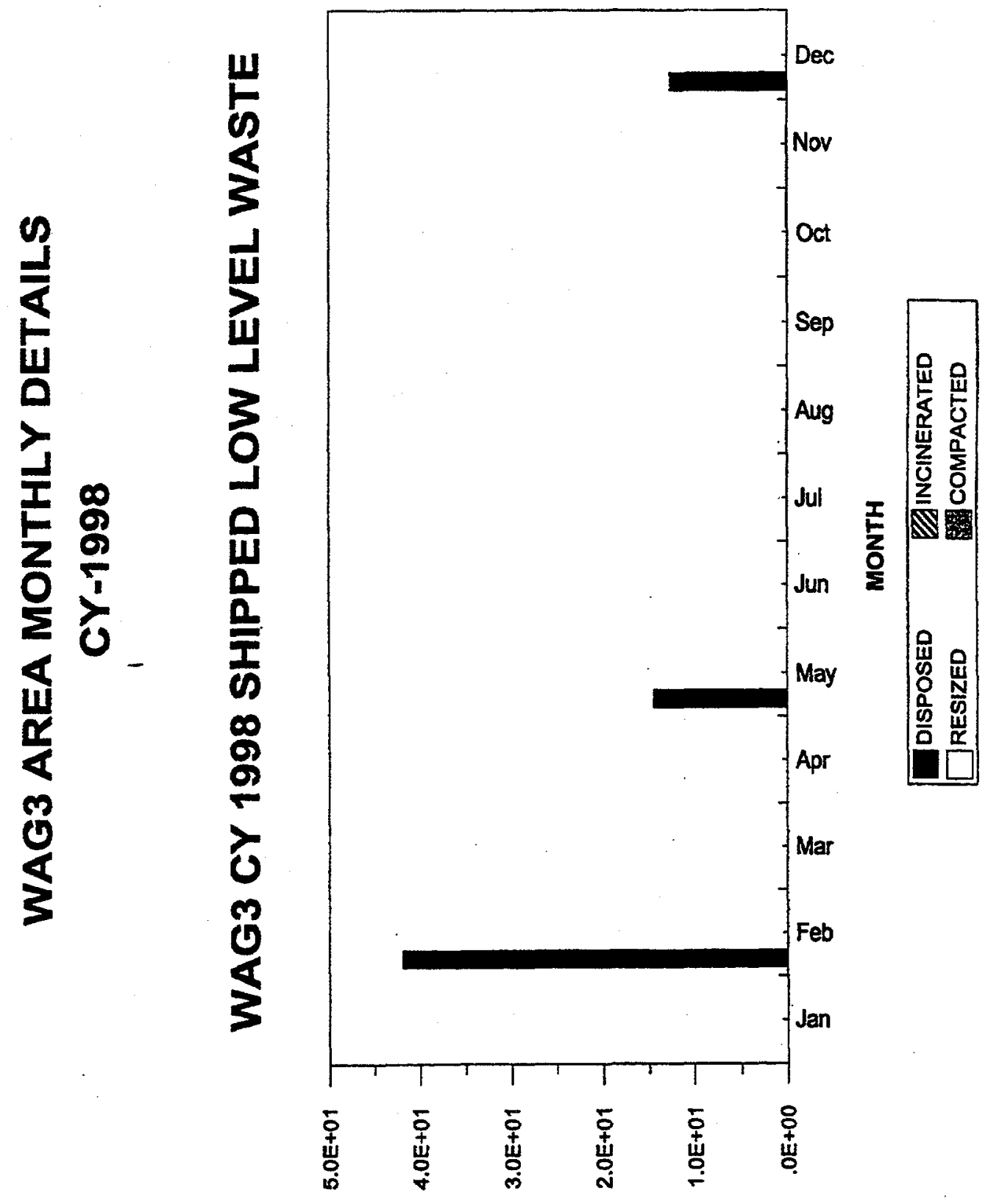

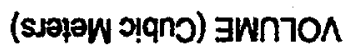




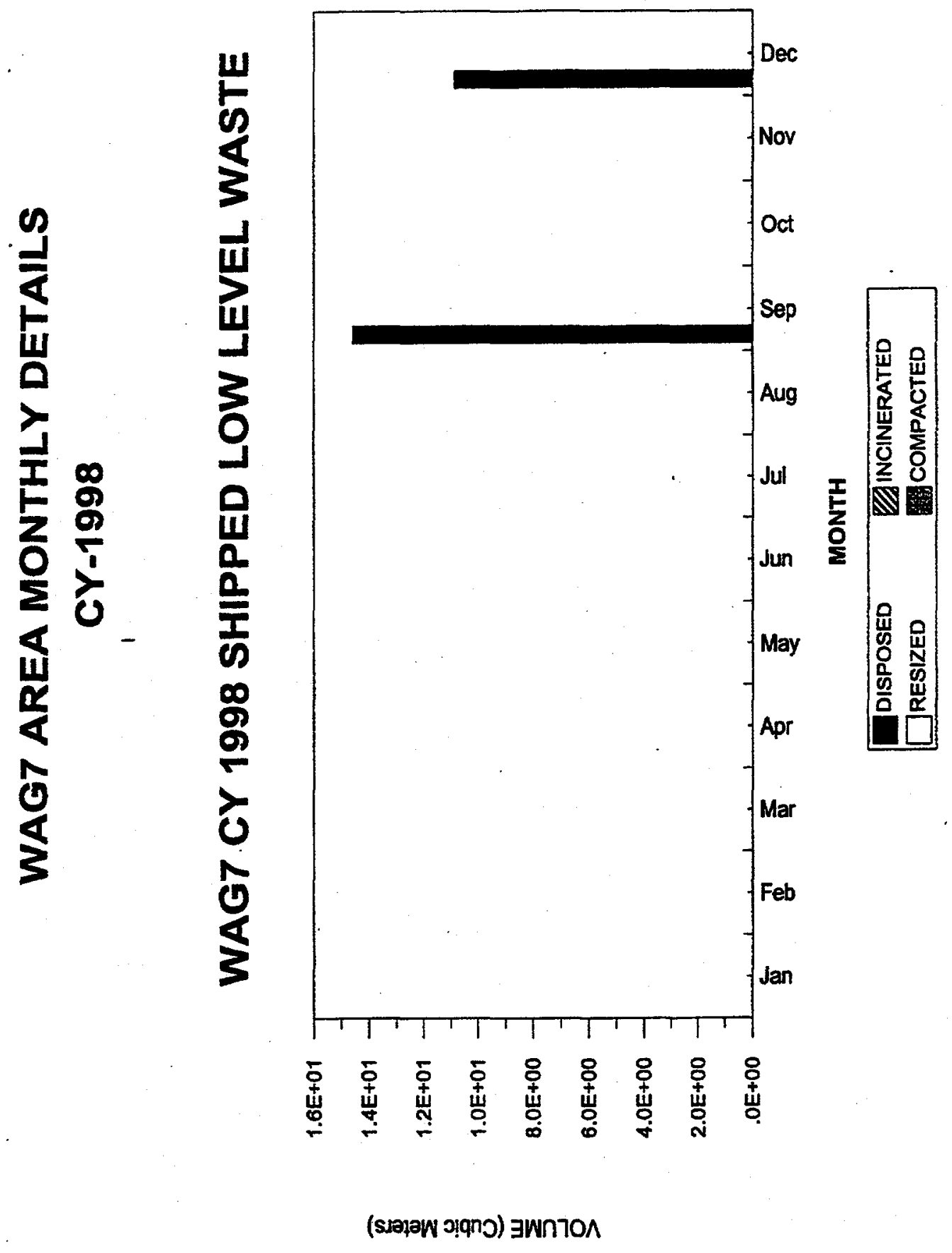

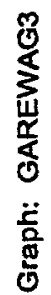


INEEL-120 


\section{Waste Experimental Reduction Facility 1998 Detail Graphs}

Waste Experimental Reduction Facility (WERF) Bar Graphs

of Annual Data by Month.

..INEEL-123 
WASTE EXPERIMENTAL REDUCTION FACILITY (WERF) MONTHLY DETAILS

CY- 1998

WERF CY 1998 AIRBORNE EMISSION

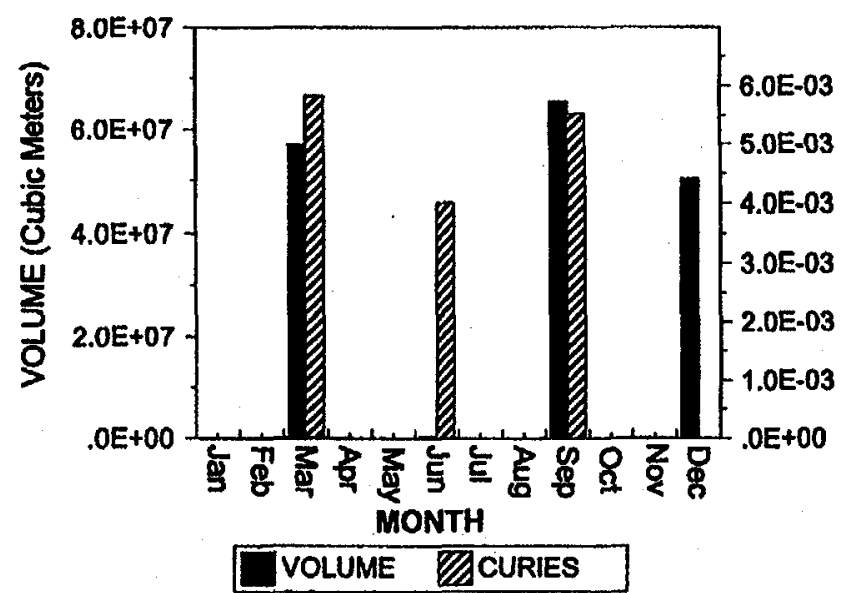

WERF CY 1998 RECEIVED/DISPOSED CONTAINERIZED WASTE

㺼

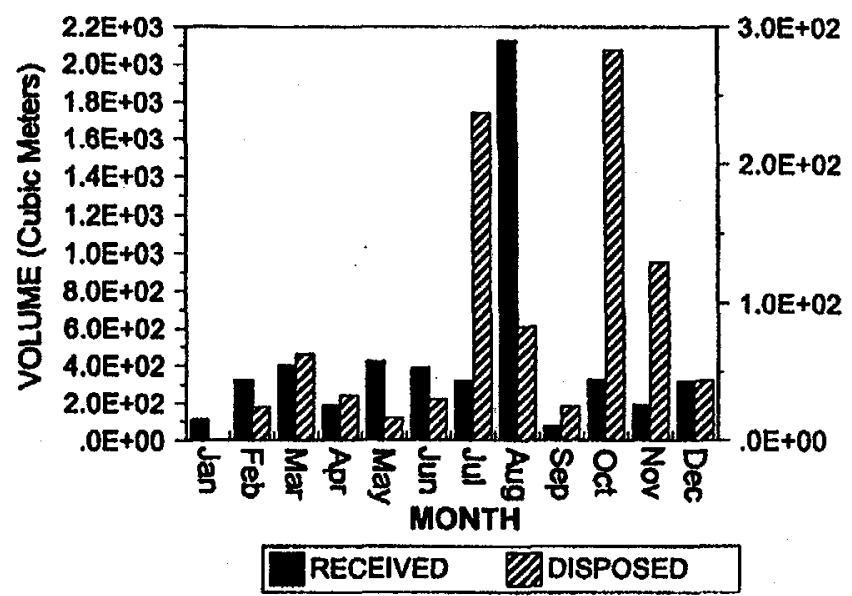


INEEL-124 
Waste Reduction Operations Complex 1998 Detail Graphs

Waste Reduction Operations Complex (WROC) Bar Graphs of Annual Data by Month. INEEL-127 


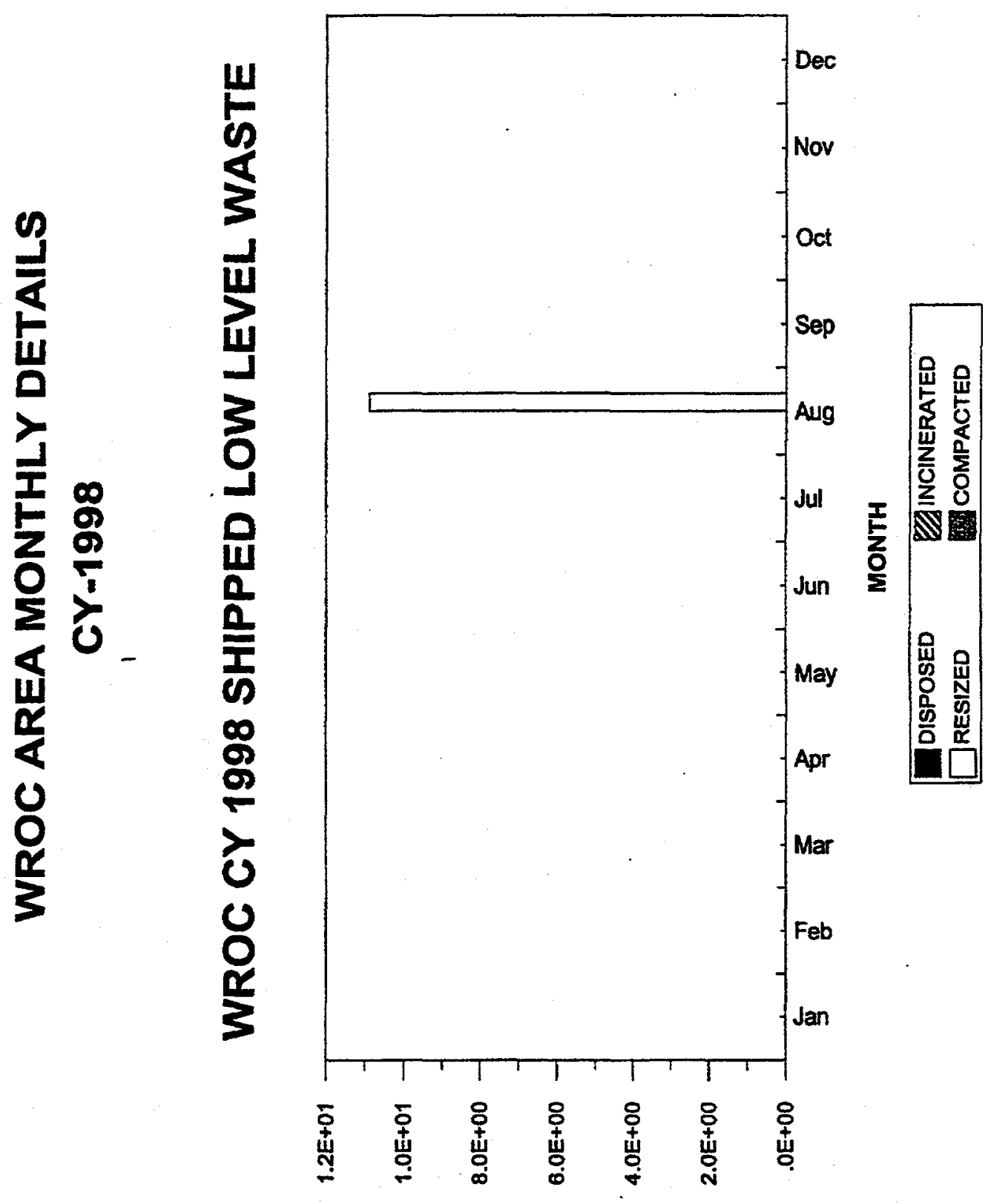

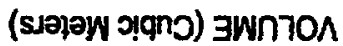


INEEL-128 


\section{Engineered Release Points}

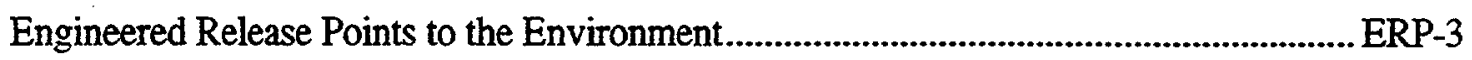


Table 1. Engineered release points to the environment.

\begin{tabular}{|c|c|c|c|c|}
\hline $\begin{array}{c}\text { Type of } \\
\text { Discharge }\end{array}$ & Area/Location & $\begin{array}{l}\text { Type of Description of } \\
\text { Discharge Surface } \\
\end{array}$ & $\begin{array}{c}\text { Air Emission } \\
\text { Inventory Stack } \\
\quad \text { No. } \\
\end{array}$ & $\begin{array}{c}\text { Active } \\
\text { (Yes/No) }\end{array}$ \\
\hline \multirow[t]{17}{*}{ Airborne } & $\begin{array}{l}\text { ANL-W/EBR-II, } \\
\text { FCF }\end{array}$ & $\begin{array}{l}\text { 61-m glass-coated steel stack, } 30 \mathrm{~m}^{3} / \mathrm{s} \mathrm{discharge} \\
\text { capacity, continuously monitored. }\end{array}$ & ANL-764-001 & Yes \\
\hline & ANL-W/FASB & $\begin{array}{l}\text { 10-m stack, } 6.64 \mathrm{~m}^{3} / \mathrm{s} \text { discharge capacity, } \\
\text { continuously monitored. }\end{array}$ & ANL-787-001 & Yes \\
\hline & ANL-W/FMF & $\begin{array}{l}11.1 \text {-m stack, } 4.6 \mathrm{~m}^{3} / \mathrm{s} \text { discharge capacity, } \\
\text { continuously monitored. }\end{array}$ & ANL-704-008 & Yes \\
\hline & ANL-W/HFEF & $\begin{array}{l}28.6-\mathrm{m} \text { stack, } 21.7 \mathrm{~m}^{3} / \mathrm{s} \text { discharge capacity, } \\
\text { continuously monitored. }\end{array}$ & ANL-785-018 & Yes \\
\hline & ANL-W/L\&O & $\begin{array}{l}\text { Two separate stacks-one stack is } 15.2 \mathrm{~m} \text { with } 10.9 \\
\mathrm{~m}^{3} / \mathrm{s} \text { discharge capacity. The other stack is } 11.3 \mathrm{~m} \\
\text { with } 7.8 \mathrm{~m}^{3} / \mathrm{s} \text { discharge capacity. Both stacks are } \\
\text { continuously monitored. }\end{array}$ & $\begin{array}{l}\text { ANL-752-004 } \\
\text { (MAIN) } \\
\text { ANL-752-005 } \\
\text { (NDA) }\end{array}$ & Yes \\
\hline & ANL-W/RLWTF & $\begin{array}{l}14.2-\mathrm{m} \text { stack, } 1.7 \mathrm{~m}^{3} / \mathrm{s} \text { discharge capacity, } \\
\text { continuously monitored. }\end{array}$ & ANL-798-017 & Yes \\
\hline & ANL-W/SCMS & $\begin{array}{l}14.6-\mathrm{m} \text { stack, } 4.7 \mathrm{~m}^{3} / \mathrm{s} \text { discharge capacity, } \\
\text { continuously monitored. }\end{array}$ & ANL-793-001 & Yes \\
\hline & ANL-W/SPF & $\begin{array}{l}6.9-\mathrm{m} \text { stack, } 2.1 \mathrm{~m}^{3} / \mathrm{s} \text { discharge capacity, } \\
\text { continuously monitored. This stack is presently } \\
\text { inactive. }\end{array}$ & ANL-799-010 & No \\
\hline & ANL-W/TREAT & $\begin{array}{l}42.7-\mathrm{m} \text { steel stack, } 2.8 \mathrm{~m}^{3} / \mathrm{s} \text { discharge capacity, } \\
\text { continuously monitored. }\end{array}$ & ANL-720-007 & Yes \\
\hline & ANL-W/ZPPR & $\begin{array}{l}22.9-\mathrm{m} \text { steel stack, } 2.3 \mathrm{~m}^{3} / \mathrm{s} \text { discharge capacity, } \\
\text { continuously monitored. }\end{array}$ & ANL-777-002 & Yes \\
\hline & ARA & $\begin{array}{l}9.1-\mathrm{m} \text { stack, } 1.4 \mathrm{~m}^{3} / \mathrm{s} \text { discharge capacity, } \\
\text { continuously monitored. These stacks were capped } \\
\text { in } 1989 . \text { D\&D removal scheduled for } 1996 .\end{array}$ & ARA001 & No \\
\hline & CF/West & $\begin{array}{l}\text { Two normal ventilation exhausts, respirator } \\
\text { maintenance } 0.6 \mathrm{~m}^{3} / \mathrm{s} \text { flow, sampled weekly when } \\
\text { operating. Facility placed on standby June } 1,1993 \text {. }\end{array}$ & $\begin{array}{l}\text { Drying ovens } \\
\text { CFA-617-030 } \\
\text { CFA-617-031 }\end{array}$ & No \\
\hline & CF/North & $\begin{array}{l}\text { Normal ventilation exhausts, laundry dryer, } 6.1 \mathrm{~m}^{3} / \mathrm{s} \\
\text { flow, continuously sampled when operating. }\end{array}$ & $\begin{array}{l}\text { CFA-617-010 } \\
\text { CFA-617-011 }\end{array}$ & No \\
\hline & INTEC/FAST & $\begin{array}{l}50 \text {-m stack, } 66.1 \mathrm{~m}^{3} / \mathrm{s} \text { discharge capacity, } \\
\text { continuously monitored and sampled for } \\
\text { particulates only. }\end{array}$ & CPP-767-001 & Yes \\
\hline & $\begin{array}{l}\text { INTEC/Main } \\
\text { Stack }\end{array}$ & $\begin{array}{l}76.2-\mathrm{m} \text { stack, } 84.95 \mathrm{~m}^{3} / \mathrm{s} \text { discharge capacity, } \\
\text { continuously monitored. }\end{array}$ & CPP-708-001 & Yes \\
\hline & INTEC/NWCF & $\begin{array}{l}22-\mathrm{m} \text { stack, } 51.9 \mathrm{~m}^{3} / \mathrm{s} \text { discharge capacity, } \\
\text { continuously monitored and sampled. }\end{array}$ & CPP-659-033 & Yes \\
\hline & $\begin{array}{l}\text { INTEC/RAL } \\
\text { Stack }\end{array}$ & $\begin{array}{l}14.8-\mathrm{m} \text { stack, } 8.5 \mathrm{~m}^{3} / \mathrm{s} \text { discharge capacity, } \\
\text { continuously monitored and sampled. }\end{array}$ & CPP-684-001 & Yes \\
\hline
\end{tabular}




\begin{tabular}{|c|c|c|c|c|}
\hline $\begin{array}{l}\text { Type of } \\
\text { Discharge }\end{array}$ & Area/Location & $\begin{array}{l}\text { Type of Description of } \\
\text { Discharge Surface }\end{array}$ & $\begin{array}{c}\text { Air Emission } \\
\text { Inventory Stack } \\
\text { No. } \\
\end{array}$ & $\begin{array}{c}\text { Active } \\
\text { (Yes/No) } \\
\end{array}$ \\
\hline & $\begin{array}{l}\text { CTF } \\
\text { (formerly LOF) }\end{array}$ & $\begin{array}{l}45.72-\mathrm{m} \text { stack, } 7.8 \mathrm{~m}^{3} / \mathrm{s} \text { discharge capacity, } \\
\text { continuously monitored. This facility is not } \\
\text { functioning and has no future plans to change } \\
\text { status. }\end{array}$ & TAN-725-001 & No \\
\hline & \multirow[t]{7}{*}{ NRF/A1W } & $\begin{array}{l}\text { 13.7-m stack, continuous monitoring when operated } \\
\text { during rad work }\end{array}$ & NRF-617-002 & Yes \\
\hline & & $\begin{array}{l}13.7-\mathrm{m} \text { stack, } .47 \mathrm{~m}^{3} / \mathrm{s} \text { discharge capacity, } \\
\text { continuously monitored. }\end{array}$ & NRF-617-013 & Yes \\
\hline & & $\begin{array}{l}13.7-\mathrm{m} \text { stack, } 4.7 \mathrm{~m}^{3} / \mathrm{s} \mathrm{A-RC} 4.7 \mathrm{~m}^{3} / \mathrm{s}, \mathrm{B}-\mathrm{RC} \text {; these } \\
\text { two share a stack, continuously monitored while } \\
\text { operating. }\end{array}$ & $\begin{array}{l}\text { NRF-617-020 } \\
\text { NRF-617-021 }\end{array}$ & $\begin{array}{l}\text { Yes } \\
\text { Yes }\end{array}$ \\
\hline & & $\begin{array}{l}12.2-\mathrm{m} \text { stack, } 8.9 \mathrm{~m}^{3} / \mathrm{s} \text { discharge capacity, } \\
\text { continuously monitored while operating. }\end{array}$ & NRF-616-012 & Yes \\
\hline & & 9.14-m stack, $39.6 \mathrm{~m}^{3} / \mathrm{s}$ ELT, infrequent operation. & NRF-616-039 & Yes \\
\hline & & $\begin{array}{l}\text { 17.1-m stack, } 17.1 \mathrm{~m}^{3} / \mathrm{s} \text { emergency } \mathrm{RC} \text {, infrequent } \\
\text { operation. }\end{array}$ & NRF-616A-002 & Yes \\
\hline & & $\begin{array}{l}\text { Multiple vent points treated as one source. } \\
\text { Continuous ambient monitoring. Ventilation rate } \\
\text { based on fugitive turnover from blowers on } \\
\text { temporary containment systems }\end{array}$ & NRF-616-PCMA & Yes \\
\hline & $\begin{array}{l}\text { NRF/A1W- } \\
\text { RWDS }\end{array}$ & $6.7-\mathrm{m}$ stack, $283 \mathrm{~m}^{3} / \mathrm{s}$ RWE, infrequent operation. & NRF-628-006 & Yes \\
\hline & \multirow[t]{3}{*}{ NRF/ECF } & $\begin{array}{l}18 \text { High bay roof vents, } 20.4-\mathrm{m} \text { vents, } 13900 \mathrm{~m}^{3} / \mathrm{s} \\
\text { continuously monitored. }\end{array}$ & $\begin{array}{c}\text { NRF-618-024 } \\
\text { through } \\
\text { NRF-618-029 } \\
\text { NRF-618-032 } \\
\text { through } \\
\text { NRF-618-043 } \\
\text { NRF-618-HBRV }\end{array}$ & Yes \\
\hline & & $\begin{array}{l}\text { In Oracl all vents mapped as } \\
31.1-\mathrm{m} \text { stack, } 23 \mathrm{~m}^{3} / \mathrm{s} ; \text { Stack } 1 \text { continuously } \\
\text { monitored. }\end{array}$ & NRF-618-099 & Yes \\
\hline & & $\begin{array}{l}18 \text {-m stack, } 12 \mathrm{~m}^{3} / \mathrm{s} ; \text { Stack } 2 \text { continuously } \\
\text { monitored. }\end{array}$ & NRF-618-103 & Yes \\
\hline & \multirow[t]{4}{*}{ NRF/S1W } & $45-\mathrm{m}$ stack, $2.85 \mathrm{~m}^{3} / \mathrm{s}$, fan room & NRF-601-019C & Yes \\
\hline & & $\begin{array}{l}1.3 \mathrm{~m}^{3} / \mathrm{s} \text { Chem stack shares the stack but } \\
\text { continuously monitored separately. }\end{array}$ & NRF-601-019A & Yes \\
\hline & & $\begin{array}{l}\text { 16-m stack, } 1 \mathrm{~m}^{3} / \mathrm{s} \mathrm{RC} \text { exhaust; infrequent } \\
\text { operation, monitored while operating. }\end{array}$ & NRF-601-023 & Yes \\
\hline & & $\begin{array}{l}6 \text { High bay roof vents, no active radiological work, } \\
\text { building breathing rates without any operating fans. } \\
\text { Multiple vents combined as single source }\end{array}$ & NRF-601-HBRV & Yes \\
\hline
\end{tabular}




\begin{tabular}{|c|c|c|c|c|}
\hline $\begin{array}{c}\text { Type of } \\
\text { Discharge }\end{array}$ & Area/Location & $\begin{array}{l}\text { Type of Description of } \\
\text { Discharge Surface }\end{array}$ & $\begin{array}{c}\text { Air Emission } \\
\text { Inventory Stack } \\
\text { No. } \\
\end{array}$ & $\begin{array}{c}\text { Active } \\
\text { (Yes/No) }\end{array}$ \\
\hline & NRF/S5G & $\begin{array}{l}26-\mathrm{m} \text { stack, } 12.5 \mathrm{~m}^{3} / \mathrm{s} \text { RAVE; continuously } \\
\text { operating, continuously monitored. }\end{array}$ & NRF-633A-057 & Yes \\
\hline & & $\begin{array}{l}6 \text { High bay rood vents treated as one source. } \\
\text { Continuous ambient monitoring. Building } \\
\text { breathing rate without any operating fans. }\end{array}$ & NRF-633-HBRV & Yes \\
\hline . & NRF/MSC & Miscellaneous and fugitive sources, ground level. & NRF-MSC-MSC & Yes \\
\hline & PBF & $\begin{array}{l}24.4-\mathrm{m} \text { stack, } 2.8 \mathrm{~m}^{3} / \mathrm{s} \text { discharge capacity, } \\
\text { continuously monitored. }\end{array}$ & PER 620-016 & Yes \\
\hline & $\begin{array}{l}\text { SMC/R\&D } \\
\text { Process }\end{array}$ & $\begin{array}{l}17.7-\mathrm{m} \text { stack, } 23.6 \mathrm{~m}^{3} / \mathrm{s} \text { discharge capacity, } \\
\text { continuously monitored. }\end{array}$ & SMC-607-039 & Yes \\
\hline & $\begin{array}{l}\text { SMC/MDF QC } \\
\text { LAB }\end{array}$ & $\begin{array}{l}17.99 \text {-m stack, } 7.79 \mathrm{~m}^{3} / \mathrm{s} \text { discharge capacity, } \\
\text { continuously monitored. }\end{array}$ & SMC-607-119 & Yes \\
\hline & SMC/LINE 2a & $\begin{array}{l}\text { 14.4-m stack, } 18.12 \mathrm{~m}^{3} / \mathrm{s} \text { discharge capacity, } \\
\text { continuously monitored. }\end{array}$ & SMC-629-013 & Yes \\
\hline & SMC/LINE 2b & $\begin{array}{l}\text { 8.36-m stack, } 3.12 \mathrm{~m}^{3} / \mathrm{s} \text { discharge capacity, } \\
\text { continuously monitored. }\end{array}$ & SMC-629-014 & Yes \\
\hline & SMC/LINE 2B & $\begin{array}{l}8.36-\mathrm{m} \text { stack, } 3.12 \mathrm{~m}^{3} / \mathrm{s} \text { discharge capacity, } \\
\text { continuously monitored. }\end{array}$ & SMC-629-012 & Yes \\
\hline & $\begin{array}{l}\text { SMC } \\
\text { Process } \$ 6\end{array}$ & $\begin{array}{l}16.97-\mathrm{m} \text { stack, } 7.08 \mathrm{~m}^{3} / \mathrm{s} \text { discharge capacity, } \\
\text { continuously monitored. }\end{array}$ & SMC-679-027 & Yes \\
\hline & $\begin{array}{l}\text { SMC } \\
\text { Process S7 }\end{array}$ & $\begin{array}{l}16.97-\mathrm{m} \text { stack, } 7.08 \mathrm{~m}^{3} / \mathrm{s} \text { discharge capacity, } \\
\text { continuously monitored. }\end{array}$ & SMC-679-026 & Yes \\
\hline & $\begin{array}{l}\text { SMC } \\
\text { Process S8 }\end{array}$ & $\begin{array}{l}16.97-\mathrm{m} \text { stack, } 7.08 \mathrm{~m}^{3} / \mathrm{s} \text { discharge capacity, } \\
\text { continuously monitored. }\end{array}$ & SMC-679-025 & Yes \\
\hline & $\begin{array}{l}\text { SMC } \\
\text { Process S9 }\end{array}$ & $\begin{array}{l}16.97-\mathrm{m} \text { stack, } 7.08 \mathrm{~m}^{3} / \mathrm{s} \text { discharge capacity, } \\
\text { continuously monitored. }\end{array}$ & SMC-679-024 & Yes \\
\hline & $\begin{array}{l}\text { SMC } \\
\text { Process S10 }\end{array}$ & $\begin{array}{l}16.97-\mathrm{m} \text { stack, } 7.08 \mathrm{~m}^{3} / \mathrm{s} \text { discharge capacity, } \\
\text { continuously monitored. }\end{array}$ & SMC-679-023 & Yes \\
\hline & $\begin{array}{l}\text { SMC } \\
\text { Process S11 }\end{array}$ & $\begin{array}{l}16.97-\mathrm{m} \text { stack, } 7.08 \mathrm{~m}^{3} / \mathrm{s} \text { discharge capacity, } \\
\text { continuously monitored. }\end{array}$ & SMC-679-022 & Yes \\
\hline & $\begin{array}{l}\text { SMC/Scrap } \\
\text { Handling }\end{array}$ & $\begin{array}{l}16.97-\mathrm{m} \text { stack, } 7.93 \mathrm{~m}^{3} / \mathrm{s} \text { discharge capacity, } \\
\text { continuously monitored. }\end{array}$ & SMC-679-020 & Yes \\
\hline & $\begin{array}{l}\text { SMC/Liquid } \\
\text { Reclaim }\end{array}$ & $\begin{array}{l}16.97-\mathrm{m} \text { stack, } 7.93 \mathrm{~m}^{3} / \mathrm{s} \text { discharge capacity, } \\
\text { continuously monitored. }\end{array}$ & SMC-679-018 & Yes \\
\hline & $\begin{array}{l}\text { SMC/PRF Stack } \\
\text { S14 }\end{array}$ & $\begin{array}{l}\text { 33.54-m stack, } 0.94 \mathrm{~m}^{3} / \mathrm{s} \text { discharge capacity, } \\
\text { continuously monitored. }\end{array}$ & SMC-679-016 & No \\
\hline & TAN Decon & $\begin{array}{l}29.6-\mathrm{m} \text { stack, } 24.9 \mathrm{~m}^{3} / \mathrm{s} \text { discharge capacity, } \\
\text { continuously monitored currently no emissions. } \\
\text { Currently in process of deactivation. }\end{array}$ & TAN-607-136 & No \\
\hline
\end{tabular}




\begin{tabular}{|c|c|c|c|c|}
\hline $\begin{array}{c}\text { Type of } \\
\text { Discharge } \\
\end{array}$ & Area/Location & $\begin{array}{l}\text { Type of Description of } \\
\text { Discharge Surface }\end{array}$ & $\begin{array}{c}\text { Air Emission } \\
\text { Inventory Stack } \\
\text { No. } \\
\end{array}$ & $\begin{array}{c}\text { Active } \\
\text { (Yes/No) }\end{array}$ \\
\hline & TAN/TSF & $\begin{array}{l}51.4-\mathrm{m} \text { stack, } 7.9 \mathrm{~m}^{3} / \mathrm{s} \text { discharge capacity, } \\
\text { continuously monitored. }\end{array}$ & TAN-734-001 & Yes \\
\hline & TAN/PREPP & $\begin{array}{l}26.1-\mathrm{m} \text { stack, } 3 \mathrm{~m}^{3} / \mathrm{s} \text { discharge capacity, } \\
\text { continuously monitored. Facility was never } \\
\text { activated-currently being scheduled for } \\
\text { decommissioning. }\end{array}$ & TAN-607-107 & No \\
\hline & TRA/ATR & $\begin{array}{l}76.2-\mathrm{m} \text { stack, } 21.2 \mathrm{~m}^{3} / \mathrm{s} \text { discharge capacity, } \\
\text { continuously monitored. }\end{array}$ & TRA-770-001 & Yes \\
\hline & TRA/Chem Lab & $\begin{array}{l}29.6-\mathrm{m} \text { stack, } 6.6 \mathrm{~m}^{3} / \mathrm{s} \text { discharge capacity, } \\
\text { continuously monitored. }\end{array}$ & TRA-604-035 & Yes \\
\hline & TRA/ETR & $\begin{array}{l}76.2-\mathrm{m} \text { stack, } 9.4 \mathrm{~m}^{3} / \mathrm{s} \text { discharge capacity, } \\
\text { continuously monitored. As of March } 1988 \text {, this } \\
\text { stack is not monitored. No activity is discharged. }\end{array}$ & TRA-753-001 & Yes \\
\hline & TRA/Hot Cells & $\begin{array}{l}15.2-\mathrm{m} \text { stack, } 1.6 \mathrm{~m}^{3} / \mathrm{s} \text { discharge capacity, } \\
\text { continuously monitored. Three stacks sampled as } \\
\text { one. }\end{array}$ & TRA-632-030 & Yes \\
\hline & TRA/MTR & $\begin{array}{l}\text { 76.2-m stack, } 6 \mathrm{~m}^{3} / \mathrm{s} \text { discharge capacity, } \\
\text { continuously monitored. }\end{array}$ & TRA-710-001 & Yes \\
\hline & TRA/661 & $\begin{array}{l}\text { 8.43-m stack, } 3.3 \mathrm{~m}^{3} / \mathrm{s} \text { discharge capacity, not } \\
\text { monitored. }\end{array}$ & TRA-661-008 & Yes \\
\hline & WERF/North & $\begin{array}{l}15 \text {-m stack, } 8 \mathrm{~m}^{3} / \mathrm{s} \text { discharge capacity, } \\
\text { continuously monitored. }\end{array}$ & PER-755-001 & Yes \\
\hline & WERF/South & $\begin{array}{l}15-\mathrm{m} \text { stack, } 9.4 \mathrm{~m}^{3} / \mathrm{s} \text { discharge capacity, } \\
\text { continuously monitored. }\end{array}$ & PER-756-001 & Yes \\
\hline & WERF $/ 65$ & $\begin{array}{l}15.5-\mathrm{m} \text { stack, } 4.7 \mathrm{~m}^{3} / \mathrm{s} \text { discharge capacity, } \\
\text { continuously monitored. }\end{array}$ & PER-765-001 & Yes \\
\hline & WMF/SWEPP & $\begin{array}{l}4.5-\mathrm{m} \text { stack, } 1.7 \mathrm{~m}^{3} / \mathrm{s} \text { discharge capacity, } \\
\text { continuously monitored during active Drum Vent } \\
\text { operation. }\end{array}$ & WMF-615-001 & Yes \\
\hline $\begin{array}{l}\text { Liquid } \\
\text { (injection } \\
\text { well) }\end{array}$ & INTEC & $\begin{array}{l}181 \mathrm{~m} \text { deep ( } 42.7 \mathrm{~m} \text { below water table), constantly } \\
\text { monitored by radiation detector with a detection } \\
\text { unit of } 2 \times 10^{-6} \mathrm{Ci} / \mathrm{mL} \text {, flow approximately } 170 \mathrm{x} \\
10^{6} \text { liters per month. (INEL discharges to the } \\
\text { injection well have been terminated since } 1986 . \\
\text { This well was closed in November } 1989 .)\end{array}$ & & No \\
\hline & PBF & $\begin{array}{l}33.5 \mathrm{~m} \text { deep ( } 105 \mathrm{~m} \text { above water table), constantly } \\
\text { monitored, flow intermittent with a maximum of } \\
750 \text { liters per minute (no longer in use as of } \\
\text { August } 1980 \text {; in June } 1984 \text {, this well was } \\
\text { completely filled with concrete). }\end{array}$ & & No \\
\hline
\end{tabular}


Liquid

(seepage

ponds,

leaching

pits, cribs)
ARA

CF

INTEC/Pond No. 1

INTEC/Pond No. 2

CTF (formerly LOF)

NRF

TAN

TRA

TRA

Solid
Surface depression (approximately 1.3 acre), No estimate flow $1.05 \times 10^{7}$ liters per year, continuously monitored. ARA completely shut down operations in September 1986, no further releases are being made to this release point except for drainage as a result of rain water runoff.

Sewage plant tile drain field, $610 \times 61 \mathrm{~m}$, average flow approximately $15 \times 10^{6}$ liters per month, continuously monitored.

Percolation pond, $412 \times 480 \times 16 \mathrm{ft}$. CPP 797 is the discharge point that is continuously monitored and sampled.

Percolation pond, $498 \times 498 \times 12$ to $14 \mathrm{ft}$. CPP 797 is the discharge point that is continuously monitored and sampled.

Continuously monitored pond approximately $76 \times 152 \times$ maximum $5.5 \mathrm{~m}$ deep.

Continuously monitored leaching beds handling 95,000 liters per month (releases ended April 1979).

Continuously monitored pond approximately 13 hectares in size. Two ponds $40 \times 73 \mathrm{~m}$ and one pond $76 \times 122 \mathrm{~m}$, average flow approximately $90 \times 10^{6}$ liters per month, prior to 1988 . In 1988, average flow decreased to $4.7 \times 10^{6}$ liters per month. Ponds are continuously monitored. Removed from service August 1993.

Evaporation pond. Two adjacent lined ponds measuring $70 \times 140 \mathrm{~m}$ each. Double liner and leak detection system. All particulate activity remains in lined pond $3 \times 10^{6}$ liters per month.

Continuously monitored. Zero release to soil column.

Disposal of radioactive solid waste accomplished at the INEEL Radioactive Waste Management Facility (WMF).

Storage of transuranic radioactive solid waste accomplished at the INEEL WMF.

Reduction of applicable radioactive solid waste volume accomplished at the INEEL WERF.
N/A Yes

N/A

Yes

Yes

Yes

Yes Yes No Yes

N/A Yes

N/A Yes 